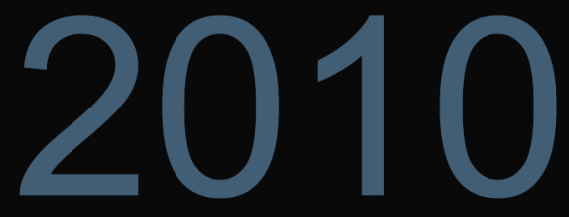

\title{
Idaho National Laboratory Annual IIIness and Injury Surveillance Report
}

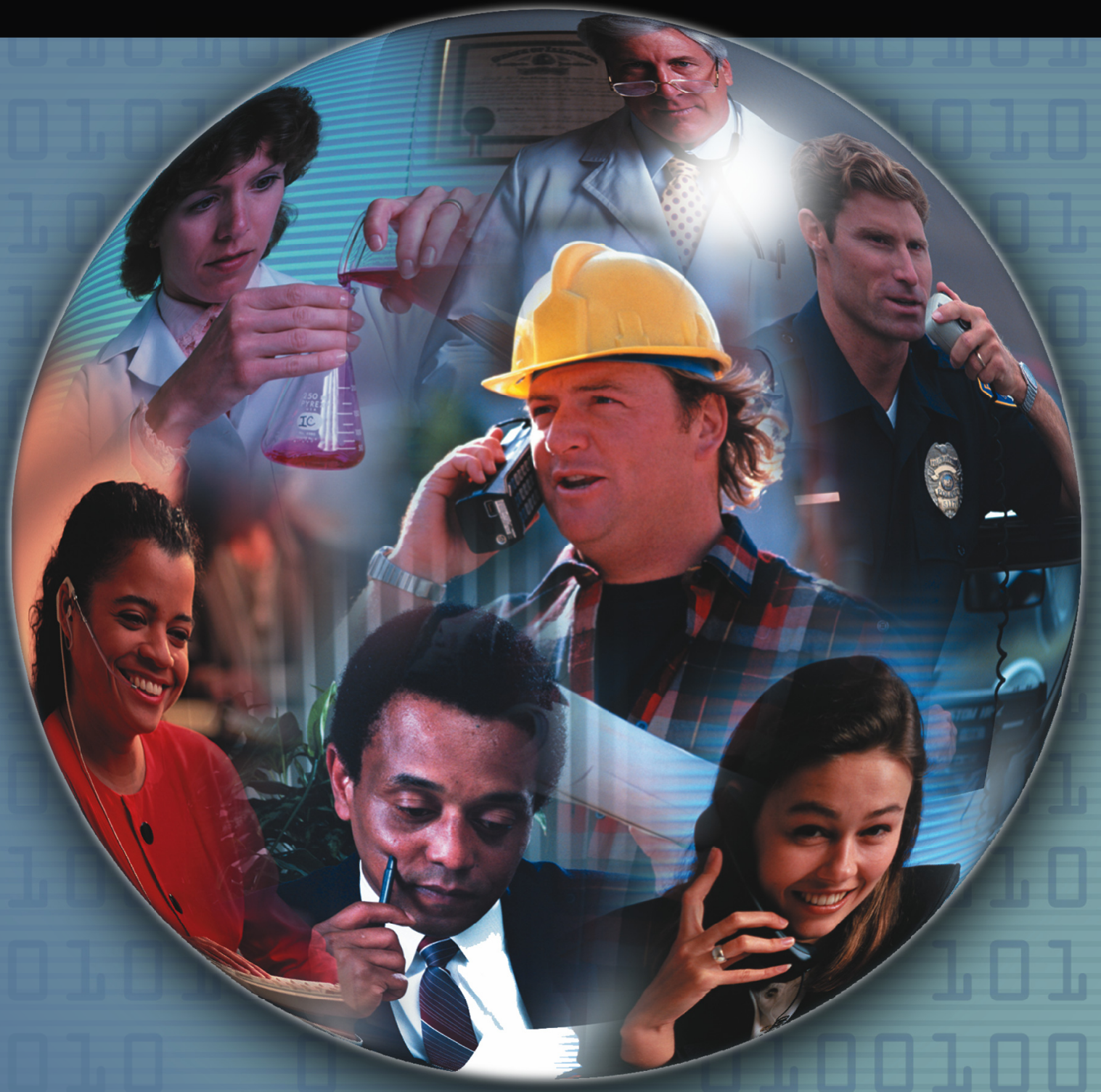




\section{Idaho National Laboratory 2010 Illness and Injury Surveillance Report}

Questions or comments about this report or the Illness and Injury Surveillance Program (IISP) may be directed to:

E-mail:

Dr. Cliff Strader at cliff.strader@hq.doe.gov or Dr. Bonnie Richter at bonnie.richter@hq.doe.gov

or direct letters to:

Mail Stop HS-13 / GTN Building

U.S. Department of Energy

1000 Independence Avenue, S.W.

Washington, DC 20585-0270

Additional information about the Department of Energy's Office of Illness and Injury Prevention Programs, the IISP, and annual reports for DOE sites participating in this program can be found at:

http://www.hss.energy.gov/healthsafety/WSHP/epi/surv/

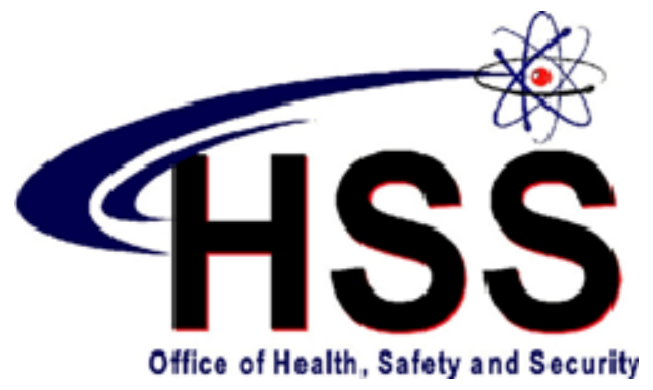

ACKNOWLEDGEMENT

LifeART images copyright 2000 Lippincott Williams \& Wilkins. All rights reserved.

This document was produced under contract number DE-AC05-06OR23100 between the

U.S. Department of Energy and Oak Ridge Associated Universities. 


\section{Idaho National Laboratory \\ 2010 Illness and Injury Surveillance Report}

At A Glance

NOTE: In previous years, the number of days absent or lost and the age of the work force were reported as averages. Beginning in 2010, they are being reported as medians. A median is the value in a population that half the values exceed and half fall short of it. The median is less affected than the average when the distribution contains a few very large or very small values and provides a more representative summary measure.

There were 5,052 people employed at INL in 2010, less than a 1 percent decrease from 2009. The INL work force has steadily declined since 2006 when 6,756 workers were on the roster. Women comprised one-fourth of the work force at INL, as they have since 2006.

Forty-nine percent of workers were 50 years of age or older. The median age for women in 2010 was 48 years. For men, the median age was 50 years. The median age of the INL work force has increased 2 years for both men and women since 2006 .

The absence rate among all workers in 2010 was 9 absences per 100 workers, compared with 12 absences per 100 workers in 2009. This rate decrease was not due to a decrease in any particular diagnosis group. The absence rate in women was similar to the rate for men (10 absences per 100 women compared with 9 absences per 100 men). This similarity is contrary to the absence rates among the DOE sites participating in IISP from 1999 to 2008, with women typically reporting higher absence rates than men.

Injuries, musculoskeletal conditions, and digestive disorders were the top 3 diagnosis categories reported by both men and women at INL in 2010, accounting for 66 percent and 70 percent of diagnoses, respectively.

We noted 5 possible occupational sentinel health events (SHEOs) reported in 2010, all diagnoses for carpal tunnel syndrome. The SHEOs were reported by 
5 workers; they were all aged 40 years or older and were from 4 occupational groups. These diagnoses were responsible for a total of 141 days absent.

Discrepancies between the number of 2009 OSHA events reported by CAIRS in 2009 and 2010 suggest that underreporting was present in the 2009 CAIRS data. For this reason, numbers in the 2010 report's At-A-Glance that refer to 2009 OSHA events do not match the number of OSHA events in the Appendices of the 2009 report. The discussion that follows reflects comparisons made with the corrected 2009 OSHA data.

The total of 56 OSHA events reported in 2010 has remained constant since 2008. Women reported 17 OSHA events, and men reported 39 OSHA events. A total of 131 diagnoses were reported for OSHA events in 2010, with 50 percent the result of injuries. All of the OSHA events were the result of accidents, 66 percent of which involved falls and overexertion and strenuous movements.

\begin{abstract}
A significant change in age-adjusted rates for OSHA-recordable events occurred among Line Operators and female Crafts workers. Although the number of events in 2009 and 2010 were the same, the distribution of events among occupational categories changed significantly. Among women, 11 of the 17 events in 2010 were among Crafts workers and Line Operators, whereas in 2009 , only 1 of 14 events was reported by a female Crafts worker and no events were reported by female Line Operators. Among men, 10 of the 39 events in 2010 were reported by Line Operators compared with only 1 of 42 events in 2009.
\end{abstract}
Although the total number of lost or restricted workdays increased only slightly from 1710 days in 2009 to 1790 days in 2010, women had a six-fold increase in days lost and restricted (69 to 481 days), while the total number of days among men decreased 20 percent (1641 to 1309 days). 
The Idaho National Laboratory

Work Force - 2010

The Work Force by Gender and Age

The Work Force by Gender and Job

Category

\section{Number and Length of Absences}

Absence Rate by Gender and Age 2

Number of Days Absent by

Gender and Age

Absence Rate by Job Category

and Gender .. 3

Median Duration of Absence by

Job Category and Gender. .. 3

\section{Diagnostic Categories}

Number of Diagnoses and Lost Calendar Days by Diagnostic Category

(Categorized by ICD-9-CM) and Gender

Common Diagnoses Among Female

Workers in 2010 .

Common Diagnoses Among Male

Workers in 2010 ... 6

Number of Most Frequently Reported Diagnoses by Job Category and Gender 7

\section{Rates of Disease Occurrence}

Rates for All Illnesses and Injuries Combined by Job Category, Gender, and Age

Rates for Selected Diagnostic Categories by Job Category, Gender, and Age . .8

\section{Time Trends}

Age-Adjusted Rates for All Diagnoses Combined Among Women and Men from 2006 to 2010 10
Age-Adjusted Rates for Selected Diagnostic Categories Among Women and Men from 2006 to 2010

Age-Adjusted Rates for All Diagnoses

Combined Among Women and Men by Job

Category from 2006 to $2010 \ldots \ldots \ldots \ldots \ldots \ldots \ldots \ldots . \ldots 12$

\section{Sentinel Health Events for Occupations (SHEOs)}

Characteristics of SHEOs by Gender 13

SHEO Diagnoses by Gender 13

\section{Occupational Safety and Health Administration (OSHA)-Recordable Events}

OSHA-Recordable Events by Gender and Age. 14

OSHA-Recordable Events by Job

Category and Gender 14

\section{Diagnostic and Accident Categories for OSHA-Recordable Events}

OSHA-Recordable Diagnoses by

Diagnostic Category and Gender

OSHA-Recordable Accidents by Type

and Gender

\section{Rates of OSHA-Recordable Events}

OSHA-Recordable Rates by Age and Job

Categories Among Women, All Diagnoses

Combined

OSHA-Recordable Rates by Age and Job

Categories Among Men, All Diagnoses

Combined

Time Trends for OSHA-Recordable Events

Age-Adjusted Rates for All OSHA-Recordable Diagnoses Combined Among Women and Men by Job Category from 2006 to 2010 .

\section{Appendices}

Appendices A-W .20 


\section{The Idaho National Laboratory Work Force - 2010}

Figure 1. The Work Force by Gender and Age

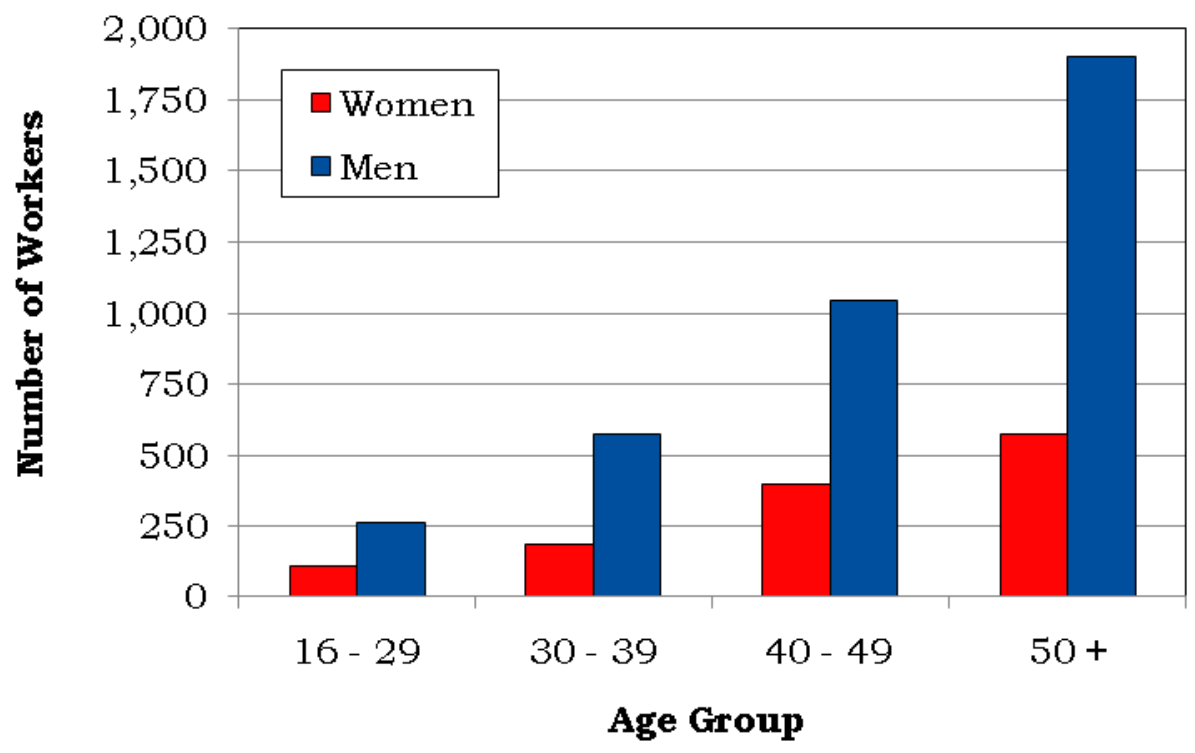

Figure 2. The Work Force by Gender and Job Category

\begin{tabular}{|l|c|c|}
\hline \multicolumn{1}{|c|}{ Job Category } & Women & Men \\
\hline \multirow{2}{*}{ Professional } & 323 & 1,462 \\
& $25 \%$ & $39 \%$ \\
\hline \multirow{2}{*}{ Administrative Support } & 634 & $\mathbf{7 1 1}$ \\
& $50 \%$ & $19 \%$ \\
\hline \multirow{2}{*}{ Technical Support } & 142 & $\mathbf{5 4 1}$ \\
& $11 \%$ & $14 \%$ \\
\hline \multirow{2}{*}{ Service } & $\mathbf{7 1}$ & $\mathbf{2 4 9}$ \\
& $6 \%$ & $\mathbf{7 \%}$ \\
\hline \multirow{2}{*}{ Security and Fire } & 37 & 208 \\
& $3 \%$ & $\mathbf{5 \%}$ \\
\hline \multirow{2}{*}{ Crafts } & 37 & 491 \\
& $3 \%$ & $13 \%$ \\
\hline \multirow{2}{*}{ Line Operators } & 21 & 125 \\
& $2 \%$ & $3 \%$ \\
\hline \multirow{2}{*}{ Total } & 1,265 & $\mathbf{3 , 7 8 7}$ \\
& $100 \%$ & $100 \%$ \\
\hline
\end{tabular}




\section{Number and Length of Absences}

Figure 3. Absence Rate by Gender and Age

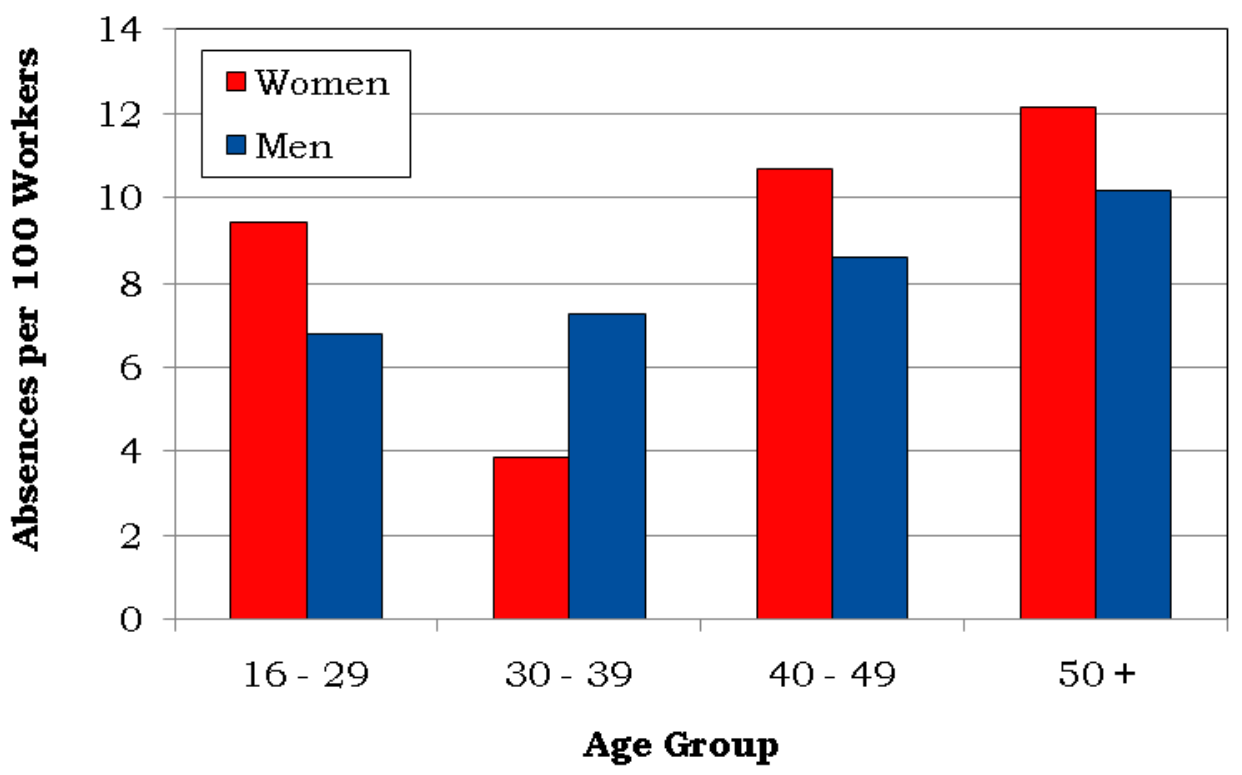

Figure 4. Number of Days Absent by Gender and Age

\begin{tabular}{|c|c|c|c|c|}
\hline \multirow{3}{*}{ Gender } & \multirow{2}{*}{ Age } & \multirow{2}{*}{$\begin{array}{c}\text { Number of } \\
\text { Absences }\end{array}$} & \multicolumn{2}{|c|}{ Number of Days Absent } \\
\cline { 3 - 5 } & & 10 & Total & M edian \\
\hline \multirow{4}{*}{ Women* } & $16-29$ & 7 & 230 & 10 \\
\cline { 2 - 5 } & $30-39$ & 43 & 1,790 & 21 \\
\cline { 2 - 5 } & $40-49$ & 70 & 2,763 & 27 \\
\cline { 2 - 5 } & $50+$ & 130 & 5,090 & 25 \\
\cline { 2 - 5 } & Total & 18 & 855 & 15 \\
\hline \multirow{4}{*}{ Men } & $16-29$ & 42 & 1,497 & 25 \\
\cline { 2 - 5 } & $30-39$ & 90 & 5,697 & 21 \\
\cline { 2 - 5 } & $40-49$ & 193 & 8,285 & 28 \\
\cline { 2 - 5 } & $50+$ & 343 & 16,334 & 26 \\
\cline { 2 - 5 } & Total & & & \\
\cline { 2 - 5 } & & & & \\
\hline
\end{tabular}

*Normal pregnancies were excluded from absences for women. 
Figure 5. Absence Rate by Job Category and Gender

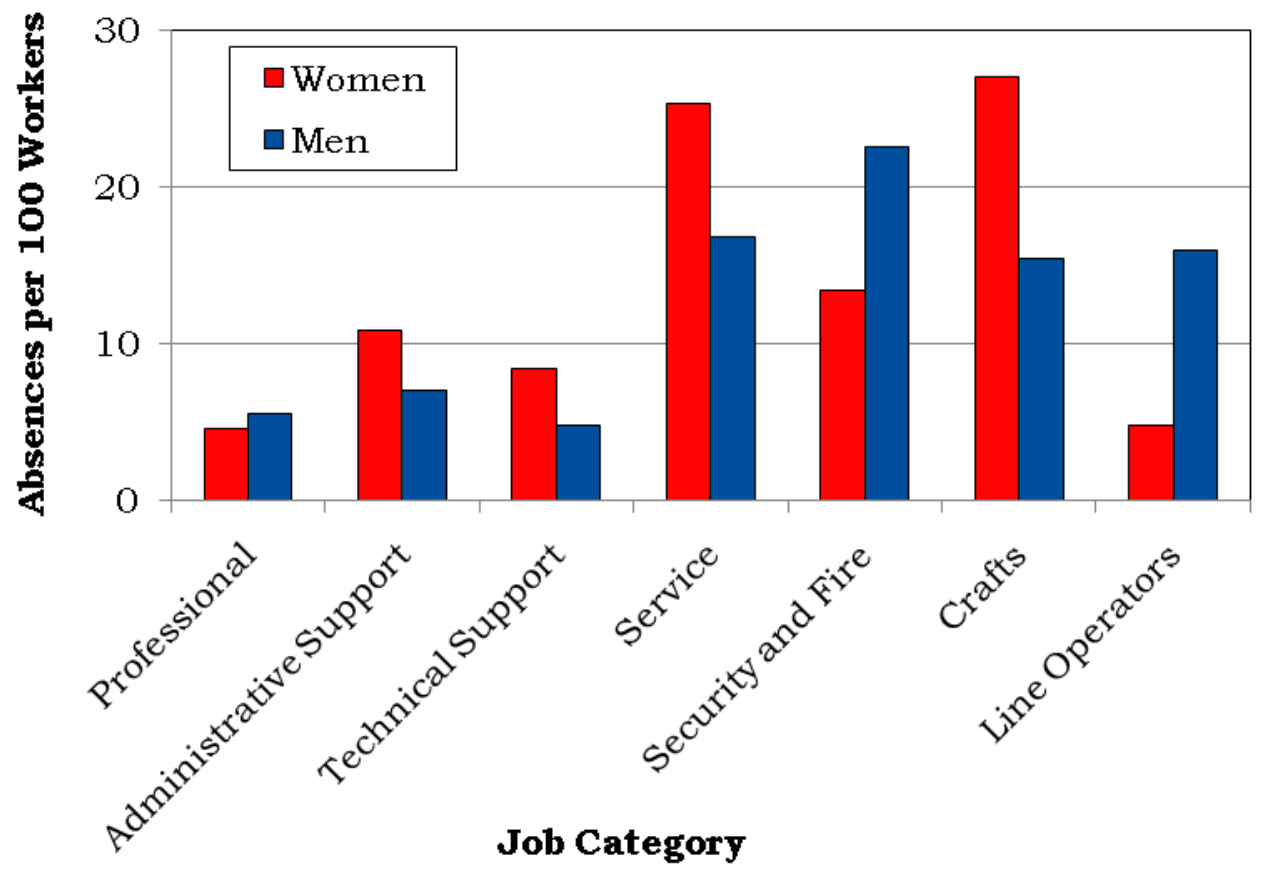

Figure 6. Median Duration of Absence by Job Category and Gender

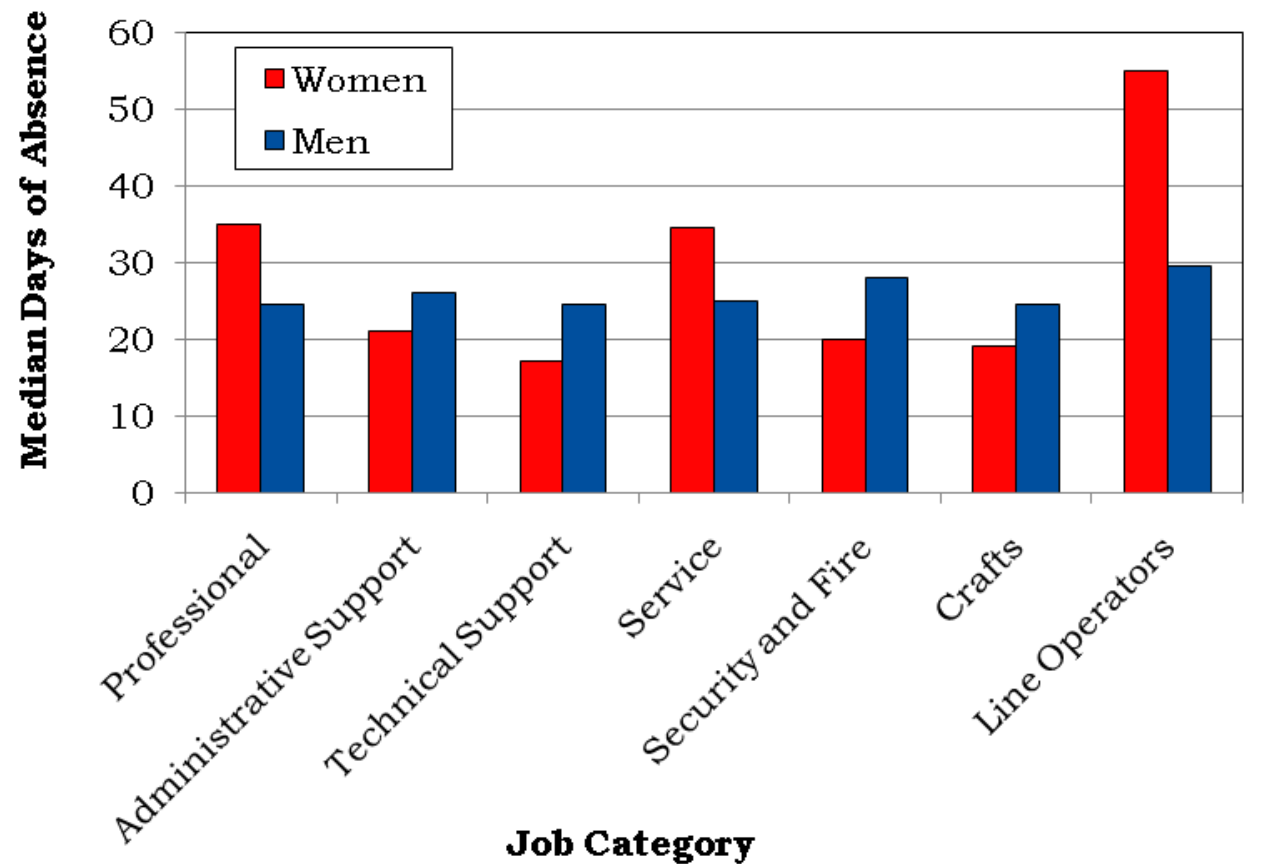




\section{Diagnostic Categories}

Figure 7. Number of Diagnoses and Lost Calendar Days by Diagnostic Category (Categorized by ICD-9-CM) and Gender

\begin{tabular}{|c|c|c|c|c|}
\hline \multirow[b]{2}{*}{ Diagnostic Category } & \multicolumn{2}{|c|}{ Women } & \multicolumn{2}{|c|}{ Men } \\
\hline & $\begin{array}{l}\text { Number of } \\
\text { Diagnoses }\end{array}$ & $\begin{array}{c}\text { Number } \\
\text { of Lost } \\
\text { Calendar } \\
\text { Days }\end{array}$ & $\begin{array}{l}\text { Number of } \\
\text { Diagnoses }\end{array}$ & $\begin{array}{c}\text { Number } \\
\text { of Lost } \\
\text { Calendar } \\
\text { Days }\end{array}$ \\
\hline Benign Growths & 2 & 239 & 1 & 20 \\
\hline Blood & 0 & 0 & 0 & $\mathbf{0}$ \\
\hline Cancer & $\mathbf{0}$ & 0 & 10 & 870 \\
\hline Digestive & 16 & 253 & 45 & 2,105 \\
\hline Endocrine / Me tabolic & 1 & 32 & 8 & 110 \\
\hline Existing Birth Condition & 0 & 0 & $\mathbf{0}$ & $\mathbf{0}$ \\
\hline Genitourinary & 12 & 257 & 8 & 252 \\
\hline Heart/Circulatory & 4 & 62 & 32 & 651 \\
\hline Infections/Parasites & 4 & 139 & 2 & 46 \\
\hline Injury & 60 & 2,107 & 138 & 6,198 \\
\hline Miscarriage & 0 & 0 & NA & NA \\
\hline Musculoskeletal & 48 & 2,293 & 89 & 5,113 \\
\hline Nervous System & 4 & 100 & 22 & 722 \\
\hline Psychological & 3 & 85 & 2 & 10 \\
\hline Respiratory & 13 & 218 & 41 & 828 \\
\hline Skin & 0 & 0 & 2 & 50 \\
\hline Unspecified Symptoms & 9 & 47 & 13 & 144 \\
\hline
\end{tabular}

Note: Lost calendar days for each absence are counted more than once when multiple diagnoses occur in different diagnostic categories for the same absence. 


\section{Figure 8. Common Diagnoses Among Female Workers in 2010}

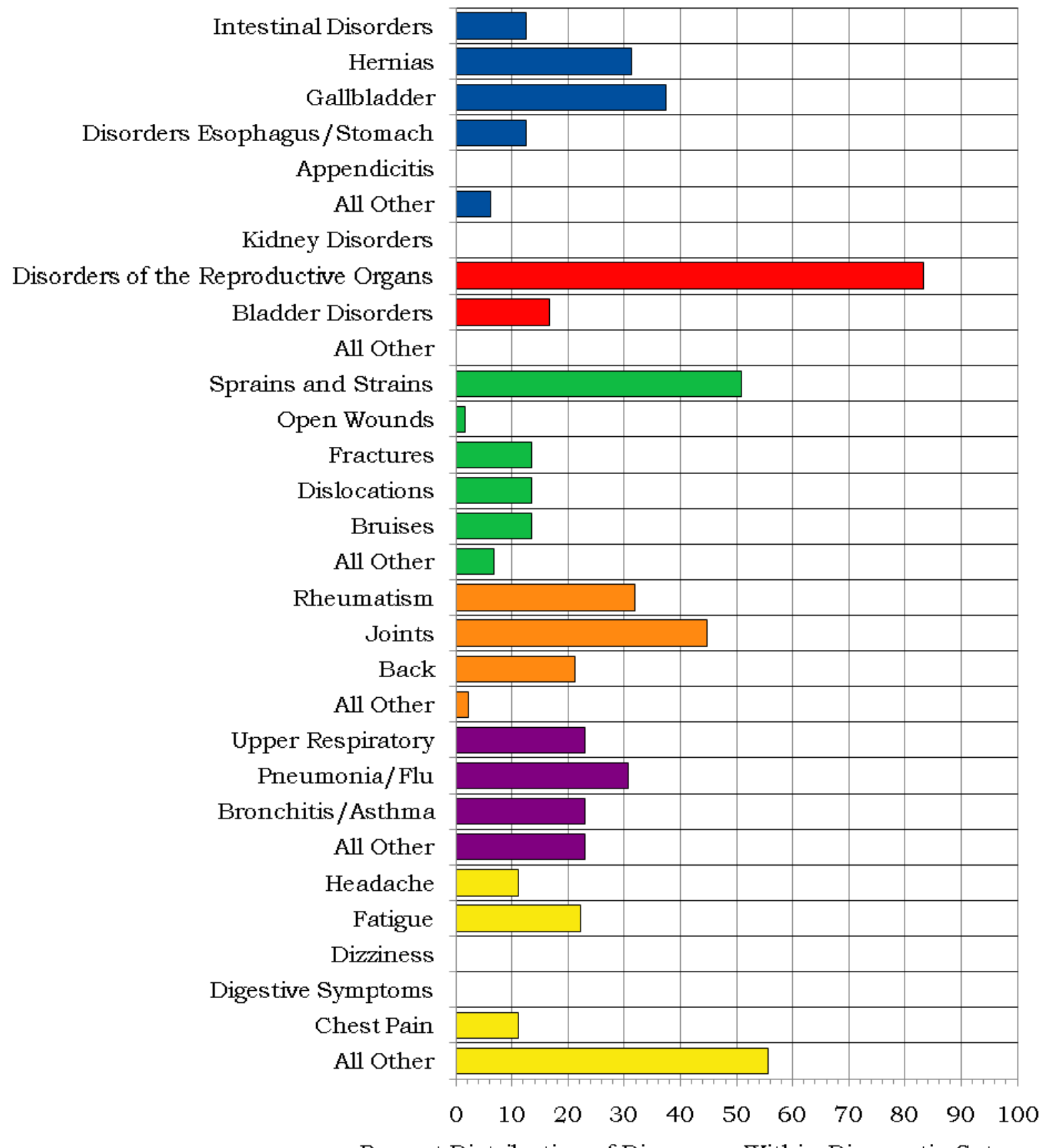

Percent Distribution of Diagnoses Within Diagnostic Category

Digestive, 16 Diagnoses

Genitourinary, 12 Diagnoses

Injury, 59 Diagnoses
Musculoskeletal, 47 Diagnoses

Respiratory, 13 Diagnoses

Unspecified Symptoms, 9 Diagnoses 


\section{Figure 9. Common Diagnoses Among Male Workers in 2010}

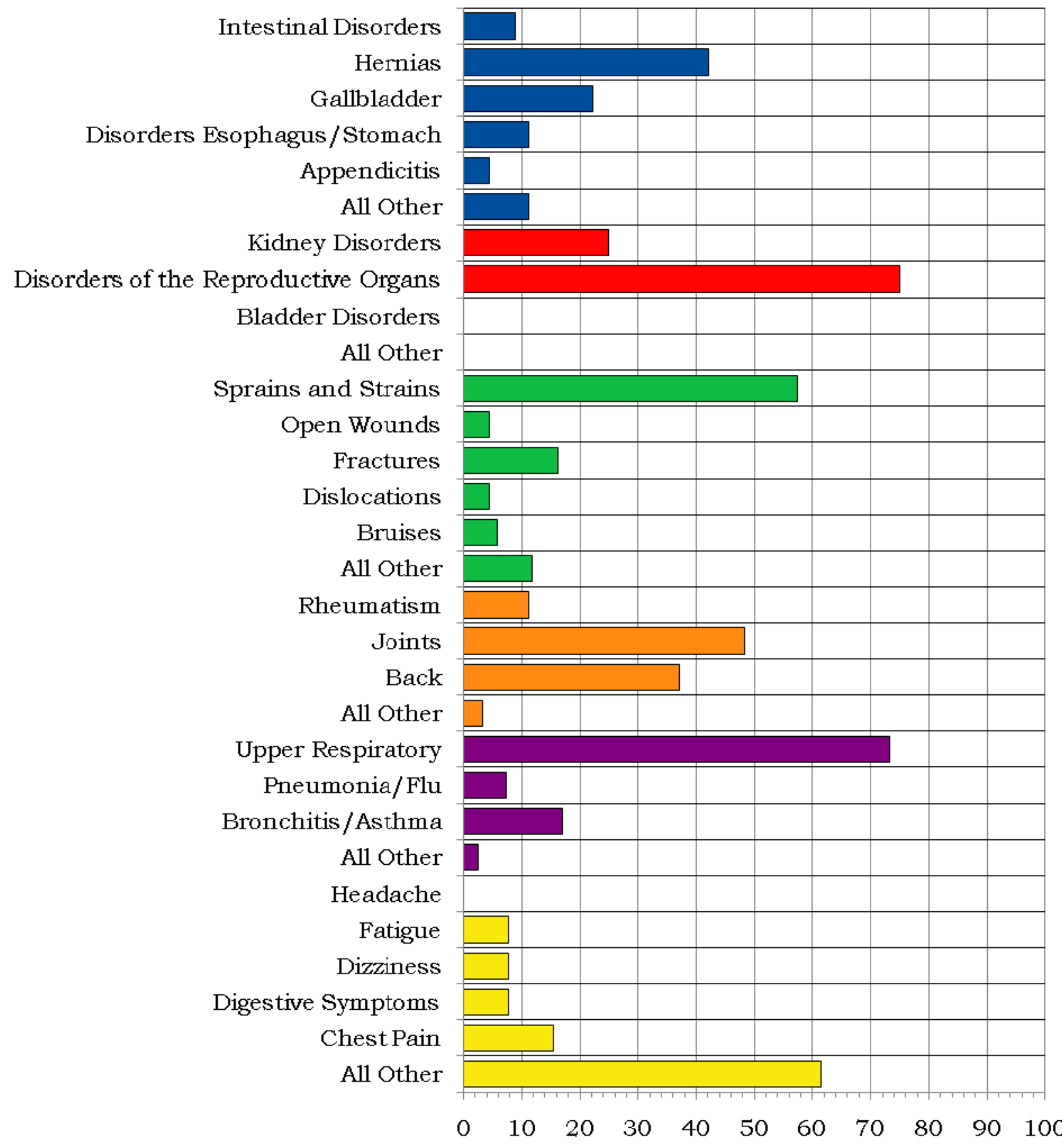

Percent Distribution of Diagnoses Within Diagnostic Category

Digestive, 45 Diagnoses

Genitourinary, 8 Diagnoses

Injury, 136 Diagnoses
Musculoskeletal, 89 Diagnoses

Respiratory, 41 Diagnoses

Unspecified Symptoms, 13 Diagnoses 


\section{Figure 10. Number of Most Frequently Reported Diagnoses by Job Category and Gender}

\begin{tabular}{|c|c|c|c|c|}
\hline Job Category & Men & & Women & \\
\hline \multirow{4}{*}{ Professional } & Injury & 28 & M usculoskeletal & 6 \\
\hline & M usculoskeletal & 26 & Injury & 6 \\
\hline & Digestive & 15 & Respiratory & 2 \\
\hline & & & Unspecified Symptoms & 2 \\
\hline \multirow{4}{*}{ Administrative Support } & Injury & 19 & Injury & 35 \\
\hline & Digestive & 9 & M usculoskeletal & 27 \\
\hline & M usculoskeletal & 7 & Digestive & 9 \\
\hline & Respiratory & 7 & & \\
\hline \multirow{4}{*}{ Technical Support } & Injury & 12 & Respiratory & 4 \\
\hline & M usculoskeletal & 6 & Injury & 4 \\
\hline & Digestive & 3 & Unspecified Symptoms & 3 \\
\hline & Nervous System & 3 & & \\
\hline \multirow{3}{*}{ Service } & Injury & 18 & Injury & 9 \\
\hline & M usculoskeletal & 17 & M usculoskeletal & 8 \\
\hline & Heart/Circulatory & 7 & Digestive & 2 \\
\hline \multirow{4}{*}{ Security and Fire } & Injury & 23 & M usculoskeletal & 4 \\
\hline & M usculoskeletal & 11 & Respiratory & 1 \\
\hline & Respiratory & 8 & Genitourinary & 1 \\
\hline & & & Heart/Circulatory & 1 \\
\hline \multirow{4}{*}{ Crafts } & Injury & 30 & Injury & 6 \\
\hline & M usculoskeletal & 14 & M usculoskeletal & 2 \\
\hline & Respiratory & 14 & Respiratory & 2 \\
\hline & & & Digestive & 2 \\
\hline \multirow{3}{*}{ Line Operators } & Injury & 8 & M usculoskeletal & 1 \\
\hline & M usculoskeletal & 8 & & \\
\hline & Nervous System & 2 & & \\
\hline
\end{tabular}




\section{Rates of Disease Occurrence}

Figure 11. Rates for All Illnesses and Injuries Combined by Job Category, Gender, and Age

\begin{tabular}{|c|c|c|c|c|}
\hline \multirow{2}{*}{$\begin{array}{c}\text { All Illnesses \& } \\
\text { Injuries Combined }\end{array}$} & \multicolumn{4}{|c|}{ Rate per 1,000} \\
\hline & Job Category & Age & Men & Women \\
\hline & \multirow{2}{*}{ Professional } & $<50$ & 64 & 52 \\
\hline & & $50+$ & 75 & 70 \\
\hline & \multirow{2}{*}{ Administrative Support } & $<50$ & 72 & 129 \\
\hline & & $50+$ & 93 & 175 \\
\hline & \multirow{2}{*}{ Technical Support } & $<50$ & 44 & 115 \\
\hline & & $50+$ & 64 & 109 \\
\hline & \multirow{2}{*}{ Service } & $<50$ & 177 & 208 \\
\hline & & $50+$ & 242 & 340 \\
\hline & \multirow{2}{*}{ Security and Fire } & $<50$ & 256 & 353 \\
\hline & & $50+$ & 253 & 50 \\
\hline & \multirow{2}{*}{ Crafts } & $<50$ & 178 & 240 \\
\hline & & $50+$ & 185 & 667 \\
\hline & \multirow{2}{*}{ Line Operators } & $<50$ & 148 & 0 \\
\hline & & $50+$ & 211 & 77 \\
\hline
\end{tabular}

Figure 12. Rates for Selected Diagnostic Categories by Job Category, Gender, and Age

\begin{tabular}{|c|c|c|c|c|}
\hline \multirow{2}{*}{ Cancer } & \multicolumn{4}{|c|}{ Rate per 1,000} \\
\hline & Job Category & Age & Men & Women \\
\hline & Profe scional & $<50$ & 1 & $\mathbf{0}$ \\
\hline & & $50+$ & 4 & 0 \\
\hline & Administrative Sunmort & $<50$ & 6 & 0 \\
\hline & & $50+$ & 3 & 0 \\
\hline & Technical Support & $<50$ & 0 & 0 \\
\hline & 1 ecrintical support & $50+$ & 0 & 0 \\
\hline & Service & $<50$ & 0 & 0 \\
\hline & Service & $50+$ & 7 & 0 \\
\hline & & $<50$ & 0 & 0 \\
\hline & securicy and Fire & $50+$ & 0 & 0 \\
\hline & Crafts & $<50$ & 0 & 0 \\
\hline & Cralts & $50+$ & 9 & 0 \\
\hline & Line Oper & $<50$ & 0 & 0 \\
\hline & & $50+$ & 0 & 0 \\
\hline
\end{tabular}


Figure 12. Rates for Selected Diagnostic Categories by Job Category, Gender, and Age (Continued)

\begin{tabular}{|l|l|c|c|c|}
\hline \multirow{2}{*}{ Heart/Circulatory } & \multicolumn{4}{|c|}{ Rate per 1,000 } \\
\cline { 2 - 5 } & \multicolumn{1}{|c|}{ Job Category } & Age & M en & W omen \\
\cline { 2 - 5 } & \multirow{2}{*}{ Profe ssional } & $<50$ & $\mathbf{4}$ & $\mathbf{0}$ \\
\cline { 2 - 5 } & $50+$ & $\mathbf{1 1}$ & $\mathbf{8}$ \\
\cline { 2 - 5 } & \multirow{2}{*}{ Administrative Support } & $<50$ & $\mathbf{6}$ & $\mathbf{0}$ \\
\cline { 2 - 5 } & $50+$ & $\mathbf{3}$ & $\mathbf{6}$ \\
\cline { 2 - 5 } & \multirow{2}{*}{ Technical Support } & $<50$ & $\mathbf{6}$ & $\mathbf{0}$ \\
\cline { 2 - 5 } & $50+$ & $\mathbf{0}$ & $\mathbf{0}$ \\
\cline { 2 - 5 } & \multirow{2}{*}{ Service } & $<50$ & $\mathbf{1 0}$ & $\mathbf{0}$ \\
\cline { 2 - 5 } & $50+$ & $\mathbf{3 9}$ & $\mathbf{0}$ \\
\cline { 2 - 5 } & \multirow{2}{*}{ Security and Fire } & $<50$ & $\mathbf{8}$ & $\mathbf{5 9}$ \\
\cline { 2 - 5 } & $50+$ & $\mathbf{1 1}$ & $\mathbf{0}$ \\
\cline { 2 - 5 } & \multirow{2}{*}{ Crafts } & $<50$ & $\mathbf{8}$ & $\mathbf{0}$ \\
\cline { 2 - 5 } & $50+$ & $\mathbf{9}$ & $\mathbf{0}$ \\
\cline { 2 - 5 } & \multirow{2}{*}{ Line Operators } & $<50$ & $\mathbf{0}$ & $\mathbf{0}$ \\
\cline { 2 - 5 } & $50+$ & $\mathbf{1 4}$ & $\mathbf{0}$ \\
\hline
\end{tabular}

\begin{tabular}{|l|l|c|c|c|}
\hline \multirow{2}{*}{ Respiratory } & \multicolumn{4}{|c|}{ Rate per 1,000 } \\
\cline { 2 - 5 } & \multicolumn{1}{|c|}{ Job Category } & Age & M en & W omen \\
\hline \multirow{2}{*}{ Professional } & $<50$ & $\mathbf{6}$ & $\mathbf{5}$ \\
\cline { 2 - 5 } & $50+$ & $\mathbf{5}$ & $\mathbf{8}$ \\
\hline \multirow{2}{*}{ Administrative Support } & $<50$ & $\mathbf{6}$ & $\mathbf{3}$ \\
\cline { 2 - 5 } & $50+$ & $\mathbf{1 4}$ & $\mathbf{6}$ \\
\hline \multirow{2}{*}{ Technical Support } & $<50$ & $\mathbf{0}$ & $\mathbf{2 1}$ \\
\cline { 2 - 5 } & $50+$ & $\mathbf{1 0}$ & $\mathbf{4 3}$ \\
\hline \multirow{2}{*}{ Service } & $<50$ & $\mathbf{1 0}$ & $\mathbf{0}$ \\
\cline { 2 - 5 } & $50+$ & $\mathbf{0}$ & $\mathbf{2 1}$ \\
\hline \multirow{2}{*}{ Security and Fire } & $<50$ & $\mathbf{4 1}$ & $\mathbf{5 9}$ \\
\cline { 2 - 5 } & $50+$ & $\mathbf{3 4}$ & $\mathbf{0}$ \\
\hline \multirow{2}{*}{ Crafts } & $<50$ & $\mathbf{2 7}$ & $\mathbf{0}$ \\
\cline { 2 - 5 } & $50+$ & $\mathbf{3 0}$ & $\mathbf{1 6 7}$ \\
\cline { 2 - 5 } & \multirow{2}{*}{ Line Operators } & $<50$ & $\mathbf{1 9}$ & $\mathbf{0}$ \\
\cline { 2 - 5 } & $50+$ & $\mathbf{0}$ & $\mathbf{0}$ \\
\hline
\end{tabular}


Figure 12. Rates for Selected Diagnostic Categories by Job Category, Gender, and Age (Continued)

\begin{tabular}{|c|c|c|c|c|}
\hline \multirow{2}{*}{ Injury } & \multicolumn{4}{|c|}{ Rate per 1,000} \\
\hline & Job Category & Age & Men & Women \\
\hline & \multirow{2}{*}{ Professional } & $<50$ & 25 & 21 \\
\hline & & $50+$ & 11 & 16 \\
\hline & \multirow{2}{*}{ Administrative Support } & $<50$ & 14 & 68 \\
\hline & & $50+$ & 38 & 42 \\
\hline & \multirow{2}{*}{ Technical Support } & $<50$ & 27 & 0 \\
\hline & & $50+$ & 15 & 65 \\
\hline & \multirow{2}{*}{ Service } & $<50$ & 63 & 167 \\
\hline & & $50+$ & 78 & 106 \\
\hline & \multirow{2}{*}{ Security and Fire } & $<50$ & 107 & 0 \\
\hline & & $50+$ & 115 & 0 \\
\hline & \multirow{2}{*}{ Crafts } & $<50$ & 69 & 200 \\
\hline & & $50+$ & 52 & 83 \\
\hline & \multirow{2}{*}{ Line Operators } & $<50$ & 56 & 0 \\
\hline & & $50+$ & 70 & 0 \\
\hline
\end{tabular}

Time Trends

Figure 13. Age-Adjusted Rates for All Diagnoses Combined Among Women and Men from 2006 to 2010*

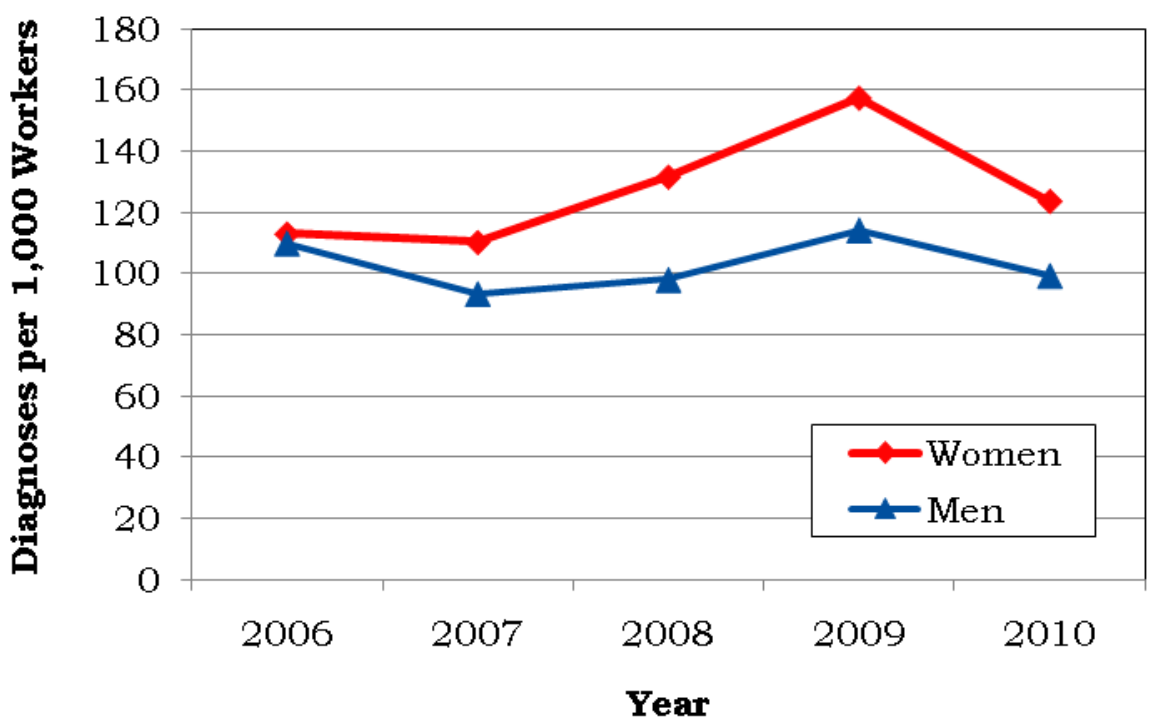

*Standardized to age distribution of 2000 U.S. population. 
Figure 14. Age-Adjusted Rates for Selected Diagnostic Categories Among Women and Men from 2006 to 2010*

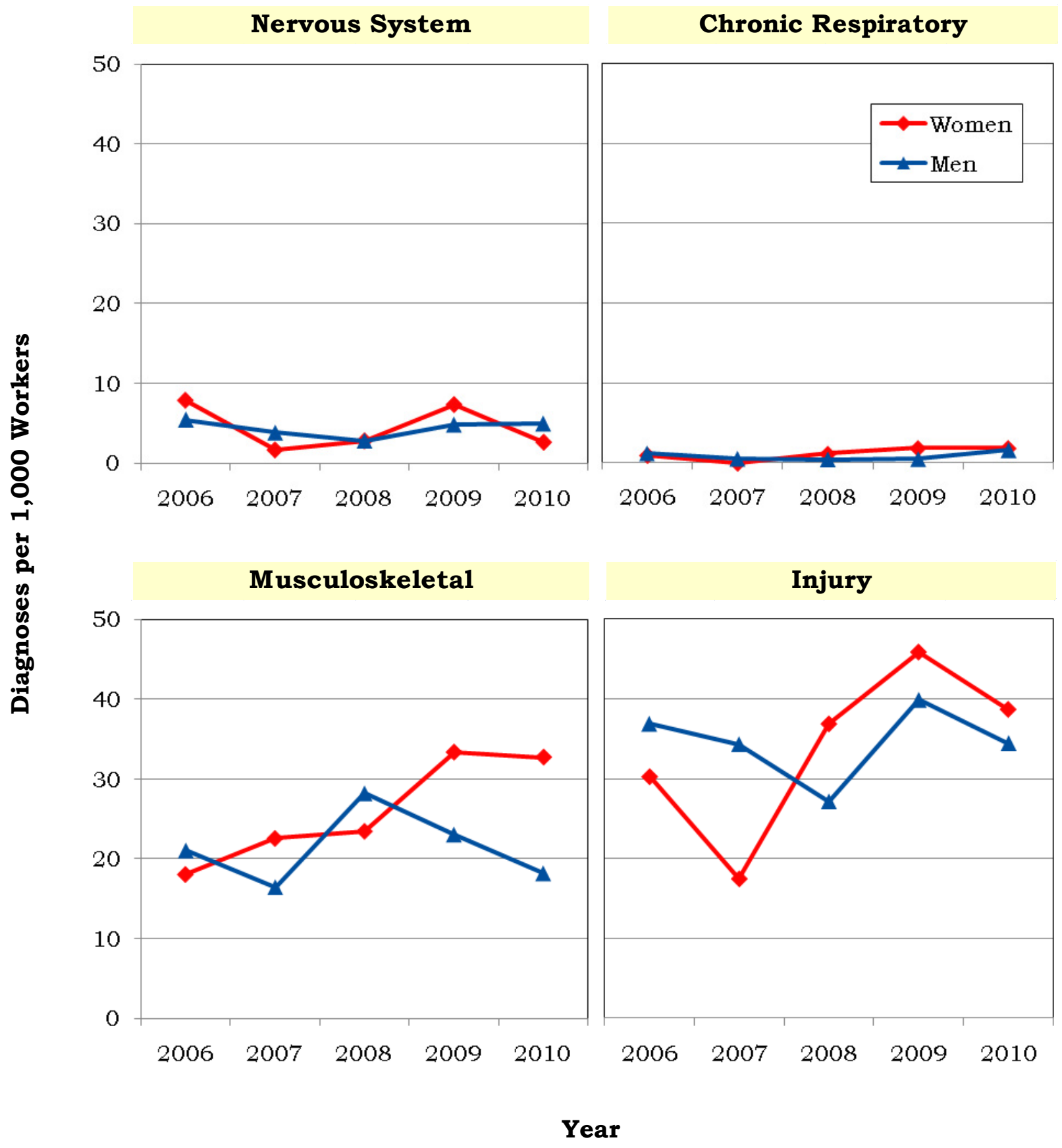

*Standardized to age distribution of 2000 U.S. population. 
Figure 15. Age-Adjusted Rates for All Diagnoses Combined Among Women and Men by Job Category from 2006 to 2010*

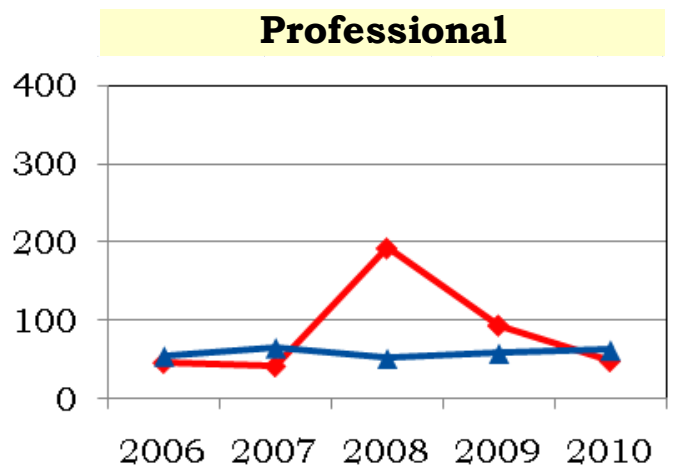

$\leadsto$ Women

$\rightarrow$ Men

Administrative Support
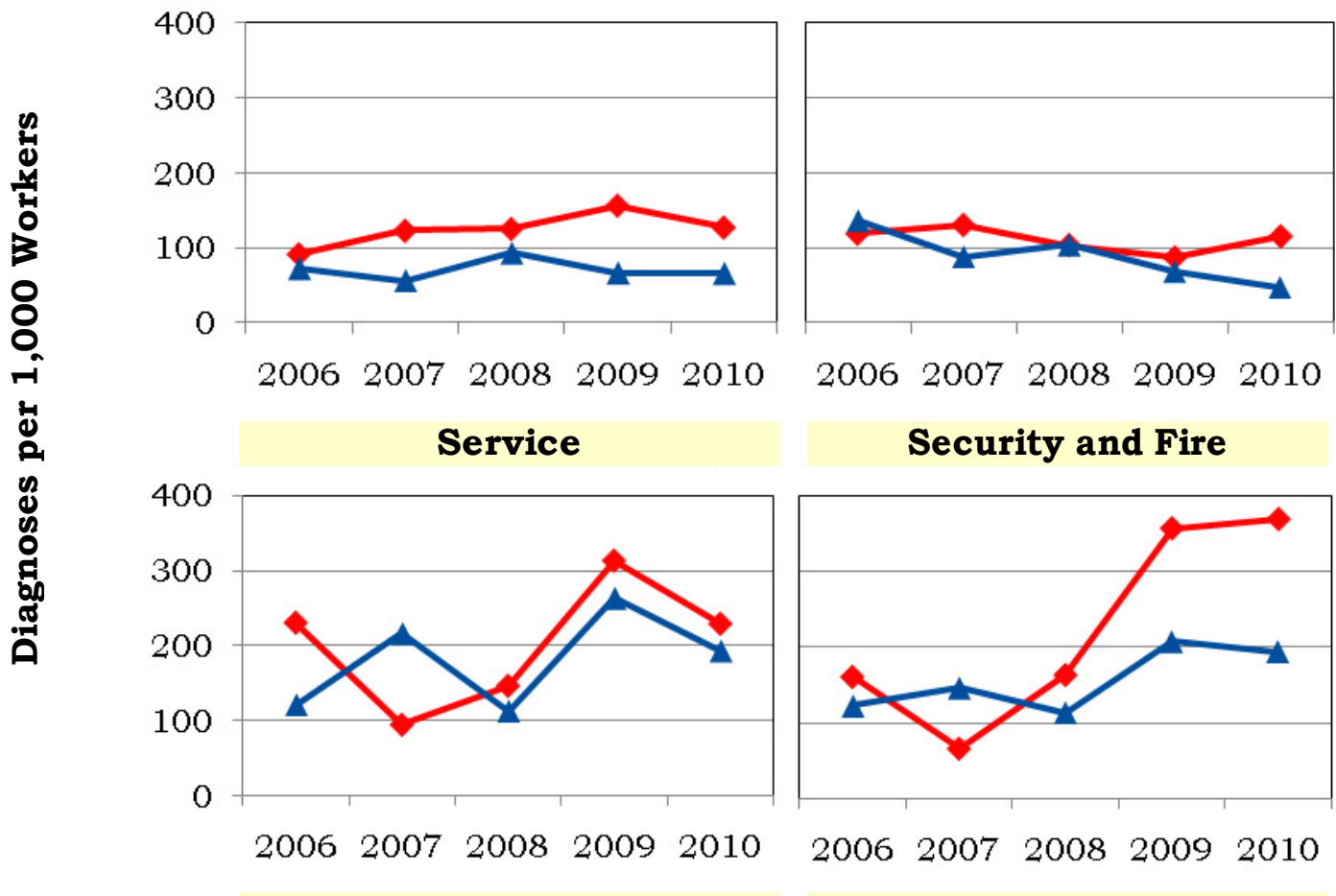

Security and Fire

ญ

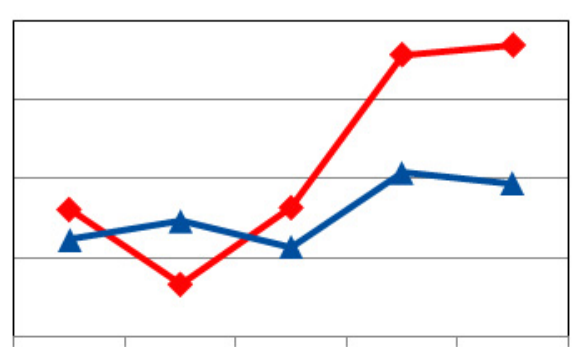

Crafts

20062007200820092010

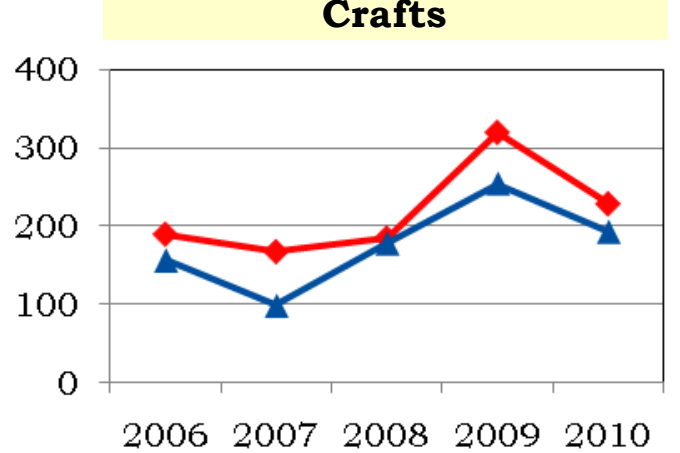

Line Operators

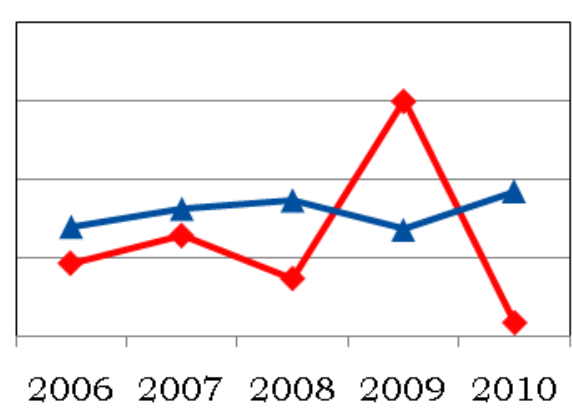

Year

*Standardized to age distribution of 2000 U.S. population. 


\section{Sentinel Health Events for Occupations (SHEOs)}

An occupational sentinel health event (SHEO) is a disease, disability, or death that is likely to be occupationally related. Although sentinel health events may indicate an occupational exposure, many may result from nonoccupational exposures. Sentinel health events are therefore assessed in two categories:

Definite Sentinel Health Events: Diseases that are unlikely to occur in the absence of an occupational exposure (e.g., asbestosis).

Possible Sentinel Health Events: Diseases that may be occupational but can also occur in the absence of an occupational exposure (e.g., lung cancer or carpal tunnel syndrome).

Figure 16. Characteristics of SHEOs by Gender

\begin{tabular}{|l|c|c|c|c|}
\hline \multirow{2}{*}{} & \multicolumn{2}{|c|}{$\begin{array}{c}\text { Total Number of } \\
\text { SHEO Diagnoses }\end{array}$} & \multicolumn{2}{c|}{$\begin{array}{c}\text { Total Number of } \\
\text { Days Absent }\end{array}$} \\
\cline { 2 - 5 } & Men & Women & Men & W omen \\
\hline Definite & 0 & 0 & 0 & 0 \\
\hline Possible & 3 & 2 & 84 & 57 \\
\hline Total & 3 & 2 & 84 & 57 \\
\hline
\end{tabular}

Figure 17. SHEO Diagnoses by Gender

\begin{tabular}{|l|c|c|}
\hline \multirow{2}{*}{ Diagnoses } & \multicolumn{2}{c|}{ Gender } \\
\cline { 2 - 3 } & Women & M en \\
\hline Carpal Tunnel Syndrome & 2 & 3 \\
\hline
\end{tabular}


Occupational Safety and Health Administration (OSHA)-Recordable Events

Figure 18. OSHA-Recordable Events by Gender and Age

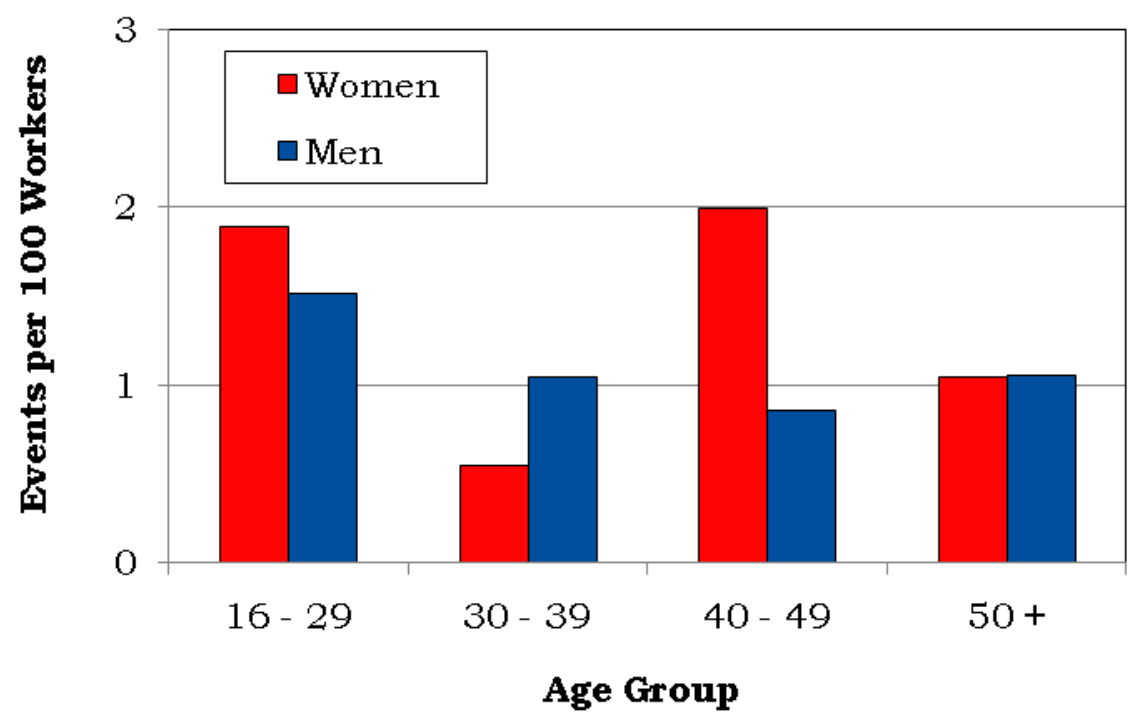

Figure 19. OSHA-Recordable Events by Job Category and Gender

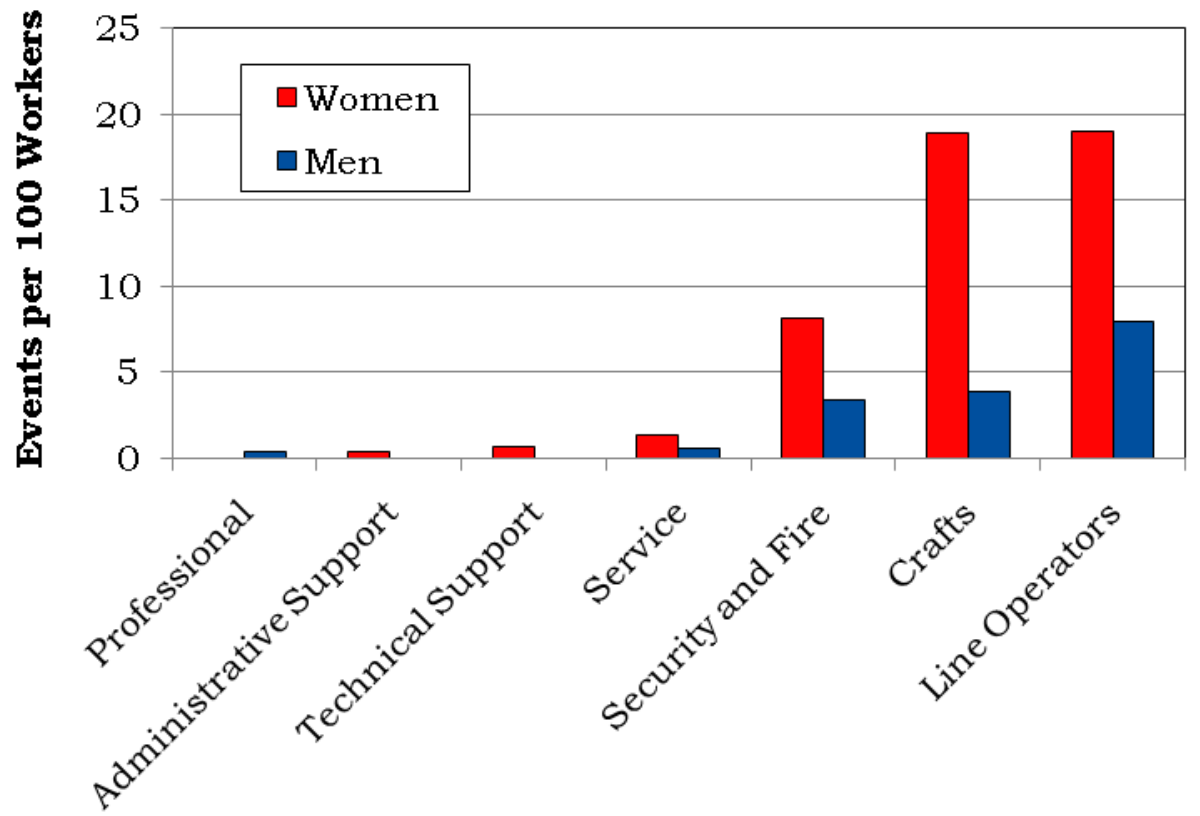

Job Category 


\section{Diagnostic and Accident Categories for OSHA-Recordable Events}

Figure 20. OSHA-Recordable Diagnoses by Diagnostic Category and Gender

\begin{tabular}{|l|c|c|}
\hline \multirow{2}{*}{ Diagnostic Category } & \multicolumn{2}{c|}{ Gender } \\
\cline { 2 - 3 } & Women & M en \\
\hline Digestive & $\mathbf{0}$ & $\mathbf{2}$ \\
\hline Heart/Circulatory & $\mathbf{0}$ & $\mathbf{1}$ \\
\hline Musculoskeletal & $\mathbf{1 8}$ & $\mathbf{3 4}$ \\
\hline Nervous System & $\mathbf{2}$ & $\mathbf{2}$ \\
\hline Unspecified Symptoms & $\mathbf{3}$ & $\mathbf{4}$ \\
\hline Injury & $\mathbf{2 0}$ & $\mathbf{4 5}$ \\
\hline Fractures - Upper Limb & $\mathbf{1}$ & $\mathbf{2}$ \\
\hline Fractures - Lower Limb & $\mathbf{0}$ & $\mathbf{2}$ \\
\hline Dislocations & $\mathbf{0}$ & $\mathbf{4}$ \\
\hline Back Sprains \& Strains & $\mathbf{7}$ & $\mathbf{5}$ \\
\hline Other Sprains \& Strains & $\mathbf{8}$ & $\mathbf{1 3}$ \\
\hline Open Wounds - Head, Neck, Trunk & $\mathbf{1}$ & $\mathbf{2}$ \\
\hline Open Wounds - Upper Limb & $\mathbf{1}$ & $\mathbf{8}$ \\
\hline Open Wounds - Lower Limb & $\mathbf{0}$ & $\mathbf{1}$ \\
\hline Superficial Injuries & $\mathbf{0}$ & $\mathbf{2}$ \\
\hline Bruises & $\mathbf{0}$ & $\mathbf{4}$ \\
\hline Foreign Bodies Entering Orifice & $\mathbf{0}$ & $\mathbf{1}$ \\
\hline Unspecified Injuries & $\mathbf{2}$ & $\mathbf{1}$ \\
\hline
\end{tabular}


Figure 21. OSHA-Recordable Accidents by Type and Gender

\begin{tabular}{|l|c|c|}
\hline \multirow{2}{*}{ Accident Category } & \multicolumn{2}{c|}{ Gender } \\
\cline { 2 - 3 } & Women & M en \\
\cline { 2 - 3 } & $\begin{array}{c}\text { Number of } \\
\text { Accidents }\end{array}$ & $\begin{array}{c}\text { Number of } \\
\text { Accidents }\end{array}$ \\
\hline Motor Vehicle Traffic & $\mathbf{0}$ & $\mathbf{2}$ \\
\hline Motor Vehicle Nontraffic & $\mathbf{0}$ & $\mathbf{1}$ \\
\hline Falls & $\mathbf{2}$ & $\mathbf{9}$ \\
\hline Submersion/Suffocation/Foreign Bodies & $\mathbf{0}$ & $\mathbf{1}$ \\
\hline Other Accidents & $\mathbf{1 5}$ & $\mathbf{2 6}$ \\
\hline Struck by an Object & $\mathbf{0}$ & $\mathbf{6}$ \\
\hline Caught Between Objects & $\mathbf{0}$ & $\mathbf{1}$ \\
\hline Cutting/Piercing Instrument/Object & $\mathbf{1}$ & $\mathbf{4}$ \\
\hline Overexertion/Strenuous Movements & $\mathbf{1 1}$ & $\mathbf{1 5}$ \\
\hline Repetitive Trauma & $\mathbf{3}$ & $\mathbf{0}$ \\
\hline Total & $\mathbf{1 7}$ & $\mathbf{3 9}$ \\
\hline
\end{tabular}




\section{Rates of OSHA-Recordable Events}

Figure 22. OSHA-Recordable Rates by Age and Job Categories Among Women, All Diagnoses Combined

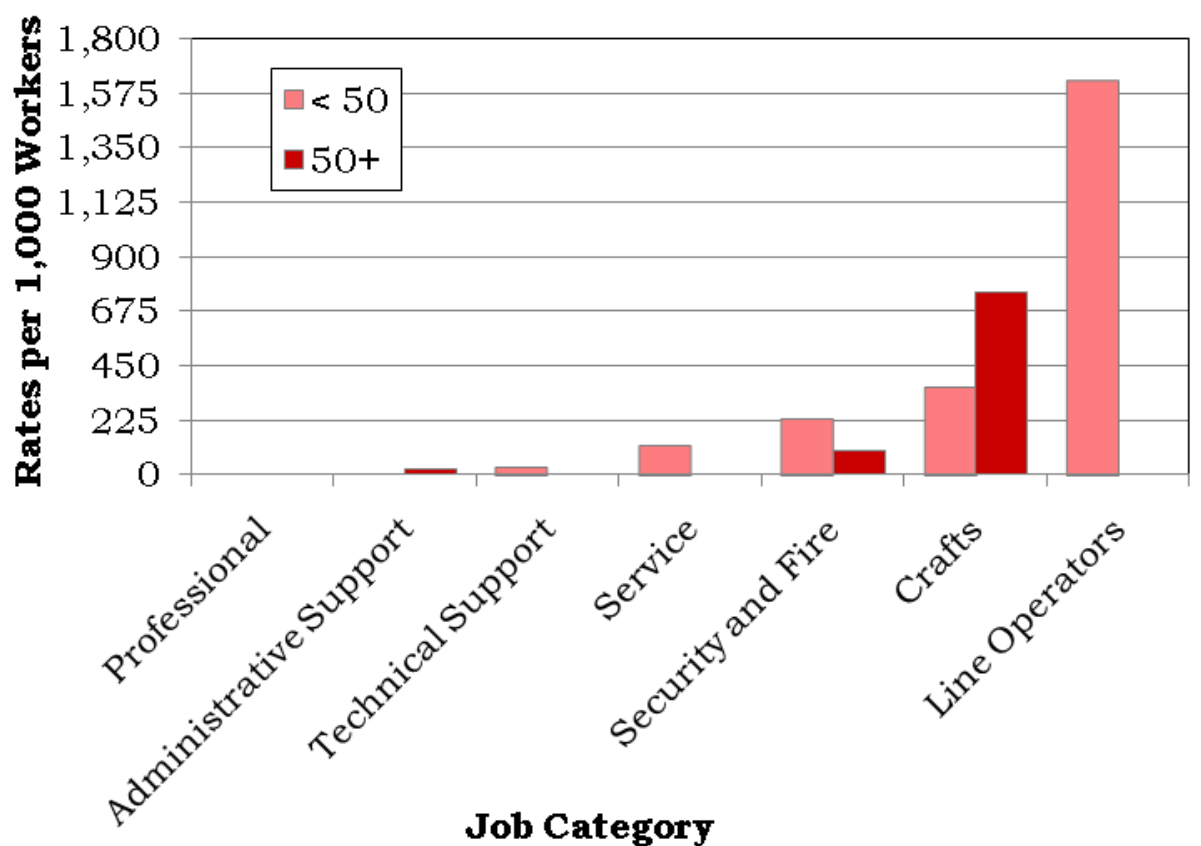

Figure 23. OSHA-Recordable Rates by Age and Job Categories Among Men, All Diagnoses Combined

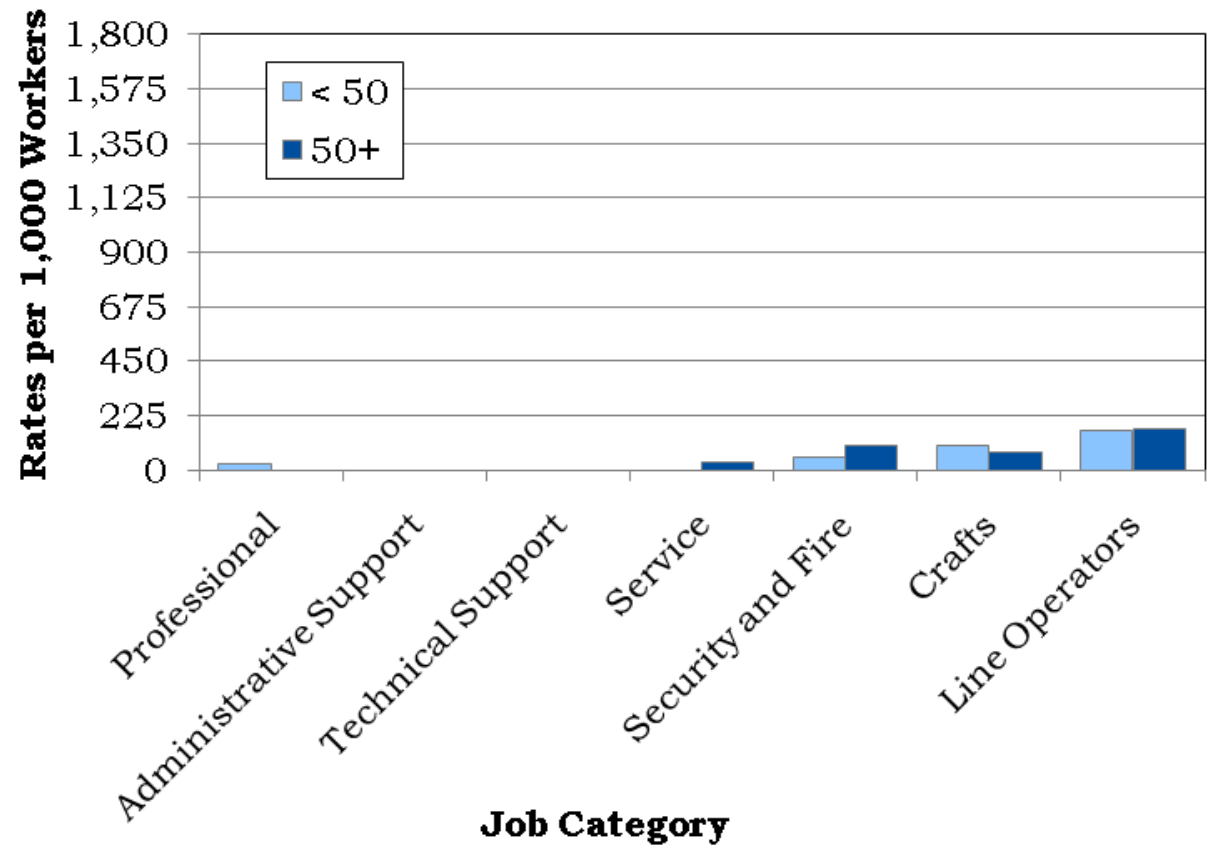




\section{Time Trends for OSHA-Recordable Events}

Figure 24. Age-Adjusted Rates for All OSHA-Recordable Diagnoses Combined Among Women and Men by Job Category from 2006 to 2010*

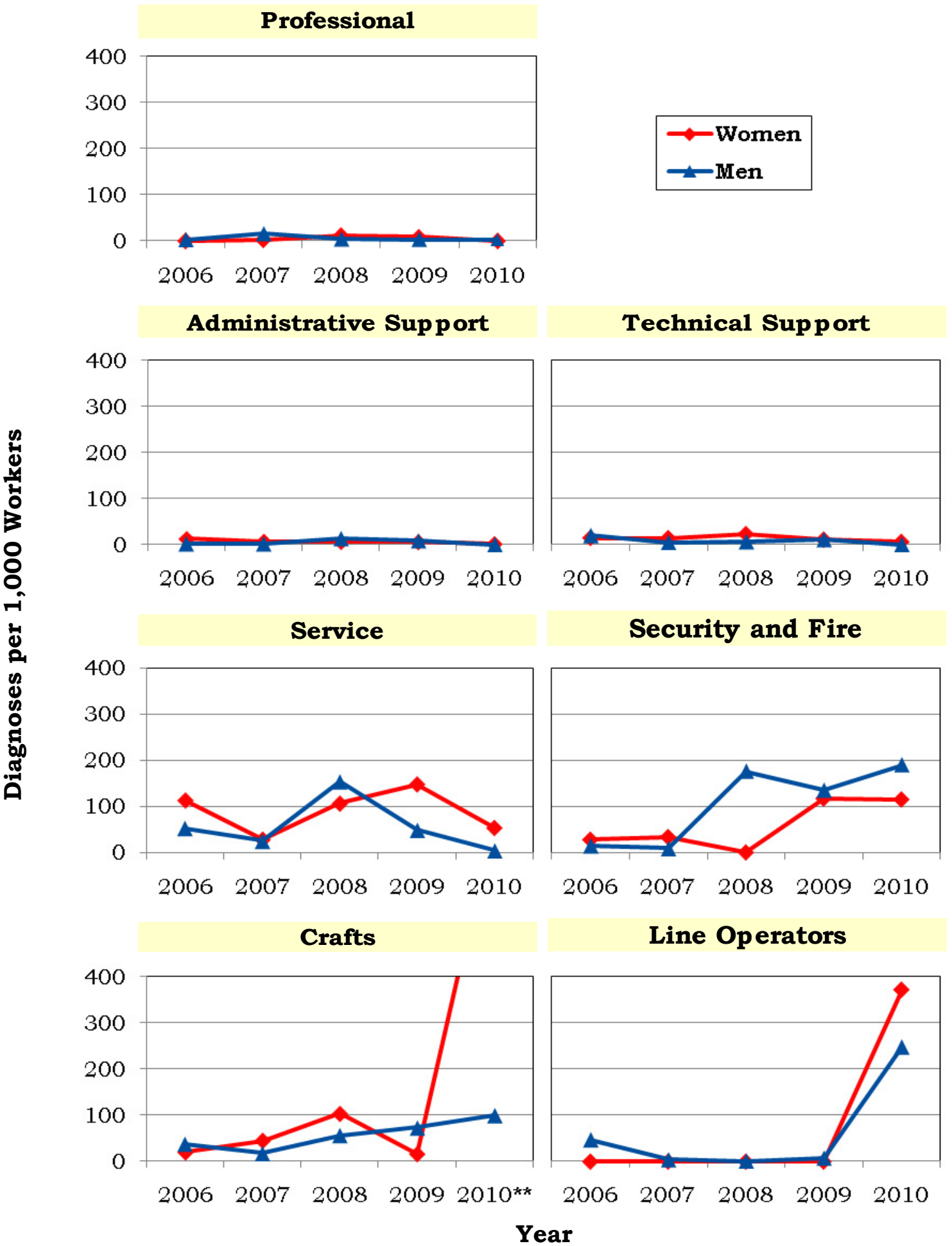

*Standardized to age distribution of 2000 U.S. population.

**The rate for Crafts workers for 2010 was truncated to 400 for graphical presentation. The actual rate was 745.01 diagnoses per 1,000 workers. 


\section{Appendices}


Idaho National Laboratory 2010

Absence Data

Appendix A. Work Force by Gender, Age, and Job Category

\begin{tabular}{|c|c|c|c|c|c|c|c|c|c|c|c|}
\hline \multirow{3}{*}{ Job Category } & \multicolumn{5}{|c|}{ Women } & \multicolumn{5}{|c|}{ Men } & \multirow[b]{3}{*}{ TOTAL } \\
\hline & \multicolumn{4}{|c|}{ Age Group } & \multirow[b]{2}{*}{ TOTAL } & \multicolumn{4}{|c|}{ Age Group } & \multirow[b]{2}{*}{ TOTAL } & \\
\hline & $16-29$ & $30-39$ & $40-49$ & $50+$ & & $16-29$ & $30-39$ & $40-49$ & $50+$ & & \\
\hline Professional & 19 & 46 & 129 & 129 & 323 & 61 & 174 & 436 & 791 & 1,462 & 1,785 \\
\hline Administrative Support & 42 & 99 & 184 & 309 & 634 & 47 & 114 & 186 & 364 & 711 & 1,345 \\
\hline Technical Support & 31 & 21 & 44 & 46 & 142 & 86 & 103 & 150 & 202 & 541 & 683 \\
\hline Service & 5 & 6 & 13 & 47 & 71 & 11 & 35 & 50 & 153 & 249 & 320 \\
\hline Security and Fire & 2 & 5 & 10 & 20 & 37 & 5 & 42 & 74 & 87 & 208 & 245 \\
\hline Crafts & 7 & 1 & 17 & 12 & 37 & 50 & 91 & 118 & 232 & 491 & 528 \\
\hline Line Operators & 0 & 3 & 5 & 13 & 21 & 5 & 17 & 32 & 71 & 125 & 146 \\
\hline TOTAL & 106 & 181 & 402 & 576 & 1,265 & 265 & 576 & 1,046 & 1,900 & 3,787 & 5,052 \\
\hline
\end{tabular}

Appendix B. Age Distribution of the Work Force by Gender

\begin{tabular}{|l|r|r|r|r|r|r|r|r|}
\hline \multirow{3}{*}{ Year } & \multicolumn{9}{|c|}{ Women } & \multicolumn{4}{c|}{ Men } \\
\cline { 2 - 9 } & \multicolumn{1}{|c|}{ Percent Distribution by Age Group } & \multicolumn{1}{c|}{ Percent Distribution by Age Group } \\
\cline { 2 - 10 } & $\mathbf{1 6}-\mathbf{2 9}$ & $\mathbf{3 0}-\mathbf{3 9}$ & $\mathbf{4 0}-\mathbf{4 9}$ & $\mathbf{5 0}+$ & $\mathbf{1 6}-\mathbf{2 9}$ & $\mathbf{3 0}-\mathbf{3 9}$ & $\mathbf{4 0}-\mathbf{4 9}$ & $\mathbf{5 0}+$ \\
\hline $\mathbf{1 9 9 3}$ & 21.01 & 34.35 & 28.99 & 15.65 & 10.30 & 33.87 & 32.67 & 23.17 \\
\hline $\mathbf{1 9 9 4}$ & 22.68 & 33.47 & 27.52 & 16.33 & 10.03 & 30.36 & 32.65 & 26.95 \\
\hline $\mathbf{1 9 9 5}$ & 19.44 & 32.92 & 29.28 & 18.35 & 8.13 & 29.02 & 33.91 & 28.93 \\
\hline $\mathbf{1 9 9 6}$ & 12.46 & 34.28 & 35.33 & 17.93 & 5.75 & 30.58 & 39.53 & 24.14 \\
\hline $\mathbf{1 9 9 7}$ & 10.77 & 32.93 & 36.22 & 20.08 & 5.50 & 27.88 & 39.78 & 26.84 \\
\hline $\mathbf{1 9 9 8}$ & 10.47 & 30.61 & 36.61 & 22.32 & 5.07 & 25.65 & 39.39 & 29.89 \\
\hline $\mathbf{1 9 9 9}$ & 11.02 & 29.59 & 34.58 & 24.81 & 5.68 & 23.14 & 38.01 & 33.18 \\
\hline $\mathbf{2 0 0 0}$ & 10.85 & 28.08 & 35.78 & 25.29 & 5.95 & 22.02 & 37.76 & 34.27 \\
\hline $\mathbf{2 0 0 1}$ & 9.92 & 24.69 & 37.88 & 27.50 & 5.04 & 20.00 & 37.79 & 37.17 \\
\hline $\mathbf{2 0 0 2}$ & 9.18 & 23.21 & 38.52 & 29.10 & 5.57 & 17.85 & 37.32 & 39.27 \\
\hline $\mathbf{2 0 0 3}$ & 7.56 & 21.86 & 40.15 & 30.43 & 6.98 & 16.49 & 38.49 & 38.05 \\
\hline $\mathbf{2 0 0 4}$ & 7.96 & 19.24 & 39.03 & 33.77 & 7.19 & 15.16 & 36.15 & 41.50 \\
\hline $\mathbf{2 0 0 5}$ & 10.55 & 17.03 & 38.37 & 34.05 & 11.18 & 16.18 & 33.05 & 39.58 \\
\hline $\mathbf{2 0 0 6}$ & 8.17 & 16.09 & 37.81 & 37.93 & 7.88 & 14.65 & 33.19 & 44.28 \\
\hline $\mathbf{2 0 0 7}$ & 7.34 & 16.68 & 36.92 & 39.07 & 5.61 & 13.25 & 33.90 & 47.24 \\
\hline $\mathbf{2 0 0 8}$ & 6.04 & 15.67 & 35.86 & 42.43 & 5.62 & 13.32 & 31.44 & 49.62 \\
\hline $\mathbf{2 0 0 9}$ & 7.18 & 14.29 & 34.10 & 44.44 & 6.21 & 14.27 & 29.14 & 50.38 \\
\hline $\mathbf{2 0 1 0}$ & 8.38 & 14.31 & 31.78 & 45.53 & 7.00 & 15.21 & 27.62 & 50.17 \\
\hline
\end{tabular}


Idaho National Laboratory 2010

Absence Data

Appendix C. Total Number of Workers Who Reported at Least One Absence by Gender, Age, and Job Category*

\begin{tabular}{|c|c|c|c|c|c|c|c|c|c|c|c|}
\hline \multirow{3}{*}{ Job Category } & \multicolumn{5}{|c|}{ Women } & \multicolumn{5}{|c|}{ Men } & \multirow[b]{3}{*}{ TOTAL } \\
\hline & \multicolumn{4}{|c|}{ Age Group } & \multirow[b]{2}{*}{ TOTAL } & \multicolumn{4}{|c|}{ Age Group } & \multirow[b]{2}{*}{ TOTAL } & \\
\hline & $16-29$ & 30 - 39 & $40-49$ & $50+$ & & $16-29$ & 30 - 39 & $40-49$ & $50+$ & & \\
\hline Professional & 0 & 1 & 8 & 5 & 14 & 1 & 7 & 21 & 46 & 75 & 89 \\
\hline Administrative Support & 3 & 3 & 23 & 35 & 64 & 2 & 1 & 12 & 28 & 43 & 107 \\
\hline Technical Support & 4 & 1 & 2 & 3 & 10 & 1 & 4 & 7 & 11 & 23 & 33 \\
\hline Service & 0 & 2 & 1 & 12 & 15 & 1 & 4 & 6 & 22 & 33 & 48 \\
\hline Security and Fire & 1 & 0 & 3 & 1 & 5 & 0 & 10 & 15 & 16 & 41 & 46 \\
\hline Crafts & 1 & 0 & 3 & 4 & 8 & 11 & 11 & 13 & 31 & 66 & 74 \\
\hline Line Operators & 0 & 0 & 0 & 1 & 1 & 1 & 3 & 2 & 12 & 18 & 19 \\
\hline TOTAL & 9 & 7 & 40 & 61 & 117 & 17 & 40 & 76 & 166 & 299 & 416 \\
\hline
\end{tabular}

*Only those job categories and gender/age combinations with at least one absence appear in this table.

Appendix D. Total Number of Absences by Gender, Age, and Job Category*

\begin{tabular}{|c|c|c|c|c|c|c|c|c|c|c|c|}
\hline \multirow{3}{*}{ Job Category } & \multicolumn{5}{|c|}{ Women } & \multicolumn{5}{|c|}{ Men } & \multirow[b]{3}{*}{ TOTAL } \\
\hline & \multicolumn{4}{|c|}{ Age Group } & \multirow[b]{2}{*}{ TOTAL } & \multicolumn{4}{|c|}{ Age Group } & \multirow[b]{2}{*}{ TOTAL } & \\
\hline & \begin{tabular}{|l|}
$16-29$ \\
\end{tabular} & 30 - 39 & $40-49$ & $50+$ & & $16-29$ & 30 - 39 & $40-49$ & $50+$ & & \\
\hline Professional & 0 & 1 & 8 & 6 & 15 & 1 & 7 & 23 & 51 & 82 & 97 \\
\hline Administrative Support & 3 & 3 & 25 & 38 & 69 & 2 & 2 & 14 & 32 & 50 & 119 \\
\hline Technical Support & 5 & 1 & 2 & 4 & 12 & 1 & 4 & 8 & 13 & 26 & 38 \\
\hline Service & 0 & 2 & 2 & 14 & 18 & 2 & 4 & 7 & 29 & 42 & 60 \\
\hline Security and Fire & 1 & 0 & 3 & 1 & 5 & 0 & 10 & 18 & 19 & 47 & 52 \\
\hline Crafts & 1 & 0 & 3 & 6 & 10 & 11 & 12 & 18 & 35 & 76 & 86 \\
\hline Line Operators & 0 & $\overline{0}$ & 0 & 1 & 1 & 1 & 3 & 2 & 14 & 20 & 21 \\
\hline TOTAL & 10 & 7 & 43 & 70 & 130 & 18 & 42 & 90 & 193 & 343 & 473 \\
\hline
\end{tabular}

*Only those job categories and gender/age combinations with at least one absence appear in this table. 
Idaho National Laboratory 2010

Absence Data

Appendix E. Distribution of the Number of Calendar Days Missed per Absence by Gender and Age*

\begin{tabular}{|c|c|c|c|c|c|c|c|c|c|c|c|}
\hline \multirow{3}{*}{$\begin{array}{c}\text { Number of } \\
\text { Calendar Days }\end{array}$} & \multicolumn{5}{|c|}{ Women } & \multicolumn{5}{|c|}{ Men } & \multirow[b]{3}{*}{ TOTAL } \\
\hline & \multicolumn{4}{|c|}{ Age Group } & \multirow[b]{2}{*}{ TOTAL } & \multicolumn{4}{|c|}{ Age Group } & \multirow[b]{2}{*}{ TOTAL } & \\
\hline & $16-29$ & $30-39$ & $40-49$ & $50+$ & & $16-29$ & 30 - 39 & $40-49$ & $50+$ & & \\
\hline$<15$ & 6 & 1 & 16 & 17 & 40 & 9 & 17 & 29 & 53 & 108 & 148 \\
\hline $15-28$ & 1 & 4 & 8 & 25 & 38 & 2 & 7 & 25 & 46 & 80 & 118 \\
\hline $29-42$ & 1 & 0 & 7 & 8 & 16 & 2 & 7 & 8 & 33 & 50 & 66 \\
\hline $43-56$ & 0 & 0 & 6 & 7 & 13 & 1 & 3 & 8 & 24 & 36 & 49 \\
\hline $57-91$ & 2 & 0 & 4 & 8 & 14 & 1 & 5 & 8 & 14 & 28 & 42 \\
\hline $92-182$ & 0 & 2 & 1 & 4 & 7 & 2 & 2 & 8 & 18 & 30 & 37 \\
\hline $183+$ & 0 & 0 & 1 & 1 & 2 & 1 & 1 & 4 & 5 & 11 & 13 \\
\hline TOTAL & 10 & 7 & 43 & 70 & 130 & 18 & 42 & 90 & 193 & 343 & 473 \\
\hline
\end{tabular}

*Only those gender/age combinations with at least one absence appear in this table. 
Idaho National Laboratory 2010

Absence Data

Appendix F. Distribution of the Number of Calendar Days Missed per Absence by Gender and Job Category*

Women

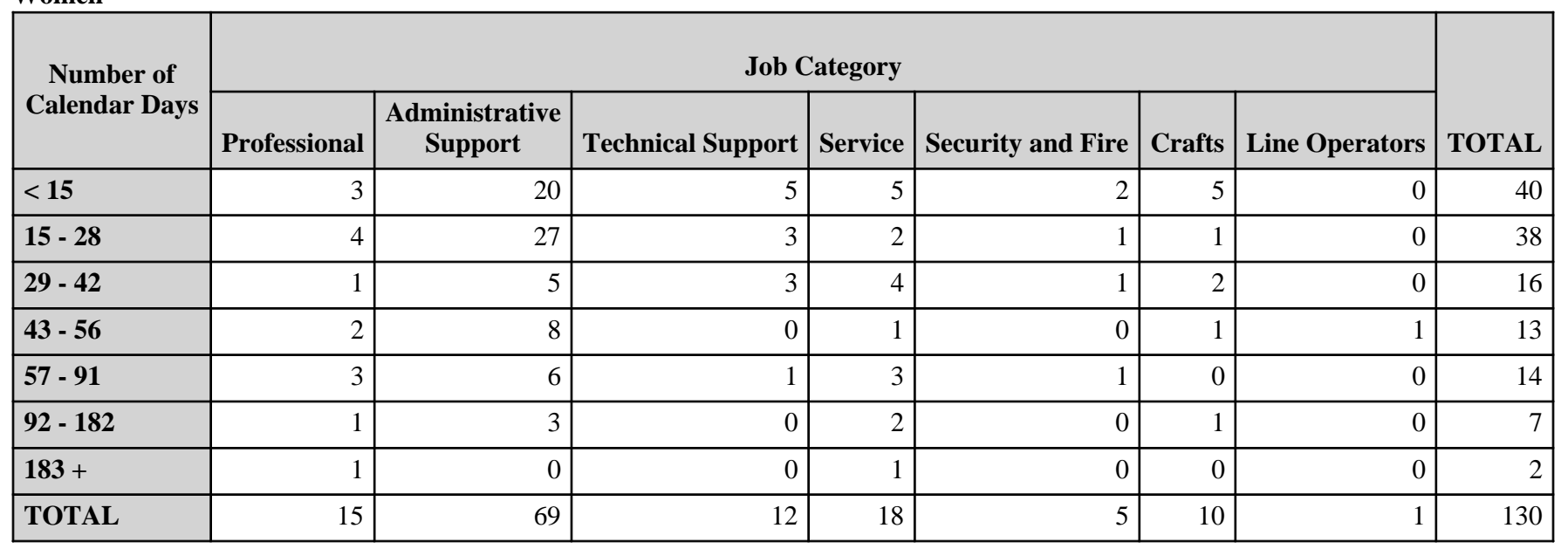

Men

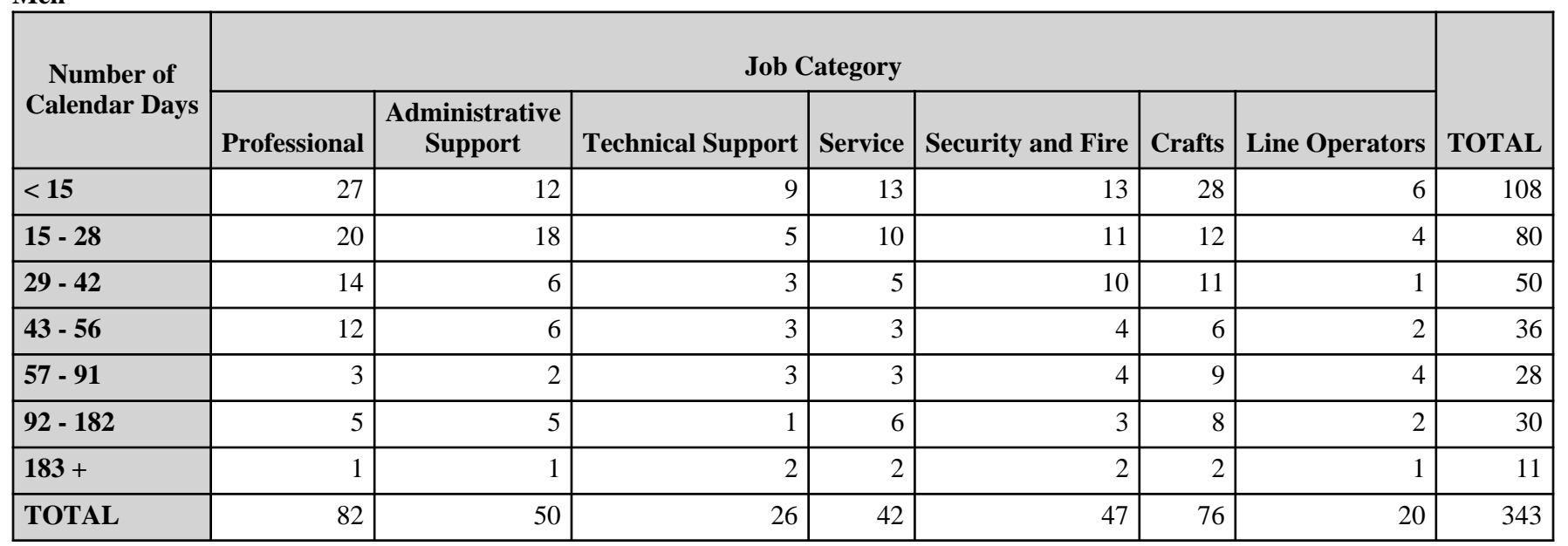

*Only those gender/job category combinations with at least one absence appear in this table. 
Idaho National Laboratory 2010

Absence Data

Appendix G. Number of Diagnoses in Each Diagnostic Category by Gender and Age*

\begin{tabular}{|c|c|c|c|c|c|c|}
\hline & & \multicolumn{5}{|c|}{ Women } \\
\hline & & \multicolumn{4}{|c|}{ Age Group } & \multirow[b]{2}{*}{ TOTAL } \\
\hline & & $16-29$ & $30-39$ & $40-49$ & $50+$ & \\
\hline Diagnostic Category & ICD-9-CM Code & \multirow[b]{2}{*}{0} & \multirow[b]{2}{*}{0} & \multirow[b]{2}{*}{0} & \multirow[b]{2}{*}{4} & \multirow[b]{2}{*}{4} \\
\hline INFECTIOUS \& PARASITIC DISEASES (DIS) & 001-139 & & & & & \\
\hline -Intestinal Infectious Dis & 001-009 & 0 & 0 & 0 & 1 & 1 \\
\hline -Other Bacterial Dis & $030-041$ & 0 & 0 & 0 & 0 & 0 \\
\hline -Syphilis \& Other Venereal Dis & $090-099$ & 0 & 0 & 0 & 2 & 2 \\
\hline -Mycoses & $110-118$ & 0 & 0 & 0 & 1 & 1 \\
\hline MALIGNANT NEOPLASMS & $\begin{array}{l}140-208 \\
209.0-209.3 \\
230-234\end{array}$ & 0 & 0 & 0 & 0 & 0 \\
\hline -Digestive \& Peritoneal & $150-159$ & 0 & 0 & 0 & 0 & 0 \\
\hline -Bone, Connective Tissue, Skin & $170-173,176$ & 0 & 0 & 0 & 0 & 0 \\
\hline -Genitourinary & 179-189 & 0 & 0 & 0 & 0 & 0 \\
\hline -Lymphatic \& Hematopoietic & $200-208$ & 0 & 0 & 0 & 0 & 0 \\
\hline BENIGN \& UNCERTAIN NEOPLASMS & $\begin{array}{l}209.4-209.6 \\
210-229,235-239\end{array}$ & 0 & 0 & 0 & 2 & 2 \\
\hline ENDOCRINE/METABOLIC/IMMUNITY & $240-279$ & 1 & 0 & 0 & 0 & 1 \\
\hline -Other Endocrine Gland Dis & $249-259$ & 0 & 0 & 0 & 0 & 0 \\
\hline -Other Metabolic \& Immunity Disorders & $270-279$ & 1 & 0 & 0 & 0 & 1 \\
\hline MENTAL DISORDERS & 290-319 & 1 & 0 & 2 & 0 & 3 \\
\hline -Non-Psychotic Disorders & $300-302,306-316$ & 1 & 0 & 2 & 0 & 3 \\
\hline NERVOUS SYSTEM (NS) \& SENSE ORGANS & 320-389 & 0 & 1 & 1 & 2 & 4 \\
\hline -Other Disorders of Central NS & $340-349$ & 0 & 1 & 0 & 0 & 1 \\
\hline -Disorders of Peripheral NS & $350-359$ & 0 & 0 & 1 & 2 & 3 \\
\hline -Disorders of Eye & $360-379$ & 0 & 0 & 0 & 0 & 0 \\
\hline -Diseases of Ear \& Mastoid & 380-389 & 0 & 0 & 0 & 0 & 0 \\
\hline CIRCULATORY SYSTEM & $390-459$ & 0 & 0 & 1 & 3 & 4 \\
\hline -Hypertensive Dis & 401-405 & 0 & 0 & 0 & 0 & 0 \\
\hline -Ischemic Heart Dis & $410-414$ & 0 & 0 & 0 & 1 & 1 \\
\hline -Dis of Pulmonary Circulation & $415-417$ & 0 & 0 & 0 & 1 & 1 \\
\hline -Other Heart Dis & $420-429$ & 0 & 0 & 0 & 0 & 0 \\
\hline -Cerebrovascular Dis & $430-438$ & 0 & 0 & 0 & 1 & 1 \\
\hline -Dis of Veins, Lymphatics, Other & 451-459 & 0 & 0 & 1 & 0 & 1 \\
\hline RESPIRATORY SYSTEM & $460-519$ & 1 & 0 & 4 & 8 & 13 \\
\hline -Acute Respiratory Infections & $460-466$ & 0 & 0 & 0 & 0 & 0 \\
\hline -Other Dis Upper Respiratory Tract & $470-478$ & 0 & 0 & 1 & 2 & 3 \\
\hline -Pneumonia \& Influenza & $480-488$ & 1 & 0 & 0 & 3 & 4 \\
\hline -Chronic Obstructive Dis & $490-496$ & 0 & 0 & 1 & 2 & 3 \\
\hline -Lung Dis from External Agents & $500-508$ & 0 & 0 & 0 & 0 & 0 \\
\hline -Other Respiratory Dis & $510-519$ & 0 & 0 & 2 & 1 & 3 \\
\hline
\end{tabular}

(Continued)

*Only those diagnostic categories and gender/age combinations with at least one occurrence appear in this table. 
Idaho National Laboratory 2010

Absence Data

Appendix G. Number of Diagnoses in Each Diagnostic Category by Gender and Age*

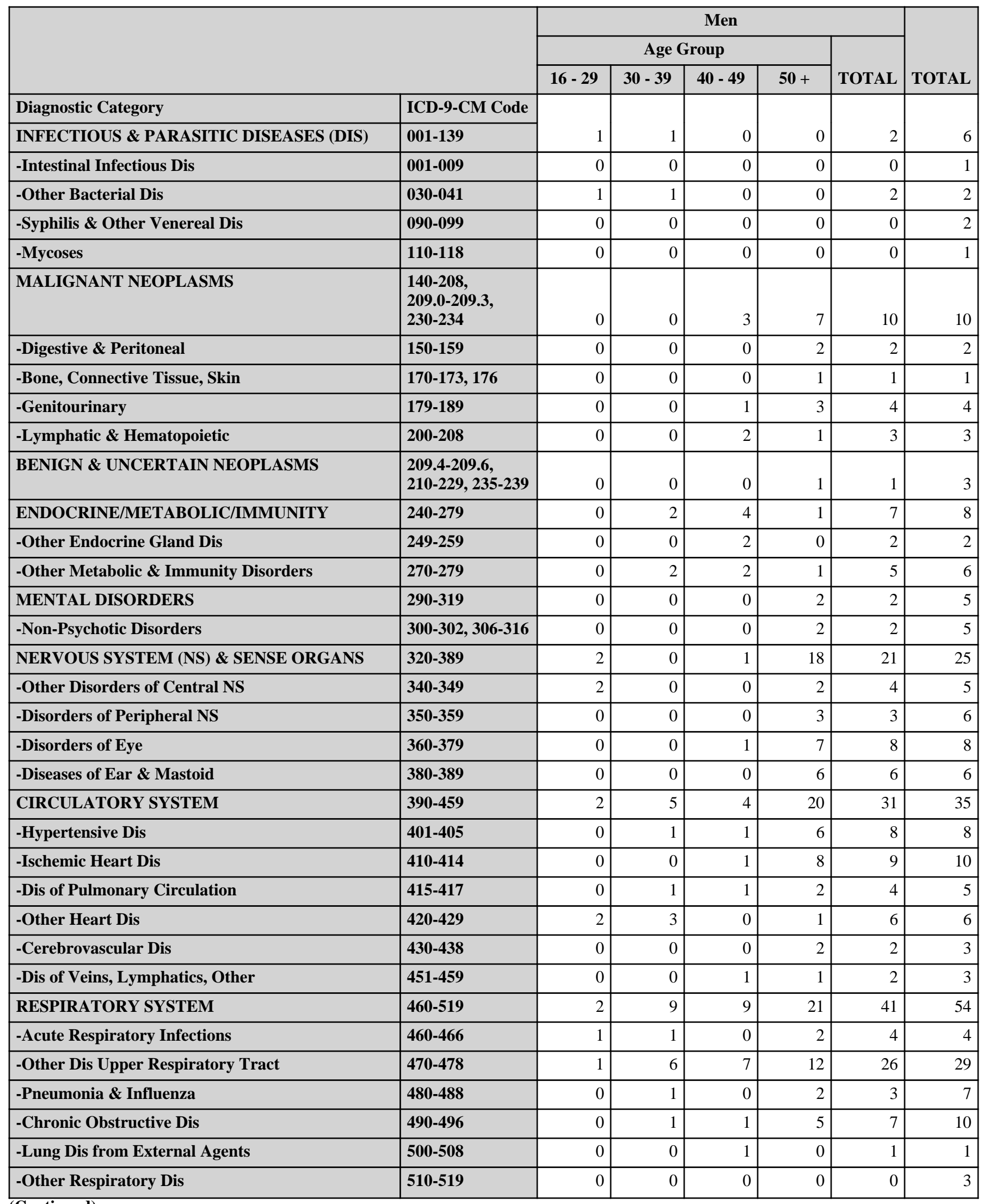

(Continued)

*Only those diagnostic categories and gender/age combinations with at least one occurrence appear in this table. 
Idaho National Laboratory 2010

Absence Data

Appendix G. Number of Diagnoses in Each Diagnostic Category by Gender and Age*

\begin{tabular}{|c|c|c|c|c|c|c|}
\hline & & \multicolumn{5}{|c|}{ Women } \\
\hline & & \multicolumn{4}{|c|}{ Age Group } & \multirow[b]{2}{*}{ TOTAL } \\
\hline & & $16-29$ & $30-39$ & $40-49$ & $50+$ & \\
\hline Diagnostic Category & ICD-9-CM Code & \multirow[b]{2}{*}{2} & \multirow[b]{2}{*}{1} & \multirow[b]{2}{*}{5} & \multirow[b]{2}{*}{8} & \multirow[b]{2}{*}{16} \\
\hline DIGESTIVE SYSTEM & $520-579$ & & & & & \\
\hline -Oral Cavity, Saliva Glands, Jaw & $520-529$ & 0 & 0 & 0 & 1 & 1 \\
\hline -Esophagus, Stomach, Duodenum & $530-538$ & 0 & 0 & 1 & 1 & 2 \\
\hline -Appendicitis & $540-543$ & 0 & 0 & 0 & 0 & 0 \\
\hline -Hernias & $550-553$ & 1 & 0 & 1 & 3 & 5 \\
\hline -Enteritis, Colitis & $555-558$ & 1 & 0 & 0 & 0 & 1 \\
\hline -Other Intestinal Dis & $560-569$ & 0 & 0 & 0 & 1 & 1 \\
\hline -Other Digestive Dis & $570-579$ & 0 & 1 & 3 & 2 & 6 \\
\hline GENITOURINARY SYSTEM & $580-629$ & 0 & 1 & 6 & 5 & 12 \\
\hline -Other Urinary Dis & $590-599$ & 0 & 0 & 1 & 1 & 2 \\
\hline -Male Genital Organ Dis & $600-608$ & 0 & 0 & 0 & 0 & 0 \\
\hline -Breast Disorders & $610-612$ & 0 & 0 & 1 & 1 & 2 \\
\hline -Pelvic Inflammatory Dis & 614-616 & 0 & 0 & 1 & 0 & 1 \\
\hline -Other Female Disorders & $617-629$ & 0 & 1 & 3 & 3 & 7 \\
\hline SKIN AND SUBCUTANEOUS TISSUE & $680-709$ & 0 & 0 & 0 & 0 & 0 \\
\hline -Infections & $680-686$ & 0 & 0 & 0 & 0 & 0 \\
\hline $\begin{array}{l}\text { MUSCULOSKELETAL \& CONNECTIVE } \\
\text { TISSUE }\end{array}$ & 710-739 & 3 & 3 & 8 & 33 & 47 \\
\hline -Arthropathies & $710-719$ & 0 & 1 & 2 & 18 & 21 \\
\hline -Dorsopathies & $720-724$ & 1 & 0 & 4 & 5 & 10 \\
\hline -Rheumatism, Excluding Back & 725-729 & 2 & 2 & 1 & 10 & 15 \\
\hline -Other Dis \& Acquired Deformities & $730-739$ & 0 & 0 & 1 & 0 & 1 \\
\hline $\begin{array}{l}\text { SYMPTOMS, SIGNS, \& ILL-DEFINED } \\
\text { CONDITIONS }\end{array}$ & \begin{tabular}{|l|}
$780-799$ \\
\end{tabular} & 3 & 0 & 1 & 5 & 9 \\
\hline -Symptoms & 780-789 & 2 & 0 & 1 & 4 & 7 \\
\hline -Non-Specific Abnormal Findings & $790-796$ & 1 & 0 & 0 & 1 & 2 \\
\hline INJURY \& POISONING & $800-999$ & 4 & 1 & 30 & 24 & 59 \\
\hline -Fracture - Neck, Trunk & $805-809$ & 3 & 0 & 0 & 0 & 3 \\
\hline -Fracture - Upper Limb & $810-819$ & 0 & 0 & 1 & 2 & 3 \\
\hline -Fracture - Lower Limb & $820-829$ & 0 & 0 & 1 & 1 & 2 \\
\hline -Dislocation & $830-839$ & 0 & 1 & 4 & 3 & 8 \\
\hline -Sprains \& Strains - Back & \begin{tabular}{|l|}
$846-847$ \\
\end{tabular} & 1 & 0 & 6 & 3 & 10 \\
\hline -Sprains \& Strains - Other & $840-845,848$ & 0 & 0 & 9 & 11 & 20 \\
\hline -Intracranial Injury & $850-854$ & 0 & 0 & 0 & 0 & 0 \\
\hline -Open Wound - Head, Neck, Trunk & 870-879 & 0 & 0 & 1 & 0 & 1 \\
\hline -Open Wound - Upper Limb & \begin{tabular}{|l|}
$880-887$ \\
\end{tabular} & 0 & 0 & 0 & 0 & 0 \\
\hline -Open Wound - Lower Limb & $890-897$ & 0 & 0 & 0 & 0 & 0 \\
\hline
\end{tabular}

(Continued)

*Only those diagnostic categories and gender/age combinations with at least one occurrence appear in this table. 
Idaho National Laboratory 2010

Absence Data

Appendix G. Number of Diagnoses in Each Diagnostic Category by Gender and Age*

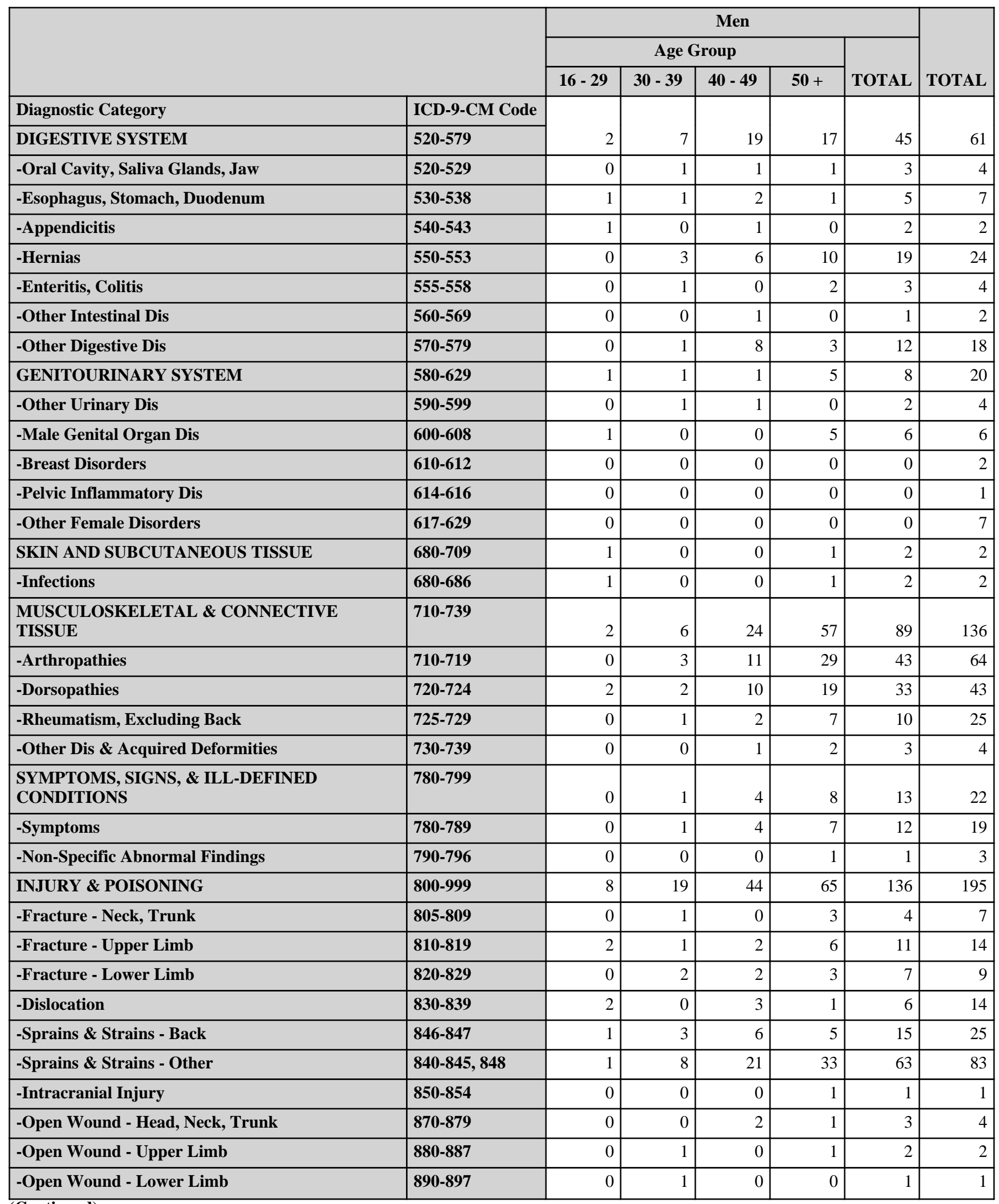

(Continued)

*Only those diagnostic categories and gender/age combinations with at least one occurrence appear in this table. 
Idaho National Laboratory 2010

Absence Data

Appendix G. Number of Diagnoses in Each Diagnostic Category by Gender and Age*

\begin{tabular}{|c|c|c|c|c|c|c|}
\hline & & \multicolumn{5}{|c|}{ Women } \\
\hline & & \multicolumn{4}{|c|}{ Age Group } & \multirow[b]{2}{*}{ TOTAL } \\
\hline & & $16-29$ & $30-39$ & $40-49$ & $50+$ & \\
\hline Diagnostic Category & ICD-9-CM Code & & & & & \\
\hline -Superficial Injury & $910-919$ & 0 & 0 & 0 & 1 & 1 \\
\hline -Contusion & $920-924$ & 0 & 0 & 7 & 1 & 8 \\
\hline -Foreign Body Entering Orifice & 930-939 & 0 & 0 & 0 & 0 & 0 \\
\hline -Complications \& Unspecified Injuries & 958-959 & 0 & 0 & 1 & 1 & 2 \\
\hline -Toxic Effects - Non-medicinal & $980-989$ & 0 & 0 & 0 & 1 & 1 \\
\hline -Unspecified Effects - External Causes & $990-995$ & 0 & 0 & 0 & 0 & 0 \\
\hline -Complications of Surgical/Medical Care & 996-999 & 0 & 0 & 0 & 0 & 0 \\
\hline $\begin{array}{l}\text { HEALTH STATUS/HEALTH SERVICE } \\
\text { CONTACT }\end{array}$ & V01-V89 & 2 & 1 & 1 & 1 & 5 \\
\hline -Health Services Reproduction/Development & V20-V29 & 0 & 1 & 0 & 0 & 1 \\
\hline -Specific Procedures/Aftercare & V50-V59 & 1 & 0 & 0 & 1 & 2 \\
\hline -Examination \& Investigation & V70-V82 & 1 & 0 & 1 & 0 & 2 \\
\hline
\end{tabular}

*Only those diagnostic categories and gender/age combinations with at least one occurrence appear in this table. 
Idaho National Laboratory 2010

Absence Data

Appendix G. Number of Diagnoses in Each Diagnostic Category by Gender and Age*

\begin{tabular}{|c|c|c|c|c|c|c|c|}
\hline & & \multicolumn{5}{|c|}{ Men } & \multirow[b]{3}{*}{ TOTAL } \\
\hline & & \multicolumn{4}{|c|}{ Age Group } & \multirow[b]{2}{*}{ TOTAL } & \\
\hline & & $16-29$ & $30-39$ & $40-49$ & $50+$ & & \\
\hline -Superficial Injury & 910-919 & 1 & 0 & 0 & 1 & 2 & 3 \\
\hline -Contusion & $920-924$ & 0 & 0 & 2 & 6 & 8 & 16 \\
\hline -Complications \& Unspecified Injuries & 958-959 & 0 & 1 & 1 & 2 & 4 & 6 \\
\hline -Toxic Effects - Non-medicinal & 980-989 & 0 & 0 & 0 & 0 & 0 & 1 \\
\hline -Unspecified Effects - External Causes & $990-995$ & 1 & 0 & 1 & 0 & 2 & 2 \\
\hline -Complications of Surgical/Medical Care & 996-999 & 0 & 1 & 4 & 1 & 6 & 6 \\
\hline -Specific Procedures/Aftercare & V50-V59 & 0 & 0 & 0 & 0 & 0 & 2 \\
\hline -Examination \& Investigation & V70-V82 & 0 & 0 & 1 & 3 & 4 & 6 \\
\hline
\end{tabular}

\begin{tabular}{|c|c|c|c|c|c|c|c|c|c|c|c|}
\hline & \multicolumn{5}{|c|}{ Women } & \multicolumn{5}{|c|}{ Men } & \multirow[b]{3}{*}{ TOTAL } \\
\hline & \multicolumn{4}{|c|}{ Age Group } & \multirow[b]{2}{*}{ TOTAL } & \multicolumn{4}{|c|}{ Age Group } & \multirow[b]{2}{*}{ TOTAL } & \\
\hline & $16-29$ & $30-39$ & $40-49$ & $50+$ & & $16-29$ & $30-39$ & $40-49$ & $50+$ & & \\
\hline Diagnostic Category & \multirow[b]{2}{*}{17} & \multirow[b]{2}{*}{8} & \multirow[b]{2}{*}{59} & \multirow[b]{2}{*}{95} & \multirow[b]{2}{*}{179} & \multirow[b]{2}{*}{22} & \multirow[b]{2}{*}{51} & \multirow[b]{2}{*}{114} & \multirow[b]{2}{*}{226} & \multirow[b]{2}{*}{413} & \multirow[b]{2}{*}{592} \\
\hline Total & & & & & & & & & & & \\
\hline
\end{tabular}

*Only those diagnostic categories and gender/age combinations with at least one occurrence appear in this table. 
Idaho National Laboratory 2010

Absence Data

Appendix H. Total Number of Calendar Days Absent in Each Diagnostic Category by Gender and Age*

\begin{tabular}{|c|c|c|c|c|c|c|}
\hline & & \multicolumn{5}{|c|}{ Women } \\
\hline & & \multicolumn{4}{|c|}{ Age Group } & \multirow[b]{2}{*}{ TOTAL } \\
\hline & & $16-29$ & $30-39$ & $40-49$ & $50+$ & \\
\hline Diagnostic Category & ICD-9-CM Code & \multirow[b]{2}{*}{0} & \multirow[b]{2}{*}{0} & \multirow[b]{2}{*}{0} & \multirow[b]{2}{*}{139} & \multirow[b]{2}{*}{139} \\
\hline INFECTIOUS \& PARASITIC DISEASES (DIS) & 001-139 & & & & & \\
\hline MALIGNANT NEOPLASMS & $\begin{array}{l}140-208 \\
209.0-209.3 \\
230-234\end{array}$ & 0 & 0 & 0 & 0 & 0 \\
\hline BENIGN \& UNCERTAIN NEOPLASMS & \begin{tabular}{|l|}
$209.4-209.6$ \\
$210-229,235-239$
\end{tabular} & 0 & 0 & 0 & 239 & 239 \\
\hline ENDOCRINE/METABOLIC/IMMUNITY & $240-279$ & 32 & 0 & 0 & 0 & 32 \\
\hline MENTAL DISORDERS & $290-319$ & 58 & 0 & 27 & 0 & 85 \\
\hline NERVOUS SYSTEM (NS) \& SENSE ORGANS & $320-389$ & 0 & 1 & 14 & 85 & 100 \\
\hline CIRCULATORY SYSTEM & $390-459$ & 0 & 0 & 13 & 49 & 62 \\
\hline RESPIRATORY SYSTEM & $460-519$ & 12 & 0 & 99 & 107 & 218 \\
\hline DIGESTIVE SYSTEM & $520-579$ & 9 & 21 & 92 & 131 & 253 \\
\hline GENITOURINARY SYSTEM & $580-629$ & 0 & 16 & 167 & 74 & 257 \\
\hline SKIN AND SUBCUTANEOUS TISSUE & $680-709$ & 0 & 0 & 0 & 0 & 0 \\
\hline $\begin{array}{l}\text { MUSCULOSKELETAL \& CONNECTIVE } \\
\text { TISSUE }\end{array}$ & $710-739$ & 36 & 143 & 327 & 1,787 & 2,293 \\
\hline $\begin{array}{l}\text { SYMPTOMS, SIGNS, \& ILL-DEFINED } \\
\text { CONDITIONS }\end{array}$ & $780-799$ & 13 & 0 & 2 & 32 & 47 \\
\hline INJURY \& POISONING & $800-999$ & 82 & 126 & 1,154 & 745 & 2,107 \\
\hline
\end{tabular}

\footnotetext{
*Absences with >1 ICD-9-CM code in the same diagnostic category were counted only once. Only those diagnostic categories and gender/age combinations with at least one occurrence appear in this table.
} 
Idaho National Laboratory 2010

Absence Data

Appendix H. Total Number of Calendar Days Absent in Each Diagnostic Category by Gender and Age*

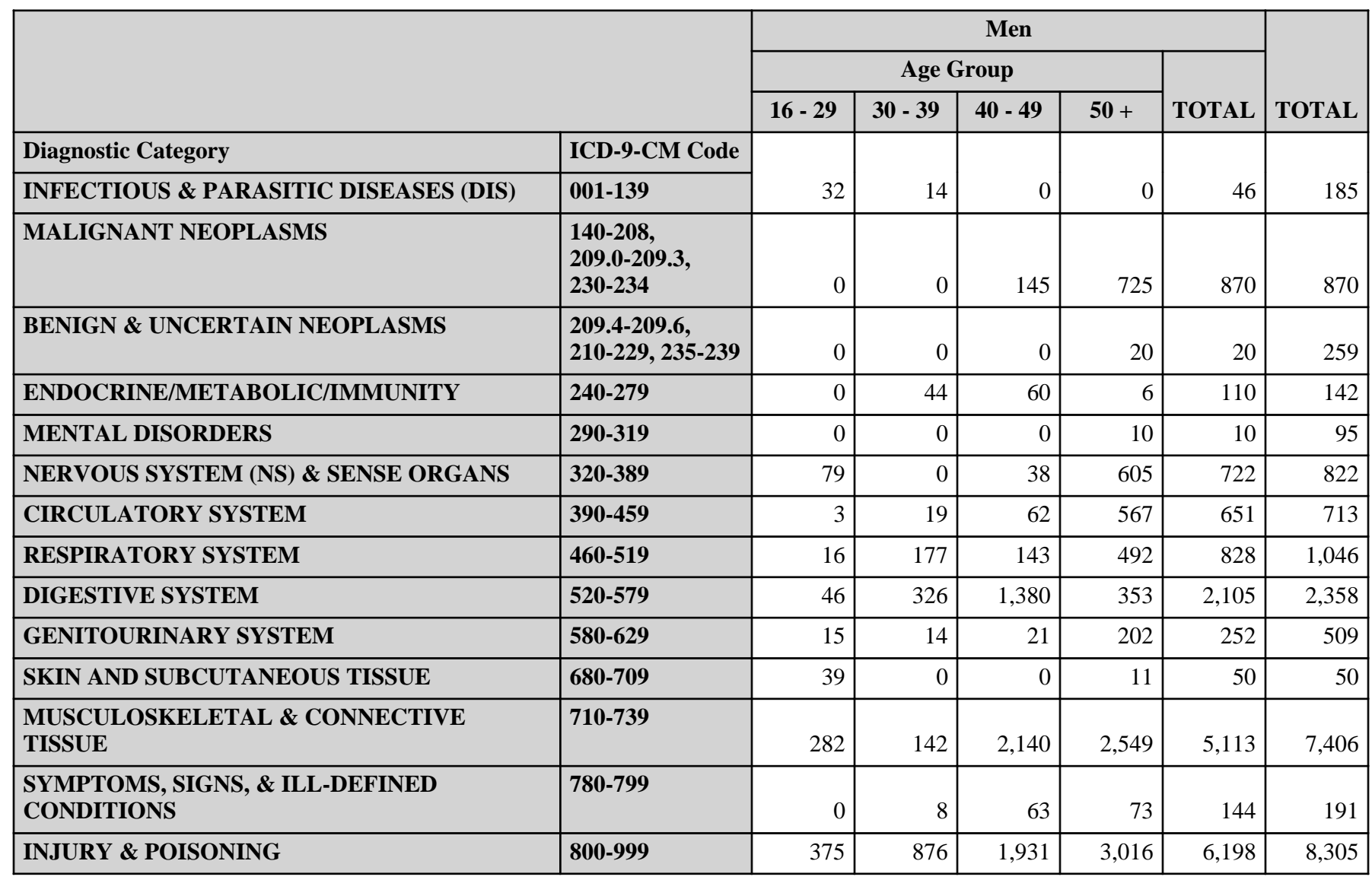

\footnotetext{
*Absences with >1 ICD-9-CM code in the same diagnostic category were counted only once. Only those diagnostic categories and gender/age combinations with at least one occurrence appear in this table.
} 
Idaho National Laboratory 2010

Absence Data

Appendix I. Number of Diagnoses in Each Diagnostic Category by Gender and Job Category*

\begin{tabular}{|c|c|c|c|c|c|}
\hline & & \multicolumn{4}{|c|}{ Women } \\
\hline & & \multicolumn{4}{|c|}{ Job Category } \\
\hline & & Professional & $\begin{array}{c}\text { Administrative } \\
\text { Support }\end{array}$ & Technical Support & Service \\
\hline Diagnostic Category & ICD-9-CM Code & \multirow[b]{2}{*}{0} & \multirow[b]{2}{*}{4} & \multirow[b]{2}{*}{0} & \multirow[b]{2}{*}{0} \\
\hline INFECTIOUS \& PARASITIC DISEASES (DIS) & 001-139 & & & & \\
\hline -Syphilis \& Other Venereal Dis & 090-099 & 0 & 2 & 0 & 0 \\
\hline -Mycoses & $110-118$ & 0 & 1 & 0 & 0 \\
\hline BENIGN \& UNCERTAIN NEOPLASMS & \begin{tabular}{|l|}
$209.4-209.6$ \\
$210-229$, \\
$235-239$
\end{tabular} & 1 & 0 & 0 & 0 \\
\hline ENDOCRINE/METABOLIC/IMMUNITY & 240-279 & 0 & 0 & 1 & 0 \\
\hline -Non-Psychotic Disorders & $300-302,306-316$ & 0 & 2 & 1 & 0 \\
\hline NERVOUS SYSTEM (NS) \& SENSE ORGANS & $320-389$ & 0 & 3 & 0 & 1 \\
\hline -Other Disorders of Central NS & $340-349$ & 0 & 1 & 0 & 0 \\
\hline -Disorders of Peripheral NS & $350-359$ & 0 & 2 & 0 & 1 \\
\hline CIRCULATORY SYSTEM & $390-459$ & 1 & 2 & 0 & 0 \\
\hline -Ischemic Heart Dis & $410-414$ & 0 & 1 & 0 & 0 \\
\hline -Dis of Pulmonary Circulation & $415-417$ & 0 & 1 & 0 & 0 \\
\hline -Cerebrovascular Dis & $430-438$ & 1 & 0 & 0 & 0 \\
\hline DIGESTIVE SYSTEM & $520-579$ & 1 & 9 & 2 & 2 \\
\hline -Oral Cavity, Saliva Glands, Jaw & $520-529$ & 0 & 0 & 0 & 1 \\
\hline -Esophagus, Stomach, Duodenum & $530-538$ & 1 & 1 & 0 & 0 \\
\hline -Hernias & $550-553$ & 0 & 2 & 2 & 0 \\
\hline -Enteritis, Colitis & $555-558$ & 0 & 1 & 0 & 0 \\
\hline -Other Intestinal Dis & $560-569$ & 0 & 1 & 0 & 0 \\
\hline -Other Digestive Dis & $570-579$ & 0 & 4 & 0 & 1 \\
\hline GENITOURINARY SYSTEM & $580-629$ & 0 & 8 & 2 & 0 \\
\hline -Other Urinary Dis & $590-599$ & 0 & 2 & 0 & 0 \\
\hline -Breast Disorders & $610-612$ & 0 & 2 & 0 & 0 \\
\hline -Pelvic Inflammatory Dis & 614-616 & 0 & 0 & 0 & 0 \\
\hline -Other Female Disorders & $617-629$ & 0 & 4 & 2 & 0 \\
\hline $\begin{array}{l}\text { MUSCULOSKELETAL \& CONNECTIVE } \\
\text { TISSUE }\end{array}$ & $710-739$ & 6 & 27 & 0 & 7 \\
\hline
\end{tabular}

(Continued)

*Only those diagnostic categories and gender/job category combinations with at least one occurrence appear in this table. 
Idaho National Laboratory 2010

Absence Data

Appendix I. Number of Diagnoses in Each Diagnostic Category by Gender and Job Category*

\begin{tabular}{|c|c|c|c|c|c|}
\hline & & \multicolumn{4}{|c|}{ Women } \\
\hline & & \multicolumn{3}{|c|}{ Job Category } & \multirow[b]{2}{*}{ TOTAL } \\
\hline & & Security and Fire & Crafts & Line Operators & \\
\hline Diagnostic Category & ICD-9-CM Code & \multirow[b]{2}{*}{0} & \multirow[b]{2}{*}{0} & \multirow[b]{2}{*}{0} & \multirow[b]{2}{*}{4} \\
\hline INFECTIOUS \& PARASITIC DISEASES (DIS) & 001-139 & & & & \\
\hline -Intestinal Infectious Dis & 001-009 & 0 & 0 & 0 & 1 \\
\hline -Syphilis \& Other Venereal Dis & 090-099 & 0 & 0 & 0 & 2 \\
\hline -Mycoses & 110-118 & 0 & 0 & 0 & 1 \\
\hline BENIGN \& UNCERTAIN NEOPLASMS & $\begin{array}{l}209.4-209.6 \\
210-229,235-239\end{array}$ & 0 & 1 & 0 & 2 \\
\hline ENDOCRINE/METABOLIC/IMMUNITY & $240-279$ & 0 & 0 & 0 & 1 \\
\hline -Other Metabolic \& Immunity Disorders & $270-279$ & 0 & 0 & 0 & 1 \\
\hline MENTAL DISORDERS & $290-319$ & 0 & 0 & 0 & 3 \\
\hline -Non-Psychotic Disorders & $300-302,306-316$ & 0 & 0 & 0 & 3 \\
\hline NERVOUS SYSTEM (NS) \& SENSE ORGANS & $320-389$ & 0 & 0 & 0 & 4 \\
\hline -Other Disorders of Central NS & $340-349$ & 0 & 0 & 0 & 1 \\
\hline -Disorders of Peripheral NS & $350-359$ & 0 & 0 & 0 & 3 \\
\hline CIRCULATORY SYSTEM & $390-459$ & 1 & 0 & 0 & 4 \\
\hline -Ischemic Heart Dis & $410-414$ & 0 & 0 & 0 & 1 \\
\hline -Dis of Pulmonary Circulation & $415-417$ & 0 & 0 & 0 & 1 \\
\hline -Cerebrovascular Dis & $430-438$ & 0 & 0 & 0 & 1 \\
\hline -Dis of Veins, Lymphatics, Other & 451-459 & 1 & 0 & 0 & 1 \\
\hline RESPIRATORY SYSTEM & $460-519$ & 1 & 2 & 0 & 13 \\
\hline -Other Dis Upper Respiratory Tract & $470-478$ & 0 & 1 & 0 & 3 \\
\hline -Pneumonia \& Influenza & $480-488$ & 0 & 1 & 0 & 4 \\
\hline -Chronic Obstructive Dis & $490-496$ & 0 & 0 & 0 & 3 \\
\hline -Other Respiratory Dis & $510-519$ & 1 & 0 & 0 & 3 \\
\hline DIGESTIVE SYSTEM & $520-579$ & 0 & 2 & 0 & 16 \\
\hline -Oral Cavity, Saliva Glands, Jaw & $520-529$ & 0 & 0 & 0 & 1 \\
\hline -Esophagus, Stomach, Duodenum & $530-538$ & 0 & 0 & 0 & 2 \\
\hline -Hernias & $550-553$ & 0 & 1 & 0 & 5 \\
\hline -Enteritis, Colitis & $555-558$ & 0 & 0 & 0 & 1 \\
\hline -Other Intestinal Dis & $560-569$ & 0 & 0 & 0 & 1 \\
\hline -Other Digestive Dis & $570-579$ & 0 & 1 & 0 & $\overline{6}$ \\
\hline GENITOURINARY SYSTEM & $580-629$ & 1 & 1 & 0 & 12 \\
\hline -Other Urinary Dis & $590-599$ & 0 & 0 & 0 & 2 \\
\hline -Breast Disorders & $610-612$ & 0 & 0 & 0 & 2 \\
\hline -Pelvic Inflammatory Dis & 614-616 & 0 & 1 & 0 & 1 \\
\hline -Other Female Disorders & 617-629 & 1 & 0 & 0 & 7 \\
\hline $\begin{array}{l}\text { MUSCULOSKELETAL \& CONNECTIVE } \\
\text { TISSUE }\end{array}$ & $710-739$ & 4 & 2 & 1 & 47 \\
\hline
\end{tabular}

(Continued)

*Only those diagnostic categories and gender/job category combinations with at least one occurrence appear in this table. 
Idaho National Laboratory 2010

Absence Data

Appendix I. Number of Diagnoses in Each Diagnostic Category by Gender and Job Category*

\begin{tabular}{|c|c|c|c|c|c|}
\hline & & \multicolumn{4}{|c|}{ Women } \\
\hline & & \multicolumn{4}{|c|}{ Job Category } \\
\hline & & Professional & $\begin{array}{c}\text { Administrative } \\
\text { Support }\end{array}$ & Technical Support & Service \\
\hline Diagnostic Category & ICD-9-CM Code & \multirow[b]{2}{*}{1} & \multirow[b]{2}{*}{13} & \multirow[b]{2}{*}{0} & \multirow[b]{2}{*}{6} \\
\hline -Arthropathies & $710-719$ & & & & \\
\hline -Rheumatism, Excluding Back & $725-729$ & 3 & 8 & 0 & 0 \\
\hline -Other Dis \& Acquired Deformities & $730-739$ & 0 & 0 & 0 & 0 \\
\hline $\begin{array}{l}\text { SYMPTOMS, SIGNS, \& ILL-DEFINED } \\
\text { CONDITIONS }\end{array}$ & $780-799$ & 2 & 3 & 3 & 1 \\
\hline -Symptoms & $780-789$ & 1 & 3 & 2 & 1 \\
\hline -Fracture - Neck, Trunk & 805-809 & 0 & 3 & 0 & 0 \\
\hline -Fracture - Upper Limb & $810-819$ & 2 & 1 & 0 & 0 \\
\hline -Fracture - Lower Limb & $820-829$ & 0 & 2 & 0 & 0 \\
\hline -Dislocation & 830-839 & 0 & 5 & 0 & 3 \\
\hline -Sprains \& Strains - Back & 846-847 & 0 & 4 & 1 & 2 \\
\hline -Sprains \& Strains - Other & $840-845,848$ & 2 & 14 & 2 & 2 \\
\hline -Open Wound - Head, Neck, Trunk & $870-879$ & 0 & 0 & 0 & 0 \\
\hline -Superficial Injury & $910-919$ & 0 & 0 & 0 & 1 \\
\hline -Specific Procedures/Aftercare & V50-V59 & 0 & 1 & 0 & 0 \\
\hline -Examination \& Investigation & V70-V82 & 0 & 0 & 1 & 0 \\
\hline
\end{tabular}

*Only those diagnostic categories and gender/job category combinations with at least one occurrence appear in this table. 
Idaho National Laboratory 2010

Absence Data

Appendix I. Number of Diagnoses in Each Diagnostic Category by Gender and Job Category*

\begin{tabular}{|c|c|c|c|c|c|}
\hline & & \multicolumn{4}{|c|}{ Women } \\
\hline & & \multicolumn{3}{|c|}{ Job Category } & \multirow[b]{2}{*}{ TOTAL } \\
\hline & & Security and Fire & Crafts & Line Operators & \\
\hline Diagnostic Category & ICD-9-CM Code & \multirow[b]{2}{*}{0} & \multirow[b]{2}{*}{1} & \multirow[b]{2}{*}{0} & \multirow[b]{2}{*}{21} \\
\hline -Arthropathies & $710-719$ & & & & \\
\hline -Dorsopathies & $720-724$ & 1 & 0 & 0 & 10 \\
\hline -Rheumatism, Excluding Back & 725-729 & 2 & 1 & 1 & 15 \\
\hline -Other Dis \& Acquired Deformities & $730-739$ & 1 & 0 & 0 & 1 \\
\hline $\begin{array}{l}\text { SYMPTOMS, SIGNS, \& ILL-DEFINED } \\
\text { CONDITIONS }\end{array}$ & 780-799 & 0 & 0 & 0 & 9 \\
\hline -Symptoms & 780-789 & 0 & 0 & 0 & 7 \\
\hline -Non-Specific Abnormal Findings & $790-796$ & 0 & 0 & 0 & 2 \\
\hline INJURY \& POISONING & 800-999 & 0 & 6 & 0 & 59 \\
\hline -Fracture - Neck, Trunk & 805-809 & 0 & 0 & 0 & 3 \\
\hline -Fracture - Upper Limb & 810-819 & 0 & 0 & 0 & 3 \\
\hline -Fracture - Lower Limb & $820-829$ & 0 & 0 & 0 & 2 \\
\hline -Dislocation & 830-839 & 0 & 0 & 0 & 8 \\
\hline -Sprains \& Strains - Back & 846-847 & 0 & 3 & 0 & 10 \\
\hline -Sprains \& Strains - Other & $840-845,848$ & 0 & 0 & 0 & 20 \\
\hline -Open Wound - Head, Neck, Trunk & 870-879 & 0 & 1 & 0 & 1 \\
\hline -Superficial Injury & 910-919 & 0 & 0 & 0 & 1 \\
\hline -Contusion & 920-924 & 0 & 1 & 0 & 8 \\
\hline -Complications \& Unspecified Injuries & 958-959 & 0 & 1 & 0 & 2 \\
\hline -Toxic Effects - Non-medicinal & 980-989 & 0 & 0 & 0 & 1 \\
\hline $\begin{array}{l}\text { HEALTH STATUS/HEALTH SERVICE } \\
\text { CONTACT }\end{array}$ & V01-V89 & 1 & 1 & 0 & 5 \\
\hline -Health Services Reproduction/Development & V20-V29 & 0 & 0 & 0 & 1 \\
\hline -Specific Procedures/Aftercare & V50-V59 & 0 & 1 & 0 & 2 \\
\hline -Examination \& Investigation & V70-V82 & 1 & 0 & 0 & 2 \\
\hline
\end{tabular}

\begin{tabular}{|c|c|c|c|c|c|c|c|c|}
\hline & \multicolumn{8}{|c|}{ Women } \\
\hline & \multicolumn{7}{|c|}{ Job Category } & \multirow[b]{2}{*}{ TOTAI } \\
\hline & Professional & $\begin{array}{c}\text { Administrative } \\
\text { Support }\end{array}$ & $\begin{array}{c}\text { Technical } \\
\text { Support }\end{array}$ & Service & $\begin{array}{c}\text { Security and } \\
\text { Fire }\end{array}$ & Crafts & $\begin{array}{c}\text { Line } \\
\text { Operators }\end{array}$ & \\
\hline Diagnostic Category & \multirow[b]{2}{*}{19} & \multirow[b]{2}{*}{97} & \multirow[b]{2}{*}{18} & \multirow[b]{2}{*}{21} & \multirow[b]{2}{*}{8} & \multirow[b]{2}{*}{15} & \multirow[b]{2}{*}{1} & \multirow[b]{2}{*}{179} \\
\hline Total & & & & & & & & \\
\hline
\end{tabular}

*Only those diagnostic categories and gender/job category combinations with at least one occurrence appear in this table. 
Idaho National Laboratory 2010

Absence Data

Appendix I. Number of Diagnoses in Each Diagnostic Category by Gender and Job Category*

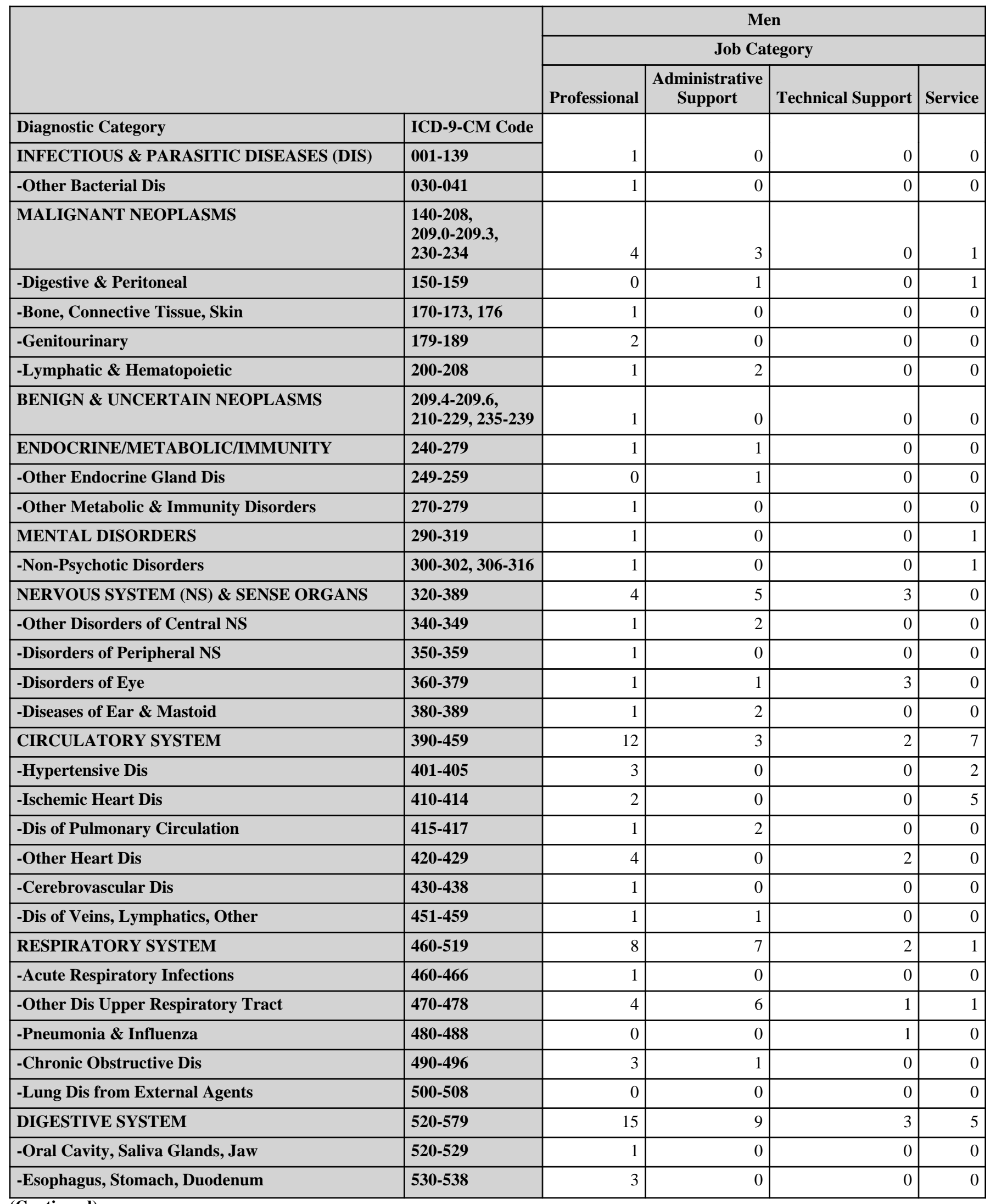

(Continued)

*Only those diagnostic categories and gender/job category combinations with at least one occurrence appear in this table. 
Idaho National Laboratory 2010

Absence Data

Appendix I. Number of Diagnoses in Each Diagnostic Category by Gender and Job Category*

\begin{tabular}{|c|c|c|c|c|c|}
\hline & & \multicolumn{4}{|c|}{ Men } \\
\hline & & \multicolumn{3}{|c|}{ Job Category } & \multirow[b]{2}{*}{ TOTAL } \\
\hline & & Security and Fire & Crafts & Line Operators & \\
\hline Diagnostic Category & ICD-9-CM Code & \multirow[b]{2}{*}{0} & \multirow[b]{2}{*}{0} & \multirow[b]{2}{*}{1} & \multirow[b]{2}{*}{2} \\
\hline INFECTIOUS \& PARASITIC DISEASES (DIS) & 001-139 & & & & \\
\hline -Other Bacterial Dis & $030-041$ & 0 & 0 & 1 & 2 \\
\hline MALIGNANT NEOPLASMS & $\begin{array}{l}140-208 \\
209.0-209.3 \\
230-234\end{array}$ & 0 & 2 & 0 & 10 \\
\hline -Digestive \& Peritoneal & $150-159$ & 0 & 0 & 0 & 2 \\
\hline -Bone, Connective Tissue, Skin & $170-173,176$ & 0 & 0 & 0 & 1 \\
\hline -Genitourinary & 179-189 & 0 & 2 & 0 & 4 \\
\hline -Lymphatic \& Hematopoietic & $200-208$ & 0 & 0 & 0 & 3 \\
\hline BENIGN \& UNCERTAIN NEOPLASMS & $\begin{array}{l}209.4-209.6 \\
210-229,235-239\end{array}$ & 0 & 0 & 0 & 1 \\
\hline ENDOCRINE/METABOLIC/IMMUNITY & $240-279$ & 1 & 3 & 1 & 7 \\
\hline -Other Endocrine Gland Dis & $249-259$ & 0 & 1 & 0 & 2 \\
\hline -Other Metabolic \& Immunity Disorders & $270-279$ & 1 & 2 & 1 & 5 \\
\hline MENTAL DISORDERS & 290-319 & 0 & 0 & 0 & 2 \\
\hline -Non-Psychotic Disorders & $300-302,306-316$ & 0 & 0 & 0 & 2 \\
\hline NERVOUS SYSTEM (NS) \& SENSE ORGANS & $320-389$ & 1 & 6 & 2 & 21 \\
\hline -Other Disorders of Central NS & $340-349$ & 0 & 1 & 0 & 4 \\
\hline -Disorders of Peripheral NS & $350-359$ & 0 & 2 & 0 & 3 \\
\hline -Disorders of Eye & $360-379$ & 1 & 1 & 1 & 8 \\
\hline -Diseases of Ear \& Mastoid & 380-389 & 0 & 2 & 1 & 6 \\
\hline CIRCULATORY SYSTEM & $390-459$ & 2 & 4 & 1 & 31 \\
\hline -Hypertensive Dis & $401-405$ & 1 & 2 & 0 & 8 \\
\hline -Ischemic Heart Dis & $410-414$ & 0 & 1 & 1 & 9 \\
\hline -Dis of Pulmonary Circulation & $415-417$ & 1 & 0 & 0 & 4 \\
\hline -Other Heart Dis & $420-429$ & 0 & 0 & 0 & 6 \\
\hline -Cerebrovascular Dis & $430-438$ & 0 & 1 & 0 & 2 \\
\hline -Dis of Veins, Lymphatics, Other & 451-459 & 0 & 0 & 0 & 2 \\
\hline RESPIRATORY SYSTEM & $460-519$ & 8 & 14 & 1 & 41 \\
\hline -Acute Respiratory Infections & $460-466$ & 1 & 2 & 0 & 4 \\
\hline -Other Dis Upper Respiratory Tract & $470-478$ & 6 & 8 & 0 & 26 \\
\hline -Pneumonia \& Influenza & $480-488$ & 0 & 1 & 1 & 3 \\
\hline -Chronic Obstructive Dis & $490-496$ & 0 & 3 & 0 & 7 \\
\hline -Lung Dis from External Agents & $500-508$ & 1 & 0 & 0 & 1 \\
\hline DIGESTIVE SYSTEM & $520-579$ & 4 & 8 & 1 & 45 \\
\hline -Oral Cavity, Saliva Glands, Jaw & $520-529$ & 1 & 1 & 0 & 3 \\
\hline -Esophagus, Stomach, Duodenum & $530-538$ & 0 & 2 & 0 & 5 \\
\hline
\end{tabular}

(Continued)

*Only those diagnostic categories and gender/job category combinations with at least one occurrence appear in this table. 
Idaho National Laboratory 2010

Absence Data

Appendix I. Number of Diagnoses in Each Diagnostic Category by Gender and Job Category*

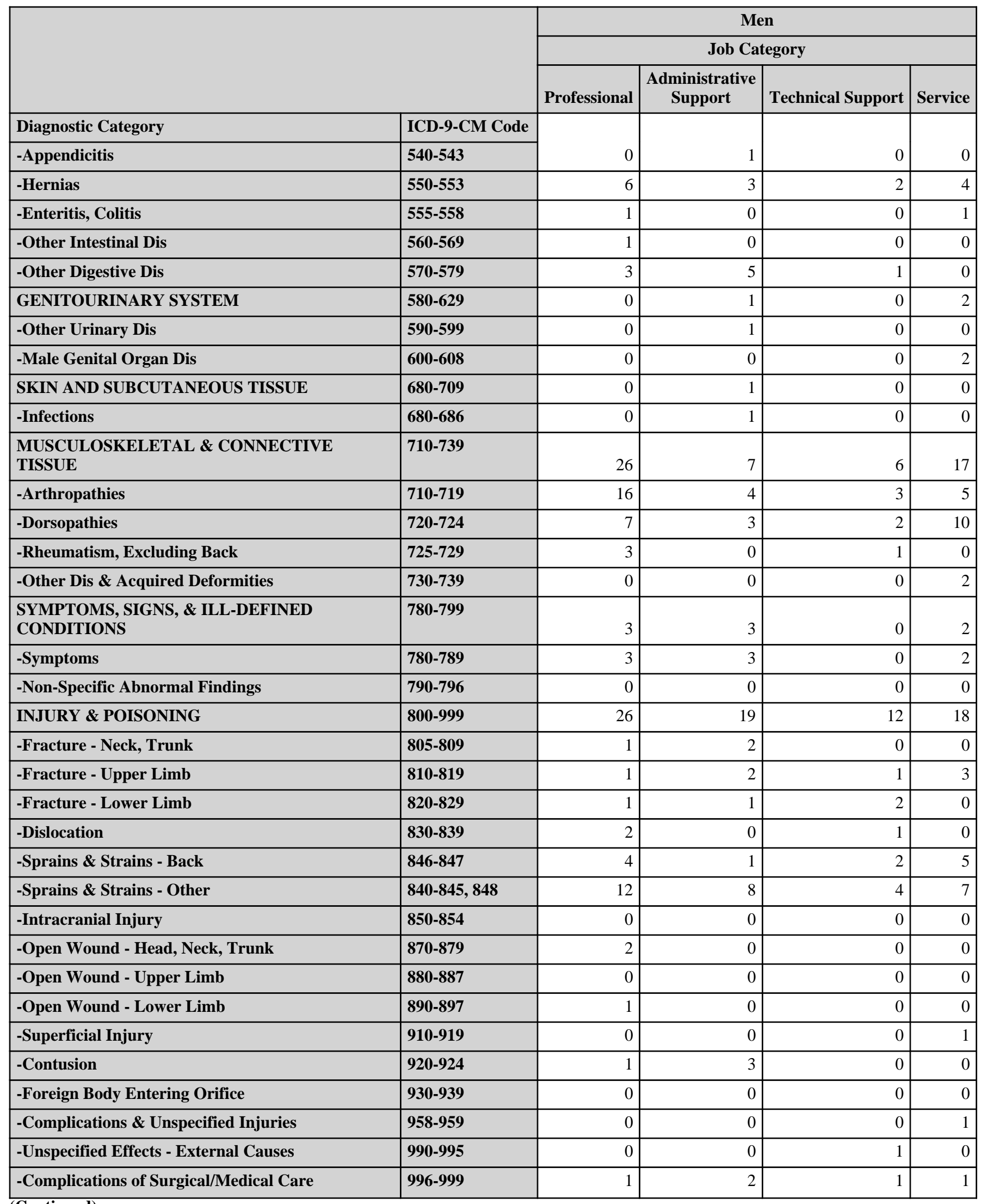

(Continued)

*Only those diagnostic categories and gender/job category combinations with at least one occurrence appear in this table. 
Idaho National Laboratory 2010

Absence Data

Appendix I. Number of Diagnoses in Each Diagnostic Category by Gender and Job Category*

\begin{tabular}{|c|c|c|c|c|c|}
\hline & & \multicolumn{4}{|c|}{ Men } \\
\hline & & \multicolumn{3}{|c|}{ Job Category } & \multirow[b]{2}{*}{ TOTAL } \\
\hline & & Security and Fire & Crafts & Line Operators & \\
\hline Diagnostic Category & ICD-9-CM Code & \multirow[b]{2}{*}{0} & \multirow[b]{2}{*}{1} & \multirow[b]{2}{*}{0} & \multirow[b]{2}{*}{2} \\
\hline -Appendicitis & $540-543$ & & & & \\
\hline -Hernias & $550-553$ & 2 & 2 & 0 & 19 \\
\hline -Enteritis, Colitis & $555-558$ & 0 & 1 & 0 & 3 \\
\hline -Other Intestinal Dis & $560-569$ & 0 & 0 & 0 & 1 \\
\hline -Other Digestive Dis & $570-579$ & 1 & 1 & 1 & 12 \\
\hline GENITOURINARY SYSTEM & $580-629$ & 1 & 4 & 0 & 8 \\
\hline -Other Urinary Dis & $590-599$ & 0 & 1 & 0 & 2 \\
\hline -Male Genital Organ Dis & $600-608$ & 1 & 3 & 0 & $\overline{6}$ \\
\hline SKIN AND SUBCUTANEOUS TISSUE & 680-709 & 0 & 1 & 0 & 2 \\
\hline -Infections & $680-686$ & 0 & 1 & 0 & 2 \\
\hline $\begin{array}{l}\text { MUSCULOSKELETAL \& CONNECTIVE } \\
\text { TISSUE }\end{array}$ & $710-739$ & 11 & 14 & 8 & 89 \\
\hline -Arthropathies & $710-719$ & 8 & 4 & 3 & 43 \\
\hline -Dorsopathies & $720-724$ & 2 & 5 & 4 & 33 \\
\hline -Rheumatism, Excluding Back & 725-729 & 1 & 4 & 1 & 10 \\
\hline -Other Dis \& Acquired Deformities & 730-739 & 0 & 1 & 0 & 3 \\
\hline $\begin{array}{l}\text { SYMPTOMS, SIGNS, \& ILL-DEFINED } \\
\text { CONDITIONS }\end{array}$ & $780-799$ & 2 & 3 & 0 & 13 \\
\hline -Symptoms & 780-789 & 2 & 2 & 0 & 12 \\
\hline -Non-Specific Abnormal Findings & $790-796$ & 0 & 1 & 0 & 1 \\
\hline INJURY \& POISONING & $800-999$ & 23 & 30 & 8 & 136 \\
\hline -Fracture - Neck, Trunk & $805-809$ & 1 & 0 & 0 & 4 \\
\hline -Fracture - Upper Limb & 810-819 & 1 & 3 & 0 & 11 \\
\hline -Fracture - Lower Limb & $820-829$ & 2 & 1 & 0 & 7 \\
\hline -Dislocation & $830-839$ & 0 & 2 & 1 & 6 \\
\hline -Sprains \& Strains - Back & 846-847 & 1 & 2 & 0 & 15 \\
\hline -Sprains \& Strains - Other & $840-845,848$ & 11 & 17 & 4 & 63 \\
\hline -Intracranial Injury & $850-854$ & 1 & 0 & 0 & 1 \\
\hline -Open Wound - Head, Neck, Trunk & 870-879 & 1 & 0 & 0 & 3 \\
\hline -Open Wound - Upper Limb & $880-887$ & 0 & 1 & 1 & 2 \\
\hline -Open Wound - Lower Limb & 890-897 & 0 & 0 & 0 & 1 \\
\hline -Superficial Injury & 910-919 & 0 & 1 & 0 & 2 \\
\hline -Contusion & 920-924 & 4 & 0 & 0 & 8 \\
\hline -Foreign Body Entering Orifice & 930-939 & 0 & 0 & 1 & 1 \\
\hline -Complications \& Unspecified Injuries & 958-959 & 1 & 1 & 1 & 4 \\
\hline -Unspecified Effects - External Causes & $990-995$ & 0 & 1 & 0 & 2 \\
\hline -Complications of Surgical/Medical Care & 996-999 & 0 & 1 & 0 & 6 \\
\hline
\end{tabular}

(Continued)

*Only those diagnostic categories and gender/job category combinations with at least one occurrence appear in this table. 
Idaho National Laboratory 2010

Absence Data

Appendix I. Number of Diagnoses in Each Diagnostic Category by Gender and Job Category*

\begin{tabular}{|c|c|c|c|c|c|}
\hline & & \multicolumn{4}{|c|}{ Men } \\
\hline & & \multicolumn{4}{|c|}{ Job Category } \\
\hline & & Professional & $\begin{array}{c}\text { Administrative } \\
\text { Support }\end{array}$ & Technical Support & Service \\
\hline Diagnostic Category & ICD-9-CM Code & \multirow[b]{2}{*}{1} & \multirow[b]{2}{*}{0} & \multirow[b]{2}{*}{0} & \multirow[b]{2}{*}{0} \\
\hline $\begin{array}{l}\text { HEALTH STATUS/HEALTH SERVICE } \\
\text { CONTACT }\end{array}$ & V01-V89 & & & & \\
\hline -Examination \& Investigation & V70-V82 & 1 & 0 & 0 & 0 \\
\hline
\end{tabular}

*Only those diagnostic categories and gender/job category combinations with at least one occurrence appear in this table. 
Idaho National Laboratory 2010

Absence Data

Appendix I. Number of Diagnoses in Each Diagnostic Category by Gender and Job Category*

\begin{tabular}{|c|c|c|c|c|c|}
\hline & & \multicolumn{4}{|c|}{ Men } \\
\hline & & \multicolumn{3}{|c|}{ Job Category } & \multirow[b]{2}{*}{ TOTAL } \\
\hline & & Security and Fire & Crafts & Line Operators & \\
\hline Diagnostic Category & ICD-9-CM Code & & & & \\
\hline $\begin{array}{l}\text { HEALTH STATUS/HEALTH SERVICE } \\
\text { CONTACT }\end{array}$ & \begin{tabular}{|l|} 
V01-V89 \\
\end{tabular} & 1 & 3 & 0 & 5 \\
\hline -Health Services Reproduction/Development & V20-V29 & 0 & 1 & 0 & 1 \\
\hline -Examination \& Investigation & V70-V82 & 1 & 2 & 0 & 4 \\
\hline
\end{tabular}

\begin{tabular}{|c|c|c|c|c|c|c|c|c|}
\hline & \multicolumn{8}{|c|}{ Men } \\
\hline & \multicolumn{7}{|c|}{ Job Category } & \multirow[b]{2}{*}{ TOTAL } \\
\hline & Professional & $\begin{array}{c}\text { Administrative } \\
\text { Support }\end{array}$ & $\begin{array}{l}\text { Technical } \\
\text { Support }\end{array}$ & Service & $\begin{array}{c}\text { Security and } \\
\text { Fire }\end{array}$ & Crafts & $\begin{array}{c}\text { Line } \\
\text { Operators }\end{array}$ & \\
\hline Diagnostic Category & \multirow[b]{2}{*}{103} & \multirow[b]{2}{*}{59} & \multirow[b]{2}{*}{28} & \multirow[b]{2}{*}{54} & \multirow[b]{2}{*}{54} & \multirow[b]{2}{*}{92} & \multirow[b]{2}{*}{23} & \multirow[b]{2}{*}{413} \\
\hline Total & & & & & & & & \\
\hline
\end{tabular}

*Only those diagnostic categories and gender/job category combinations with at least one occurrence appear in this table. 
Idaho National Laboratory 2010

Absence Data

Appendix J. Total Number of Calendar Days Absent in Each Diagnostic Category by Gender and Job Category*

\begin{tabular}{|c|c|c|c|c|c|}
\hline & \multicolumn{4}{|c|}{ Women } \\
\hline & & \multicolumn{4}{|c|}{ Job Category } \\
\hline & & Professional & $\begin{array}{c}\text { Administrative } \\
\text { Support }\end{array}$ & Technical Support & Service \\
\hline Diagnostic Category & ICD-9-CM Code & \multirow[b]{2}{*}{0} & \multirow[b]{2}{*}{139} & \multirow[b]{2}{*}{0} & \multirow[b]{2}{*}{0} \\
\hline INFECTIOUS \& PARASITIC DISEASES (DIS) & 001-139 & & & & \\
\hline BENIGN \& UNCERTAIN NEOPLASMS & \begin{tabular}{|l|}
$209.4-209.6$ \\
$210-229,235-239$
\end{tabular} & 61 & 0 & 0 & 0 \\
\hline ENDOCRINE/METABOLIC/IMMUNITY & 240-279 & 0 & 0 & 32 & 0 \\
\hline MENTAL DISORDERS & $290-319$ & 0 & 27 & 58 & 0 \\
\hline NERVOUS SYSTEM (NS) \& SENSE ORGANS & $320-389$ & 0 & 57 & 0 & 43 \\
\hline CIRCULATORY SYSTEM & 390-459 & 26 & 23 & 0 & 0 \\
\hline RESPIRATORY SYSTEM & $460-519$ & 31 & 45 & 37 & 19 \\
\hline DIGESTIVE SYSTEM & $520-579$ & 25 & 105 & 34 & 34 \\
\hline GENITOURINARY SYSTEM & $580-629$ & 0 & 177 & 49 & 0 \\
\hline $\begin{array}{l}\text { MUSCULOSKELETAL \& CONNECTIVE } \\
\text { TISSUE }\end{array}$ & 710-739 & 294 & 958 & 0 & 634 \\
\hline $\begin{array}{l}\text { SYMPTOMS, SIGNS, \& ILL-DEFINED } \\
\text { CONDITIONS }\end{array}$ & 780-799 & 2 & 22 & 13 & 10 \\
\hline INJURY \& POISONING & $800-999$ & 729 & 917 & 66 & 355 \\
\hline
\end{tabular}

\begin{tabular}{|c|c|c|c|c|c|}
\hline & & \multicolumn{4}{|c|}{ Women } \\
\hline & & \multicolumn{3}{|c|}{ Job Category } & \multirow[b]{2}{*}{ TOTAL } \\
\hline & & Security and Fire & Crafts & Line Operators & \\
\hline Diagnostic Category & ICD-9-CM Code & \multirow[b]{2}{*}{0} & \multirow[b]{2}{*}{0} & \multirow[b]{2}{*}{0} & \multirow[b]{2}{*}{139} \\
\hline INFECTIOUS \& PARASITIC DISEASES (DIS) & 001-139 & & & & \\
\hline BENIGN \& UNCERTAIN NEOPLASMS & \begin{tabular}{|l|}
$209.4-209.6$ \\
$210-229,235-239$
\end{tabular} & 0 & 178 & 0 & 239 \\
\hline ENDOCRINE/METABOLIC/IMMUNITY & 240-279 & 0 & 0 & 0 & 32 \\
\hline MENTAL DISORDERS & $290-319$ & 0 & 0 & 0 & 85 \\
\hline NERVOUS SYSTEM (NS) \& SENSE ORGANS & $320-389$ & 0 & 0 & 0 & 100 \\
\hline CIRCULATORY SYSTEM & 390-459 & 13 & 0 & 0 & 62 \\
\hline RESPIRATORY SYSTEM & $460-519$ & 72 & 14 & 0 & 218 \\
\hline DIGESTIVE SYSTEM & $520-579$ & 0 & 55 & 0 & 253 \\
\hline GENITOURINARY SYSTEM & $580-629$ & 20 & 11 & 0 & 257 \\
\hline $\begin{array}{l}\text { MUSCULOSKELETAL \& CONNECTIVE } \\
\text { TISSUE }\end{array}$ & $710-739$ & 122 & 230 & 55 & 2,293 \\
\hline $\begin{array}{l}\text { SYMPTOMS, SIGNS, \& ILL-DEFINED } \\
\text { CONDITIONS }\end{array}$ & $780-799$ & 0 & 0 & 0 & 47 \\
\hline INJURY \& POISONING & $800-999$ & 0 & 40 & 0 & 2,107 \\
\hline
\end{tabular}

\footnotetext{
*Absences with >1 ICD-9-CM code in the same diagnostic category were counted only once. Only those diagnostic categories and gender/job category combinations with at least one occurrence appear in this table.
} 
Idaho National Laboratory 2010

Absence Data

Appendix J. Total Number of Calendar Days Absent in Each Diagnostic Category by Gender and Job Category*

\begin{tabular}{|c|c|c|c|c|c|}
\hline & \multicolumn{4}{|c|}{ Men } \\
\hline & & \multicolumn{4}{|c|}{ Job Category } \\
\hline & & Professional & $\begin{array}{c}\text { Administrative } \\
\text { Support }\end{array}$ & Technical Support & Service \\
\hline Diagnostic Category & ICD-9-CM Code & \multirow[b]{2}{*}{32} & \multirow[b]{2}{*}{0} & \multirow[b]{2}{*}{0} & \multirow[b]{2}{*}{0} \\
\hline INFECTIOUS \& PARASITIC DISEASES (DIS) & 001-139 & & & & \\
\hline MALIGNANT NEOPLASMS & \begin{tabular}{|l|}
$140-208$ \\
$209.0-209.3$ \\
$230-234$
\end{tabular} & 502 & 85 & 0 & 228 \\
\hline BENIGN \& UNCERTAIN NEOPLASMS & \begin{tabular}{|l|}
$209.4-209.6$ \\
$210-229$, \\
\end{tabular} & 20 & 0 & 0 & 0 \\
\hline ENDOCRINE/METABOLIC/IMMUNITY & 240-279 & 25 & 2 & 0 & 0 \\
\hline MENTAL DISORDERS & $290-319$ & 1 & 0 & 0 & 9 \\
\hline NERVOUS SYSTEM (NS) \& SENSE ORGANS & $320-389$ & 39 & 363 & 92 & 0 \\
\hline CIRCULATORY SYSTEM & $390-459$ & 148 & 67 & 3 & 211 \\
\hline RESPIRATORY SYSTEM & $460-519$ & 133 & 337 & 15 & 18 \\
\hline DIGESTIVE SYSTEM & $520-579$ & 309 & 134 & 1,206 & 137 \\
\hline GENITOURINARY SYSTEM & $580-629$ & 0 & 21 & 0 & 134 \\
\hline SKIN AND SUBCUTANEOUS TISSUE & 680-709 & 0 & 11 & 0 & 0 \\
\hline $\begin{array}{l}\text { MUSCULOSKELETAL \& CONNECTIVE } \\
\text { TISSUE }\end{array}$ & $710-739$ & 1,069 & 242 & 306 & 1,281 \\
\hline $\begin{array}{l}\text { SYMPTOMS, SIGNS, \& ILL-DEFINED } \\
\text { CONDITIONS }\end{array}$ & 780-799 & 6 & 74 & 0 & 12 \\
\hline INJURY \& POISONING & $800-999$ & 703 & 939 & 776 & 570 \\
\hline
\end{tabular}

\footnotetext{
*Absences with >1 ICD-9-CM code in the same diagnostic category were counted only once. Only those diagnostic categories and gender/job category combinations with at least one occurrence appear in this table.
} 
Idaho National Laboratory 2010

Absence Data

Appendix J. Total Number of Calendar Days Absent in Each Diagnostic Category by Gender and Job Category*

\begin{tabular}{|c|c|c|c|c|c|}
\hline & & \multicolumn{4}{|c|}{ Men } \\
\hline & & \multicolumn{3}{|c|}{ Job Category } & \multirow[b]{2}{*}{ TOTAL } \\
\hline & & Security and Fire & Crafts & Line Operators & \\
\hline Diagnostic Category & ICD-9-CM Code & \multirow[b]{2}{*}{0} & \multirow[b]{2}{*}{0} & \multirow[b]{2}{*}{14} & \multirow[b]{2}{*}{46} \\
\hline INFECTIOUS \& PARASITIC DISEASES (DIS) & 001-139 & & & & \\
\hline MALIGNANT NEOPLASMS & $\begin{array}{l}140-208 \\
209.0-209.3 \\
230-234\end{array}$ & 0 & 55 & 0 & 870 \\
\hline BENIGN \& UNCERTAIN NEOPLASMS & $\begin{array}{l}209.4-209.6 \\
210-229,235-239\end{array}$ & 0 & 0 & 0 & 20 \\
\hline ENDOCRINE/METABOLIC/IMMUNITY & $240-279$ & 6 & 72 & 5 & 110 \\
\hline MENTAL DISORDERS & 290-319 & 0 & 0 & 0 & 10 \\
\hline NERVOUS SYSTEM (NS) \& SENSE ORGANS & 320-389 & 38 & 182 & 8 & 722 \\
\hline CIRCULATORY SYSTEM & $390-459$ & 15 & 105 & 102 & 651 \\
\hline RESPIRATORY SYSTEM & $460-519$ & 166 & 145 & 14 & 828 \\
\hline DIGESTIVE SYSTEM & $520-579$ & 237 & 64 & 18 & 2,105 \\
\hline GENITOURINARY SYSTEM & $580-629$ & 32 & 65 & 0 & 252 \\
\hline SKIN AND SUBCUTANEOUS TISSUE & 680-709 & 0 & 39 & 0 & 50 \\
\hline $\begin{array}{l}\text { MUSCULOSKELETAL \& CONNECTIVE } \\
\text { TISSUE }\end{array}$ & 710-739 & 599 & 1,034 & 582 & 5,113 \\
\hline $\begin{array}{l}\text { SYMPTOMS, SIGNS, \& ILL-DEFINED } \\
\text { CONDITIONS }\end{array}$ & 780-799 & 21 & 31 & 0 & 144 \\
\hline INJURY \& POISONING & 800-999 & 844 & 2,095 & 271 & 6,198 \\
\hline
\end{tabular}

\footnotetext{
*Absences with >1 ICD-9-CM code in the same diagnostic category were counted only once. Only those diagnostic categories and gender/job category combinations with at least one occurrence appear in this table.
} 
Idaho National Laboratory 2010

Absence Data

Appendix K. Age-Adjusted Illness and Injury Rates by Diagnostic Category*

Part 1. Men

\begin{tabular}{|c|c|c|c|c|c|}
\hline & & $\begin{array}{l}\text { Number of } \\
\text { Diagnoses }\end{array}$ & $\begin{array}{c}\text { Age-Adjusted } \\
\text { Rate per } 1,000 * *\end{array}$ & $\begin{array}{c}\text { Lower } 95 \% \\
\text { Confidence } \\
\text { Limit per 1,000 }\end{array}$ & $\begin{array}{c}\text { Upper } 95 \% \\
\text { Confidence } \\
\text { Limit per 1,000 }\end{array}$ \\
\hline Diagnostic Category & ICD-9-CM Code & & & & \\
\hline INFECTIOUS \& PARASITIC DISEASES (DIS) & 001-139 & 2 & 1.4 & 0.3 & 6.2 \\
\hline -Other Bacterial Dis & 030-041 & 2 & 1.4 & 0.3 & 6.2 \\
\hline MALIGNANT NEOPLASMS & $\begin{array}{l}140-208, \\
209.0-209.3 \\
230-234\end{array}$ & 10 & 1.8 & 1.0 & 3.5 \\
\hline -Digestive \& Peritoneal & $150-159$ & 2 & 0.2 & 0.1 & 1.0 \\
\hline -Bone, Connective Tissue, Skin & $170-173,176$ & 1 & 0.1 & 0.0 & 0.8 \\
\hline -Genitourinary & $179-189$ & 4 & 0.8 & 0.3 & 2.2 \\
\hline -Lymphatic \& Hematopoietic & $200-208$ & 3 & 0.6 & 0.2 & 2.0 \\
\hline BENIGN \& UNCERTAIN NEOPLASMS & \begin{tabular}{|l|}
$209.4-209.6$ \\
$210-229$, \\
$235-239$
\end{tabular} & 1 & 0.1 & 0.0 & 0.8 \\
\hline ENDOCRINE/METABOLIC/IMMUNITY & $240-279$ & 7 & 1.8 & 0.8 & 4.0 \\
\hline -Other Endocrine Gland Dis & 249-259 & 2 & 0.4 & 0.1 & 1.8 \\
\hline -Other Metabolic \& Immunity Disorders & $270-279$ & 5 & 1.4 & 0.5 & 3.6 \\
\hline MENTAL DISORDERS & 290-319 & 2 & 0.2 & 0.1 & 1.0 \\
\hline -Non-Psychotic Disorders & $300-302,306-316$ & 2 & 0.2 & 0.1 & 1.0 \\
\hline NERVOUS SYSTEM (NS) \& SENSE ORGANS & $320-389$ & 21 & 4.9 & 2.7 & 9.2 \\
\hline -Other Disorders of Central NS & 340-349 & 4 & 2.3 & 0.7 & 7.6 \\
\hline -Disorders of Peripheral NS & $350-359$ & 3 & 0.5 & 0.2 & 1.7 \\
\hline -Disorders of Eye & $360-379$ & 8 & 1.3 & 0.6 & 2.7 \\
\hline -Diseases of Ear \& Mastoid & $380-389$ & 6 & 0.8 & 0.4 & 1.8 \\
\hline CIRCULATORY SYSTEM & $390-459$ & 31 & 8.0 & 5.1 & 12.6 \\
\hline -Hypertensive Dis & $401-405$ & 8 & 1.4 & 0.7 & 3.2 \\
\hline -Ischemic Heart Dis & $410-414$ & 9 & 1.4 & 0.7 & 2.8 \\
\hline -Dis of Pulmonary Circulation & $415-417$ & 4 & 1.0 & 0.3 & 2.8 \\
\hline -Other Heart Dis & $420-429$ & 6 & 3.4 & 1.4 & 8.4 \\
\hline -Cerebrovascular Dis & $430-438$ & 2 & 0.4 & 0.1 & 1.7 \\
\hline -Dis of Veins, Lymphatics, Other & 451-459 & 2 & 0.3 & 0.1 & 1.4 \\
\hline RESPIRATORY SYSTEM & $460-519$ & 41 & 10.9 & 7.4 & 15.9 \\
\hline -Acute Respiratory Infections & $460-466$ & 4 & 1.6 & 0.4 & 6.0 \\
\hline -Other Dis Upper Respiratory Tract & $470-478$ & 26 & 6.8 & 4.3 & 10.8 \\
\hline -Pneumonia \& Influenza & $480-488$ & 3 & 0.7 & 0.2 & 2.5 \\
\hline -Chronic Obstructive Dis & $490-496$ & 7 & 1.6 & 0.7 & 3.5 \\
\hline -Lung Dis from External Agents & $500-508$ & 1 & 0.2 & 0.0 & 1.6 \\
\hline DIGESTIVE SYSTEM & $520-579$ & 45 & 11.3 & 7.9 & 16.3 \\
\hline -Oral Cavity, Saliva Glands, Jaw & $520-529$ & 3 & 0.8 & 0.3 & 2.8 \\
\hline
\end{tabular}

(Continued)

* Only those diagnostic categories with at least one occurrence appear in this table.

**Standardized to age distribution of 2000 U.S. population. 
Idaho National Laboratory 2010

Absence Data

Appendix K. Age-Adjusted Illness and Injury Rates by Diagnostic Category*

Part 1. Men

\begin{tabular}{|c|c|c|c|c|c|}
\hline & & $\begin{array}{l}\text { Number of } \\
\text { Diagnoses }\end{array}$ & $\begin{array}{c}\text { Age-Adjusted } \\
\text { Rate per } 1,000 * *\end{array}$ & $\begin{array}{c}\text { Lower } 95 \% \\
\text { Confidence } \\
\text { Limit per } 1,000\end{array}$ & $\begin{array}{c}\text { Upper 95\% } \\
\text { Confidence } \\
\text { Limit per 1,000 }\end{array}$ \\
\hline Diagnostic Category & ICD-9-CM Code & \multirow[b]{2}{*}{5} & \multirow[b]{2}{*}{2.0} & \multirow[b]{2}{*}{0.6} & \multirow[b]{2}{*}{6.0} \\
\hline -Esophagus, Stomach, Duodenum & $530-538$ & & & & \\
\hline -Appendicitis & $540-543$ & 2 & 1.2 & 0.2 & 6.2 \\
\hline -Hernias & $550-553$ & 19 & 3.9 & 2.4 & 6.5 \\
\hline -Enteritis, Colitis & $555-558$ & 3 & 0.7 & 0.2 & 2.5 \\
\hline -Other Intestinal Dis & $560-569$ & 1 & 0.2 & 0.0 & $\overline{1.6}$ \\
\hline -Other Digestive Dis & $570-579$ & 12 & 2.5 & 1.4 & 4.6 \\
\hline GENITOURINARY SYSTEM & $580-629$ & 8 & 2.4 & 0.9 & 6.1 \\
\hline -Other Urinary Dis & $590-599$ & 2 & 0.6 & 0.1 & 2.7 \\
\hline -Male Genital Organ Dis & $600-608$ & 6 & 1.8 & 0.5 & 5.6 \\
\hline SKIN AND SUBCUTANEOUS TISSUE & $680-709$ & 2 & 1.2 & 0.2 & 6.2 \\
\hline -Infections & $680-686$ & 2 & 1.2 & 0.2 & 6.2 \\
\hline $\begin{array}{l}\text { MUSCULOSKELETAL \& CONNECTIVE } \\
\text { TISSUE }\end{array}$ & 710-739 & 89 & 18.2 & 14.1 & 23.4 \\
\hline -Arthropathies & $710-719$ & 43 & 8.2 & 5.9 & 11.3 \\
\hline -Dorsopathies & $720-724$ & 33 & 7.8 & 5.0 & 12.2 \\
\hline -Rheumatism, Excluding Back & $725-729$ & 10 & 1.8 & 0.9 & 3.6 \\
\hline -Other Dis \& Acquired Deformities & 730-739 & 3 & 0.5 & 0.1 & 1.5 \\
\hline $\begin{array}{l}\text { SYMPTOMS, SIGNS, \& ILL-DEFINED } \\
\text { CONDITIONS }\end{array}$ & $780-799$ & 13 & 2.5 & 1.4 & 4.5 \\
\hline -Symptoms & 780-789 & 12 & 2.4 & 1.3 & 4.4 \\
\hline -Non-Specific Abnormal Findings & $790-796$ & 1 & 0.1 & 0.0 & 0.8 \\
\hline INJURY \& POISONING & $800-999$ & 136 & 34.5 & 27.8 & 42.8 \\
\hline -Fracture - Skull & 800-804 & 4 & 0.9 & 0.3 & 2.6 \\
\hline -Fracture - Upper Limb & $810-819$ & 11 & 3.6 & 1.6 & 8.2 \\
\hline -Fracture - Lower Limb & 820-829 & 7 & 1.7 & 0.8 & 3.9 \\
\hline -Dislocation & $830-839$ & 6 & 2.8 & 1.0 & 7.8 \\
\hline -Sprains \& Strains - Back & 846-847 & 15 & 4.1 & 2.2 & 7.9 \\
\hline -Sprains \& Strains - Other & $840-845,848$ & 63 & 13.6 & 10.1 & 18.1 \\
\hline -Intracranial Injury & $850-854$ & 1 & 0.1 & 0.0 & 0.8 \\
\hline -Open Wound - Head, Neck, Trunk & $870-879$ & 6 & 1.5 & 0.6 & 3.7 \\
\hline -Superficial Injury & 910-919 & 2 & 1.1 & 0.2 & 6.4 \\
\hline -Contusion & 920-924 & 8 & 1.3 & 0.6 & 2.7 \\
\hline -Foreign Body Entering Orifice & 930-939 & 1 & 0.1 & 0.0 & 0.8 \\
\hline -Complications \& Unspecified Injuries & 958-959 & 4 & 0.9 & 0.3 & 2.7 \\
\hline -Unspecified Effects - External Causes & $990-995$ & 2 & 1.2 & 0.2 & 6.2 \\
\hline
\end{tabular}

(Continued)

* Only those diagnostic categories with at least one occurrence appear in this table.

**Standardized to age distribution of 2000 U.S. population. 
Idaho National Laboratory 2010

Absence Data

Appendix K. Age-Adjusted IIIness and Injury Rates by Diagnostic Category*

Part 1. Men

\begin{tabular}{|c|c|c|c|c|c|}
\hline & & $\begin{array}{l}\text { Number of } \\
\text { Diagnoses }\end{array}$ & $\begin{array}{c}\text { Age-Adjusted } \\
\text { Rate per } 1,000^{* *}\end{array}$ & $\begin{array}{c}\text { Lower } 95 \% \\
\text { Confidence } \\
\text { Limit per } 1,000\end{array}$ & $\begin{array}{c}\text { Upper } 95 \% \\
\text { Confidence } \\
\text { Limit per 1,000 }\end{array}$ \\
\hline Diagnostic Category & ICD-9-CM Code & \multirow[b]{2}{*}{6} & \multirow[b]{2}{*}{1.5} & \multirow[b]{2}{*}{0.7} & \multirow[b]{2}{*}{3.5} \\
\hline -Complications of Surgical/Medical Care & 996-999 & & & & \\
\hline Total & & 408 & 99.4 & 87.8 & 112.5 \\
\hline
\end{tabular}

* Only those diagnostic categories with at least one occurrence appear in this table.

**Standardized to age distribution of 2000 U.S. population. 
Idaho National Laboratory 2010

Absence Data

Appendix K. Age-Adjusted Illness and Injury Rates by Diagnostic Category*

Part 2. Women

\begin{tabular}{|c|c|c|c|c|c|}
\hline & & $\begin{array}{l}\text { Number of } \\
\text { Diagnoses }\end{array}$ & $\begin{array}{c}\text { Age-Adjusted } \\
\text { Rate per } 1,000 * *\end{array}$ & $\begin{array}{c}\text { Lower } 95 \% \\
\text { Confidence } \\
\text { Limit per 1,000 }\end{array}$ & $\begin{array}{c}\text { Upper } 95 \% \\
\text { Confidence } \\
\text { Limit per 1,000 }\end{array}$ \\
\hline Diagnostic Category & ICD-9-CM Code & & & & \\
\hline INFECTIOUS \& PARASITIC DISEASES (DIS) & 001-139 & 4 & 1.5 & 0.6 & 4.1 \\
\hline -Intestinal Infectious Dis & 001-009 & 1 & 0.4 & 0.1 & 2.7 \\
\hline -Syphilis \& Other Venereal Dis & 090-099 & 2 & 0.8 & 0.2 & 3.1 \\
\hline -Mycoses & 110-118 & 1 & 0.4 & 0.1 & 2.7 \\
\hline BENIGN \& UNCERTAIN NEOPLASMS & \begin{tabular}{|l|}
$209.4-209.6$ \\
$210-229$, \\
$235-239$
\end{tabular} & 2 & 0.8 & 0.2 & 3.1 \\
\hline ENDOCRINE/METABOLIC/IMMUNITY & $240-279$ & 1 & 2.3 & 0.3 & 16.5 \\
\hline -Other Metabolic \& Immunity Disorders & $270-279$ & 1 & 2.3 & 0.3 & 16.5 \\
\hline MENTAL DISORDERS & $290-319$ & 3 & 3.5 & 0.9 & 13.9 \\
\hline -Non-Psychotic Disorders & $300-302,306-316$ & 3 & 3.5 & 0.9 & 13.9 \\
\hline NERVOUS SYSTEM (NS) \& SENSE ORGANS & $320-389$ & 4 & 2.6 & 0.9 & 8.1 \\
\hline -Other Disorders of Central NS & $340-349$ & 1 & 1.3 & 0.2 & 9.1 \\
\hline -Disorders of Peripheral NS & $350-359$ & 3 & 1.4 & 0.4 & 4.3 \\
\hline CIRCULATORY SYSTEM & $390-459$ & 4 & 2.7 & 1.0 & 7.7 \\
\hline -Ischemic Heart Dis & $410-414$ & 1 & 0.4 & 0.1 & 2.7 \\
\hline -Dis of Pulmonary Circulation & $415-417$ & 1 & 0.9 & 0.1 & 6.3 \\
\hline -Cerebrovascular Dis & $430-438$ & 1 & 0.9 & 0.1 & 6.3 \\
\hline -Dis of Veins, Lymphatics, Other & $451-459$ & 1 & 0.6 & 0.1 & 4.1 \\
\hline RESPIRATORY SYSTEM & $460-519$ & 13 & 8.7 & 4.4 & 17.3 \\
\hline -Other Dis Upper Respiratory Tract & $470-478$ & 3 & 1.4 & 0.4 & 4.3 \\
\hline -Pneumonia \& Influenza & $480-488$ & 4 & 4.0 & 1.1 & 14.0 \\
\hline -Chronic Obstructive Dis & $490-496$ & 3 & 1.9 & 0.6 & 6.1 \\
\hline -Other Respiratory Dis & $510-519$ & 3 & 1.5 & 0.5 & 4.9 \\
\hline DIGESTIVE SYSTEM & $520-579$ & 16 & 11.9 & 6.3 & 22.7 \\
\hline -Oral Cavity, Saliva Glands, Jaw & $520-529$ & 1 & 0.4 & 0.1 & 2.7 \\
\hline -Esophagus, Stomach, Duodenum & \begin{tabular}{|l|}
$530-538$ \\
\end{tabular} & 2 & 1.0 & 0.2 & 4.0 \\
\hline -Hernias & $550-553$ & 5 & 4.1 & 1.2 & 13.5 \\
\hline -Enteritis, Colitis & $555-558$ & 1 & 2.3 & 0.3 & 16.5 \\
\hline -Other Intestinal Dis & $560-569$ & 1 & 0.4 & 0.1 & 2.7 \\
\hline -Other Digestive Dis & \begin{tabular}{|l|}
$570-579$ \\
\end{tabular} & 6 & 3.8 & 1.6 & 9.2 \\
\hline GENITOURINARY SYSTEM & $580-629$ & 12 & 8.7 & 4.8 & 15.8 \\
\hline -Other Urinary Dis & $590-599$ & 2 & 1.0 & 0.2 & 4.0 \\
\hline -Breast Disorders & $610-612$ & 2 & 1.5 & 0.4 & 6.1 \\
\hline -Pelvic Inflammatory Dis & 614-616 & 1 & 0.6 & 0.1 & 4.1 \\
\hline -Other Female Disorders & $617-629$ & 7 & 5.7 & 2.6 & 12.3 \\
\hline
\end{tabular}

(Continued)

*Only those diagnostic categories with at least one occurrence appear in this table.

**Standardized to age distribution of 2000 U.S. population. 
Idaho National Laboratory 2010

Absence Data

Appendix K. Age-Adjusted Illness and Injury Rates by Diagnostic Category*

Part 2. Women

\begin{tabular}{|c|c|c|c|c|c|}
\hline & & $\begin{array}{l}\text { Number of } \\
\text { Diagnoses }\end{array}$ & $\begin{array}{c}\text { Age-Adjusted } \\
\text { Rate per } 1,000 * *\end{array}$ & \begin{tabular}{|c|} 
Lower $95 \%$ \\
Confidence \\
Limit per 1,000
\end{tabular} & $\begin{array}{c}\text { Upper } 95 \% \\
\text { Confidence } \\
\text { Limit per 1,000 }\end{array}$ \\
\hline Diagnostic Category & ICD-9-CM Code & & & & \\
\hline $\begin{array}{l}\text { MUSCULOSKELETAL \& CONNECTIVE } \\
\text { TISSUE }\end{array}$ & $710-739$ & 47 & 32.7 & 23.0 & 46.6 \\
\hline -Arthropathies & 710-719 & 21 & 11.9 & 7.4 & 19.1 \\
\hline -Dorsopathies & $720-724$ & 10 & 7.1 & 3.2 & 15.6 \\
\hline -Rheumatism, Excluding Back & 725-729 & 15 & 13.2 & 7.0 & 24.7 \\
\hline -Other Dis \& Acquired Deformities & 730-739 & 1 & 0.6 & 0.1 & 4.1 \\
\hline $\begin{array}{l}\text { SYMPTOMS, SIGNS, \& ILL-DEFINED } \\
\text { CONDITIONS }\end{array}$ & $780-799$ & 9 & 9.5 & 4.0 & 22.4 \\
\hline -Symptoms & 780-789 & 7 & 6.8 & 2.5 & 18.3 \\
\hline -Non-Specific Abnormal Findings & $790-796$ & 2 & 2.7 & 0.5 & 14.9 \\
\hline INJURY \& POISONING & $800-999$ & 59 & 38.7 & 28.2 & 53.1 \\
\hline -Fracture - Skull & $800-804$ & 3 & 7.0 & 2.3 & 21.6 \\
\hline -Fracture - Upper Limb & $810-819$ & 3 & 1.4 & 0.4 & 4.3 \\
\hline -Fracture - Lower Limb & 820-829 & 2 & 1.5 & 0.4 & 6.1 \\
\hline -Dislocation & 830-839 & 8 & 4.8 & 2.2 & 10.2 \\
\hline -Sprains \& Strains - Back & 846-847 & 10 & 7.5 & 3.5 & 16.0 \\
\hline -Sprains \& Strains - Other & $840-845,848$ & 20 & 10.0 & 6.3 & 15.7 \\
\hline -Open Wound - Head, Neck, Trunk & 870-879 & 1 & 0.6 & 0.1 & 4.1 \\
\hline -Superficial Injury & 910-919 & 1 & 0.4 & 0.1 & 2.7 \\
\hline -Contusion & $920-924$ & 8 & 4.4 & 2.2 & 8.9 \\
\hline -Complications \& Unspecified Injuries & 958-959 & 2 & 1.0 & 0.2 & 4.0 \\
\hline -Toxic Effects - Non-medicinal & $980-989$ & 1 & 0.4 & 0.1 & 2.7 \\
\hline Total & & 174 & 123.8 & 102.7 & 149.2 \\
\hline
\end{tabular}

* Only those diagnostic categories with at least one occurrence appear in this table.

**Standardized to age distribution of 2000 U.S. population. 
Idaho National Laboratory 2010

Absence Data

Appendix K. Age-Adjusted Illness and Injury Rates by Diagnostic Category*

Part 3. Men and Women

\begin{tabular}{|c|c|c|c|c|c|}
\hline & & $\begin{array}{l}\text { Number of } \\
\text { Diagnoses }\end{array}$ & $\begin{array}{c}\text { Age-Adjusted } \\
\text { Rate per 1,000** }\end{array}$ & \begin{tabular}{|c|} 
Lower $95 \%$ \\
Confidence \\
Limit per 1,000
\end{tabular} & $\begin{array}{c}\text { Upper } 95 \% \\
\text { Confidence } \\
\text { Limit per } 1,000\end{array}$ \\
\hline Diagnostic Category & ICD-9-CM Code & & & & \\
\hline INFECTIOUS \& PARASITIC DISEASES (DIS) & 001-139 & 6 & 1.4 & 0.4 & 4.1 \\
\hline -Intestinal Infectious Dis & 001-009 & 1 & 0.1 & 0.0 & 0.7 \\
\hline -Other Bacterial Dis & 030-041 & 2 & 1.0 & 0.2 & 4.4 \\
\hline -Syphilis \& Other Venereal Dis & 090-099 & 2 & 0.2 & 0.0 & 0.7 \\
\hline -Mycoses & 110-118 & 1 & 0.1 & 0.0 & 0.7 \\
\hline MALIGNANT NEOPLASMS & $\begin{array}{l}140-208, \\
209.0-209.3, \\
230-234\end{array}$ & 10 & 1.5 & 0.8 & 2.7 \\
\hline -Digestive \& Peritoneal & $150-159$ & 2 & 0.2 & 0.0 & 0.7 \\
\hline -Bone, Connective Tissue, Skin & 170-173, 176 & 1 & 0.1 & 0.0 & 0.7 \\
\hline -Genitourinary & 179-189 & 4 & 0.7 & 0.3 & 1.8 \\
\hline -Lymphatic \& Hematopoietic & 200-208 & 3 & 0.5 & 0.2 & 1.5 \\
\hline BENIGN \& UNCERTAIN NEOPLASMS & $\begin{array}{l}209.4-209.6, \\
210-229,235-239\end{array}$ & 3 & 0.3 & 0.1 & 0.9 \\
\hline ENDOCRINE/METABOLIC/IMMUNITY & $240-279$ & 8 & 2.0 & 0.9 & 4.7 \\
\hline -Other Endocrine Gland Dis & 249-259 & 2 & 0.3 & 0.1 & 1.3 \\
\hline -Other Metabolic \& Immunity Disorders & $270-279$ & 6 & 1.7 & 0.7 & 4.5 \\
\hline MENTAL DISORDERS & 290-319 & 5 & 1.2 & 0.4 & 4.0 \\
\hline -Non-Psychotic Disorders & $300-302,306-316$ & 5 & 1.2 & 0.4 & 4.0 \\
\hline NERVOUS SYSTEM (NS) \& SENSE ORGANS & 320-389 & 25 & 4.4 & 2.6 & 7.5 \\
\hline -Other Disorders of Central NS & 340-349 & 5 & 1.9 & 0.7 & 5.5 \\
\hline -Disorders of Peripheral NS & 350-359 & 6 & 0.8 & 0.3 & 1.8 \\
\hline -Disorders of Eye & $360-379$ & 8 & 1.0 & 0.5 & 2.2 \\
\hline -Diseases of Ear \& Mastoid & 380-389 & 6 & 0.6 & 0.3 & 1.5 \\
\hline CIRCULATORY SYSTEM & $390-459$ & 35 & 6.7 & 4.4 & 10.0 \\
\hline -Hypertensive Dis & $401-405$ & 8 & 1.1 & 0.5 & 2.4 \\
\hline -Ischemic Heart Dis & $410-414$ & 10 & 1.2 & 0.6 & 2.4 \\
\hline -Dis of Pulmonary Circulation & 415-417 & 5 & 0.9 & 0.4 & 2.3 \\
\hline -Other Heart Dis & $420-429$ & 6 & 2.5 & 1.0 & 6.0 \\
\hline -Cerebrovascular Dis & $430-438$ & 3 & 0.5 & 0.2 & 1.6 \\
\hline -Dis of Veins, Lymphatics, Other & $451-459$ & 3 & 0.4 & 0.1 & $\overline{1.3}$ \\
\hline RESPIRATORY SYSTEM & $460-519$ & 54 & 10.4 & 7.5 & 14.5 \\
\hline -Acute Respiratory Infections & $460-466$ & 4 & 1.2 & 0.3 & 4.2 \\
\hline -Other Dis Upper Respiratory Tract & $470-478$ & 29 & 5.4 & 3.5 & 8.4 \\
\hline -Pneumonia \& Influenza & $480-488$ & 7 & 1.5 & 0.6 & 4.2 \\
\hline -Chronic Obstructive Dis & $490-496$ & 10 & 1.7 & 0.9 & 3.2 \\
\hline
\end{tabular}

(Continued)

* Only those diagnostic categories with at least one occurrence appear in this table.

**Standardized to age distribution of 2000 U.S. population. 
Idaho National Laboratory 2010

Absence Data

Appendix K. Age-Adjusted Illness and Injury Rates by Diagnostic Category*

Part 3. Men and Women

\begin{tabular}{|c|c|c|c|c|c|}
\hline & & $\begin{array}{l}\text { Number of } \\
\text { Diagnoses }\end{array}$ & $\begin{array}{c}\text { Age-Adjusted } \\
\text { Rate per } 1,000 * *\end{array}$ & $\begin{array}{c}\text { Lower } 95 \% \\
\text { Confidence } \\
\text { Limit per 1,000 }\end{array}$ & $\begin{array}{c}\text { Upper 95\% } \\
\text { Confidence } \\
\text { Limit per 1,000 }\end{array}$ \\
\hline Diagnostic Category & ICD-9-CM Code & \multirow[b]{2}{*}{1} & \multirow[b]{2}{*}{0.2} & \multirow[b]{2}{*}{0.0} & \multirow[b]{2}{*}{1.1} \\
\hline -Lung Dis from External Agents & $500-508$ & & & & \\
\hline -Other Respiratory Dis & $510-519$ & 3 & 0.4 & 0.1 & 1.3 \\
\hline DIGESTIVE SYSTEM & $520-579$ & 61 & 11.6 & 8.4 & 15.9 \\
\hline -Oral Cavity, Saliva Glands, Jaw & $520-529$ & 4 & 0.7 & 0.3 & 2.1 \\
\hline -Esophagus, Stomach, Duodenum & $530-538$ & 7 & 1.7 & 0.6 & 4.3 \\
\hline -Appendicitis & $540-543$ & 2 & 0.8 & 0.2 & 4.3 \\
\hline -Hernias & $550-553$ & 24 & 4.1 & 2.5 & 6.7 \\
\hline -Enteritis, Colitis & $555-558$ & 4 & 1.2 & 0.3 & 4.2 \\
\hline -Other Intestinal Dis & $560-569$ & 2 & 0.3 & 0.1 & 1.1 \\
\hline -Other Digestive Dis & $570-579$ & 18 & 2.8 & 1.7 & 4.7 \\
\hline GENITOURINARY SYSTEM & $580-629$ & 20 & 3.8 & 2.3 & 6.5 \\
\hline -Other Urinary Dis & $590-599$ & 4 & 0.7 & 0.2 & 2.1 \\
\hline -Male Genital Organ Dis & $600-608$ & 6 & 1.3 & 0.4 & 4.0 \\
\hline -Breast Disorders & 610-612 & 2 & 0.3 & 0.1 & $\overline{1.3}$ \\
\hline -Pelvic Inflammatory Dis & 614-616 & 1 & 0.2 & 0.0 & 1.1 \\
\hline -Other Female Disorders & $617-629$ & 7 & 1.3 & 0.6 & 2.8 \\
\hline SKIN AND SUBCUTANEOUS TISSUE & 680-709 & 2 & 0.9 & 0.2 & 4.3 \\
\hline -Infections & $680-686$ & 2 & 0.9 & 0.2 & 4.3 \\
\hline $\begin{array}{l}\text { MUSCULOSKELETAL \& CONNECTIVE } \\
\text { TISSUE }\end{array}$ & $710-739$ & 136 & 21.9 & 17.8 & 26.9 \\
\hline -Arthropathies & $710-719$ & 64 & 9.0 & 6.9 & 11.8 \\
\hline -Dorsopathies & $720-724$ & 43 & 7.7 & 5.2 & 11.3 \\
\hline -Rheumatism, Excluding Back & $725-729$ & 25 & 4.7 & 2.8 & 7.8 \\
\hline -Other Dis \& Acquired Deformities & $730-739$ & 4 & 0.5 & 0.2 & 1.4 \\
\hline $\begin{array}{l}\text { SYMPTOMS, SIGNS, \& ILL-DEFINED } \\
\text { CONDITIONS }\end{array}$ & $780-799$ & 22 & 4.6 & 2.6 & 8.1 \\
\hline -Symptoms & 780-789 & 19 & 3.7 & 2.0 & 6.8 \\
\hline -Non-Specific Abnormal Findings & $790-796$ & 3 & 0.9 & 0.2 & 4.2 \\
\hline INJURY \& POISONING & 800-999 & 195 & 35.9 & 30.1 & 42.9 \\
\hline -Fracture - Skull & $800-804$ & 7 & 2.7 & 1.1 & 6.7 \\
\hline -Fracture - Upper Limb & 810-819 & 14 & 3.0 & 1.4 & 6.1 \\
\hline -Fracture - Lower Limb & $820-829$ & 9 & 1.6 & 0.8 & 3.3 \\
\hline -Dislocation & 830-839 & 14 & 3.2 & 1.6 & 6.4 \\
\hline -Sprains \& Strains - Back & $846-847$ & 25 & 5.0 & 3.1 & 8.3 \\
\hline -Sprains \& Strains - Other & $840-845,848$ & 83 & 12.7 & 10.0 & 16.3 \\
\hline -Intracranial Injury & $850-854$ & 1 & 0.1 & 0.0 & 0.7 \\
\hline
\end{tabular}

(Continued)

* Only those diagnostic categories with at least one occurrence appear in this table.

**Standardized to age distribution of 2000 U.S. population. 
Idaho National Laboratory 2010

Absence Data

Appendix K. Age-Adjusted Illness and Injury Rates by Diagnostic Category*

Part 3. Men and Women

\begin{tabular}{|c|c|c|c|c|c|}
\hline & & $\begin{array}{l}\text { Number of } \\
\text { Diagnoses }\end{array}$ & $\begin{array}{c}\text { Age-Adjusted } \\
\text { Rate per } 1,000 * *\end{array}$ & $\begin{array}{c}\text { Lower } 95 \% \\
\text { Confidence } \\
\text { Limit per 1,000 }\end{array}$ & $\begin{array}{c}\text { Upper } 95 \% \\
\text { Confidence } \\
\text { Limit per 1,000 }\end{array}$ \\
\hline Diagnostic Category & ICD-9-CM Code & \multirow[b]{2}{*}{7} & \multirow[b]{2}{*}{1.3} & \multirow[b]{2}{*}{0.6} & \multirow[b]{2}{*}{2.9} \\
\hline -Open Wound - Head, Neck, Trunk & $870-879$ & & & & \\
\hline -Superficial Injury & $910-919$ & 3 & 0.9 & 0.2 & 4.2 \\
\hline -Contusion & 920-924 & 16 & 2.2 & 1.4 & 3.7 \\
\hline -Foreign Body Entering Orifice & 930-939 & 1 & 0.1 & 0.0 & 0.7 \\
\hline -Complications \& Unspecified Injuries & 958-959 & 6 & 0.9 & 0.4 & 2.2 \\
\hline -Toxic Effects - Non-medicinal & $980-989$ & 1 & 0.1 & 0.0 & 0.7 \\
\hline -Unspecified Effects - External Causes & $990-995$ & 2 & 0.8 & 0.2 & 4.3 \\
\hline -Complications of Surgical/Medical Care & 996-999 & 6 & 1.1 & 0.5 & 2.6 \\
\hline Total & & 582 & 106.5 & 96.0 & 118.0 \\
\hline
\end{tabular}

* Only those diagnostic categories with at least one occurrence appear in this table.

**Standardized to age distribution of 2000 U.S. population. 
Idaho National Laboratory 2010

OSHA Data

Appendix L. Number of Workers with at Least One OSHA Event by Gender, Age, and Job Category*

\begin{tabular}{|c|c|c|c|c|c|c|c|c|c|c|c|}
\hline \multirow{3}{*}{ Job Category } & \multicolumn{5}{|c|}{ Women } & \multicolumn{5}{|c|}{ Men } & \multirow[b]{3}{*}{ TOTAL } \\
\hline & \multicolumn{4}{|c|}{ Age Group } & \multirow[b]{2}{*}{ TOTAL } & \multicolumn{4}{|c|}{ Age Group } & \multirow[b]{2}{*}{ TOTAL } & \\
\hline & $16-29$ & $30-39$ & $40-49$ & $50+$ & & $16-29$ & $30-39$ & $40-49$ & $50+$ & & \\
\hline Professional & 0 & 0 & 0 & 0 & 0 & 0 & 0 & 2 & 0 & 2 & 2 \\
\hline Administrative Support & 0 & 0 & 0 & 1 & 1 & 0 & 0 & 0 & 0 & 0 & 1 \\
\hline Technical Support & 0 & 0 & 1 & 0 & 1 & 0 & 0 & 0 & 0 & 0 & 1 \\
\hline Service & 0 & 0 & 1 & 0 & 1 & 0 & 0 & 0 & 1 & 1 & 2 \\
\hline Security and Fire & 0 & 0 & 2 & 1 & 3 & 1 & 0 & 2 & 4 & 7 & 10 \\
\hline Crafts & 1 & 1 & 1 & 4 & 7 & 2 & 4 & 5 & 8 & 19 & 26 \\
\hline Line Operators & 1 & 0 & 3 & 0 & 4 & 1 & 2 & 0 & 7 & 10 & 14 \\
\hline TOTAL & 2 & 1 & 8 & 6 & 17 & 4 & 6 & 9 & 20 & 39 & 56 \\
\hline
\end{tabular}

*Only those job categories and gender/age combinations with at least one OSHA event appear in this table.

Appendix M. Total Number of Workdays Lost or with Restricted Activity from OSHA Events by Gender and Age

\begin{tabular}{|c|c|c|c|c|c|c|c|c|c|}
\hline \multirow[b]{2}{*}{ Age Group } & \multicolumn{3}{|c|}{ Women } & \multicolumn{3}{|c|}{ Men } & \multicolumn{3}{|c|}{ TOTAL } \\
\hline & $\begin{array}{l}\text { Number of } \\
\text { Events }\end{array}$ & $\begin{array}{c}\text { Days } \\
\text { Restricted }\end{array}$ & Days Lost & $\begin{array}{l}\text { Number of } \\
\text { Events }\end{array}$ & $\begin{array}{c}\text { Days } \\
\text { Restricted }\end{array}$ & Days Lost & $\begin{array}{l}\text { Number of } \\
\text { Events }\end{array}$ & $\begin{array}{c}\text { Days } \\
\text { Restricted }\end{array}$ & Days Lost \\
\hline $16-29$ & 2 & 0 & 6 & 4 & 125 & 13 & 6 & 125 & 19 \\
\hline $30-39$ & 1 & 0 & 0 & 6 & 64 & 63 & 7 & 64 & 63 \\
\hline $40-49$ & 8 & 132 & 275 & 9 & 171 & 88 & 17 & 303 & 363 \\
\hline $50+$ & 6 & 2 & 66 & 20 & 406 & 379 & 26 & 408 & 445 \\
\hline TOTAL & 17 & 134 & 347 & 39 & 766 & 543 & 56 & 900 & 890 \\
\hline
\end{tabular}

Appendix N. Total Number of Workdays Lost or with Restricted Activity from OSHA Events by Gender and Job Category*

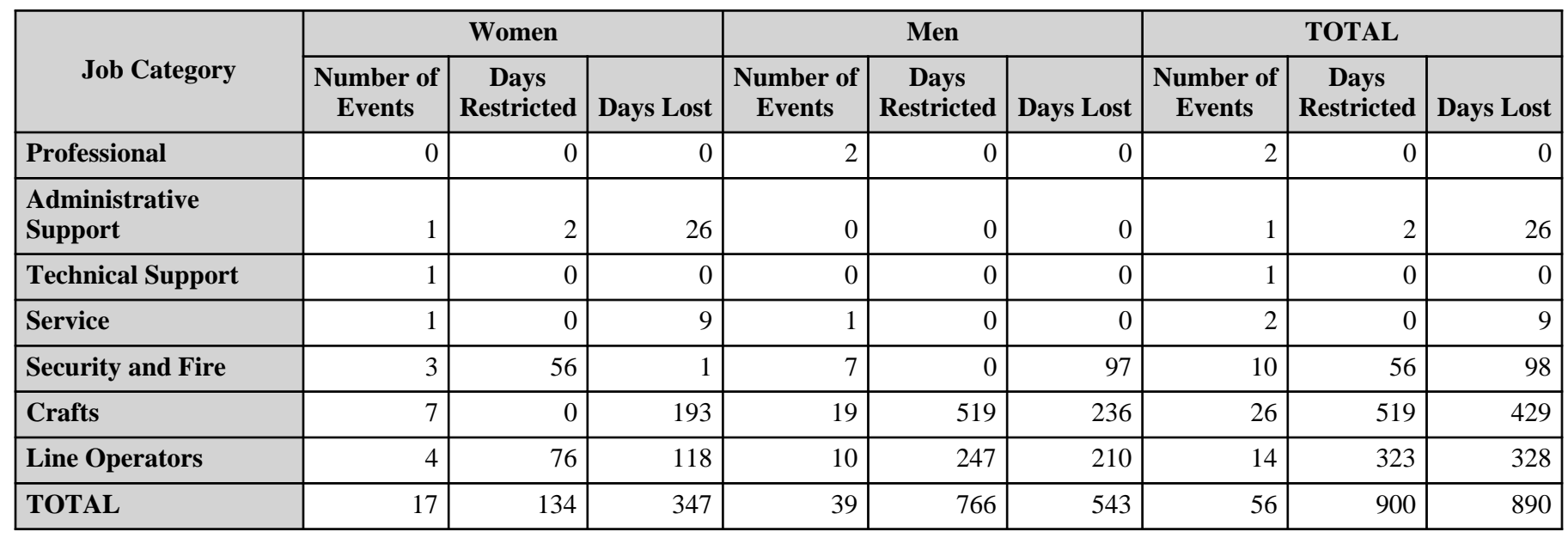

*Only those job categories with at least one OSHA event appear in this table. 
Idaho National Laboratory 2010

OSHA Data

Appendix O. Number of Diagnoses in Each Diagnostic Category by Gender and Age*

\begin{tabular}{|c|c|c|c|c|c|c|}
\hline & & \multicolumn{5}{|c|}{ Women } \\
\hline & & \multicolumn{4}{|c|}{ Age Group } & \multirow[b]{2}{*}{ TOTAL } \\
\hline & & $16-29$ & 30 - 39 & $40-49$ & $50+$ & \\
\hline Diagnostic Category & ICD-9-CM code & \multirow[b]{2}{*}{0} & \multirow[b]{2}{*}{0} & \multirow[b]{2}{*}{2} & \multirow[b]{2}{*}{0} & \multirow[b]{2}{*}{2} \\
\hline NERVOUS SYSTEM (NS) \& SENSE ORGANS & 320-389 & & & & & \\
\hline -Disorders of Peripheral NS & $350-359$ & 0 & 0 & 2 & 0 & 2 \\
\hline -Disorders of Eye & $360-379$ & 0 & 0 & 0 & 0 & 0 \\
\hline CIRCULATORY SYSTEM & $390-459$ & 0 & 0 & 0 & 0 & 0 \\
\hline -Ischemic Heart Dis & $410-414$ & 0 & 0 & 0 & 0 & 0 \\
\hline DIGESTIVE SYSTEM & $520-579$ & 0 & 0 & 0 & 0 & 0 \\
\hline -Oral Cavity, Saliva Glands, Jaw & $520-529$ & 0 & 0 & 0 & 0 & 0 \\
\hline -Hernias & $550-553$ & 0 & 0 & 0 & 0 & 0 \\
\hline $\begin{array}{l}\text { MUSCULOSKELETAL \& CONNECTIVE } \\
\text { TISSUE }\end{array}$ & 710-739 & 3 & 1 & 9 & 5 & 18 \\
\hline -Arthropathies & 710-719 & 2 & 1 & 6 & 2 & 11 \\
\hline -Dorsopathies & $720-724$ & 1 & 0 & 3 & 3 & 7 \\
\hline -Rheumatism, Excluding Back & 725-729 & 0 & 0 & 0 & 0 & 0 \\
\hline $\begin{array}{l}\text { SYMPTOMS, SIGNS, \& ILL-DEFINED } \\
\text { CONDITIONS }\end{array}$ & 780-799 & 1 & 0 & 2 & 0 & 3 \\
\hline -Symptoms & 780-789 & 1 & 0 & 2 & 0 & 3 \\
\hline INJURY \& POISONING & 800-999 & 3 & 1 & 8 & 8 & 20 \\
\hline -Fracture - Upper Limb & 810-819 & 0 & 0 & 0 & 1 & 1 \\
\hline -Fracture - Lower Limb & $820-829$ & 0 & 0 & 0 & 0 & 0 \\
\hline -Dislocation & 830-839 & 0 & 0 & 0 & 0 & 0 \\
\hline -Sprains \& Strains - Back & 846-847 & 1 & 0 & 2 & 4 & 7 \\
\hline -Sprains \& Strains - Other & $840-845,848$ & 0 & 1 & 5 & 2 & 8 \\
\hline -Open Wound - Head, Neck, Trunk & 870-879 & 0 & 0 & 0 & 1 & 1 \\
\hline -Open Wound - Upper Limb & $880-887$ & 0 & 0 & 1 & 0 & 1 \\
\hline -Open Wound - Lower Limb & 890-897 & 0 & 0 & 0 & 0 & 0 \\
\hline -Superficial Injury & 910-919 & 0 & 0 & 0 & 0 & 0 \\
\hline -Contusion & 920-924 & 0 & 0 & 0 & 0 & 0 \\
\hline -Foreign Body Entering Orifice & 930-939 & 0 & 0 & 0 & 0 & 0 \\
\hline -Complications \& Unspecified Injuries & 958-959 & 2 & 0 & 0 & 0 & 2 \\
\hline
\end{tabular}

*Only those diagnostic categories and gender/age combinations with at least one OSHA event appear in this table. 
Idaho National Laboratory 2010

OSHA Data

Appendix O. Number of Diagnoses in Each Diagnostic Category by Gender and Age*

\begin{tabular}{|c|c|c|c|c|c|c|c|}
\hline & & \multicolumn{5}{|c|}{ Men } & \multirow[b]{3}{*}{ TOTAL } \\
\hline & & \multicolumn{4}{|c|}{ Age Group } & \multirow[b]{2}{*}{ TOTAL } & \\
\hline & & $16-29$ & 30 - 39 & $40-49$ & $\mathbf{5 0 +}$ & & \\
\hline Diagnostic Category & ICD-9-CM code & & & & & & \\
\hline NERVOUS SYSTEM (NS) \& SENSE ORGANS & $320-389$ & 1 & 0 & 0 & 1 & 2 & 4 \\
\hline -Disorders of Peripheral NS & $350-359$ & 0 & 0 & 0 & 0 & 0 & 2 \\
\hline -Disorders of Eye & $360-379$ & 1 & 0 & 0 & 1 & 2 & 2 \\
\hline CIRCULATORY SYSTEM & $390-459$ & 0 & 0 & 0 & 1 & 1 & 1 \\
\hline -Ischemic Heart Dis & $410-414$ & 0 & 0 & 0 & 1 & 1 & 1 \\
\hline DIGESTIVE SYSTEM & $520-579$ & 0 & 1 & 0 & 1 & 2 & 2 \\
\hline -Oral Cavity, Saliva Glands, Jaw & $520-529$ & 0 & 1 & 0 & 0 & 1 & 1 \\
\hline -Hernias & $550-553$ & 0 & 0 & 0 & 1 & 1 & 1 \\
\hline $\begin{array}{l}\text { MUSCULOSKELETAL \& CONNECTIVE } \\
\text { TISSUE }\end{array}$ & 710-739 & 2 & 8 & 10 & 14 & 34 & 52 \\
\hline -Arthropathies & $710-719$ & 1 & 1 & 9 & 9 & 20 & 31 \\
\hline -Dorsopathies & $720-724$ & 1 & 4 & 1 & 3 & 9 & 16 \\
\hline -Rheumatism, Excluding Back & 725-729 & 0 & 3 & 0 & 2 & 5 & 5 \\
\hline $\begin{array}{l}\text { SYMPTOMS, SIGNS, \& ILL-DEFINED } \\
\text { CONDITIONS }\end{array}$ & 780-799 & 0 & 1 & 0 & 3 & 4 & 7 \\
\hline -Symptoms & 780-789 & 0 & 1 & 0 & 3 & 4 & 7 \\
\hline INJURY \& POISONING & $800-999$ & 6 & 8 & 10 & 21 & 45 & 65 \\
\hline -Fracture - Upper Limb & 810-819 & 0 & 0 & 0 & 2 & 2 & 3 \\
\hline -Fracture - Lower Limb & $820-829$ & 0 & 1 & 0 & 1 & 2 & 2 \\
\hline -Dislocation & 830-839 & 1 & 1 & 1 & 1 & 4 & 4 \\
\hline -Sprains \& Strains - Back & 846-847 & 1 & 1 & 1 & 2 & 5 & 12 \\
\hline -Sprains \& Strains - Other & $840-845,848$ & 0 & 2 & 6 & 5 & 13 & 21 \\
\hline -Open Wound - Head, Neck, Trunk & $870-879$ & 0 & 0 & 0 & 2 & 2 & 3 \\
\hline -Open Wound - Upper Limb & \begin{tabular}{|l|}
$880-887$ \\
\end{tabular} & 1 & 2 & 1 & 4 & 8 & 9 \\
\hline -Open Wound - Lower Limb & 890-897 & 0 & 0 & 0 & 1 & 1 & 1 \\
\hline -Superficial Injury & 910-919 & 1 & 0 & 0 & 1 & 2 & 2 \\
\hline -Contusion & $920-924$ & 1 & 1 & 1 & 1 & 4 & 4 \\
\hline -Foreign Body Entering Orifice & 930-939 & 1 & 0 & 0 & 0 & 1 & 1 \\
\hline -Complications \& Unspecified Injuries & \begin{tabular}{|c|}
$958-959$ \\
\end{tabular} & 0 & 0 & 0 & 1 & 1 & 3 \\
\hline
\end{tabular}

\begin{tabular}{|c|c|c|c|c|c|c|c|c|c|c|c|}
\hline & \multicolumn{5}{|c|}{ Women } & \multicolumn{5}{|c|}{ Men } & \multirow[b]{3}{*}{ TOTAL } \\
\hline & \multicolumn{4}{|c|}{ Age Group } & \multirow[b]{2}{*}{ TOTAL } & \multicolumn{4}{|c|}{ Age Group } & \multirow[b]{2}{*}{ TOTAL } & \\
\hline & $16-29$ & 30 - 39 & $40-49$ & $50+$ & & $16-29$ & 30 - 39 & $40-49$ & $50+$ & & \\
\hline Diagnostic Category & \multirow[b]{2}{*}{7} & \multirow[b]{2}{*}{2} & \multirow[b]{2}{*}{21} & \multirow[b]{2}{*}{13} & \multirow[b]{2}{*}{43} & \multirow[b]{2}{*}{9} & \multirow[b]{2}{*}{18} & \multirow[b]{2}{*}{20} & \multirow[b]{2}{*}{41} & \multirow[b]{2}{*}{88} & \\
\hline Total & & & & & & & & & & & 131 \\
\hline
\end{tabular}

*Only those diagnostic categories and gender/age combinations with at least one OSHA event appear in this table. 
Idaho National Laboratory 2010

OSHA Data

Appendix P. Number of Workdays Lost or with Restricted Activity in Each Diagnostic Category by Gender and Age*

\begin{tabular}{|c|c|c|c|c|c|c|c|c|c|}
\hline & & \multicolumn{8}{|c|}{ Women } \\
\hline & & \multicolumn{8}{|c|}{ Age Group } \\
\hline & & \multicolumn{2}{|c|}{$16-29$} & \multicolumn{2}{|c|}{30 - 39} & \multicolumn{2}{|c|}{$40-49$} & \multicolumn{2}{|c|}{$50+$} \\
\hline & & $\begin{array}{c}\text { Days } \\
\text { Restricted }\end{array}$ & $\begin{array}{l}\text { Days } \\
\text { Lost }\end{array}$ & $\begin{array}{c}\text { Days } \\
\text { Restricted }\end{array}$ & $\begin{array}{l}\text { Days } \\
\text { Lost }\end{array}$ & \begin{tabular}{|c|} 
Days \\
Restricted
\end{tabular} & $\begin{array}{l}\text { Days } \\
\text { Lost }\end{array}$ & $\begin{array}{c}\text { Days } \\
\text { Restricted }\end{array}$ & $\begin{array}{l}\text { Days } \\
\text { Lost }\end{array}$ \\
\hline Diagnostic Category & ICD-9-CM Codes & & & & & & & & \\
\hline -Disorders of Peripheral NS & $350-359$ & 0 & 0 & 0 & 0 & 76 & 89 & 0 & 0 \\
\hline -Arthropathies & 710-719 & 0 & 0 & 0 & 0 & 76 & 274 & 0 & 27 \\
\hline -Dorsopathies & $720-724$ & 0 & 6 & 0 & 0 & 0 & 157 & 2 & 60 \\
\hline -Symptoms & 780-789 & 0 & 0 & 0 & 0 & 56 & 54 & 0 & 0 \\
\hline -Fracture - Upper Limb & $810-819$ & 0 & 0 & 0 & 0 & 0 & 0 & 0 & 5 \\
\hline -Sprains \& Strains - Back & 846-847 & 0 & 6 & 0 & 0 & 0 & 148 & 2 & 61 \\
\hline -Sprains \& Strains - Other & $840-845,848$ & 0 & 0 & 0 & 0 & 56 & 185 & 0 & 27 \\
\hline -Open Wound - Head, Neck, Trunk & $870-879$ & 0 & 0 & 0 & 0 & 0 & 0 & 0 & 1 \\
\hline -Open Wound - Upper Limb & $880-887$ & 0 & 0 & 0 & 0 & 0 & 0 & 0 & 0 \\
\hline $\begin{array}{l}\text {-Complications \& Unspecified } \\
\text { Injuries }\end{array}$ & 958-959 & 0 & 0 & 0 & 0 & 0 & 0 & 0 & 0 \\
\hline
\end{tabular}

\begin{tabular}{|c|c|c|c|c|c|c|c|c|c|}
\hline & & \multicolumn{8}{|c|}{ Men } \\
\hline & & \multicolumn{8}{|c|}{ Age Group } \\
\hline & & \multicolumn{2}{|c|}{$16-29$} & \multicolumn{2}{|c|}{$30-39$} & \multicolumn{2}{|c|}{$40-49$} & \multicolumn{2}{|c|}{$50+$} \\
\hline & & $\begin{array}{c}\text { Days } \\
\text { Restricted }\end{array}$ & $\begin{array}{l}\text { Days } \\
\text { Lost }\end{array}$ & $\begin{array}{c}\text { Days } \\
\text { Restricted }\end{array}$ & $\begin{array}{l}\text { Days } \\
\text { Lost }\end{array}$ & $\begin{array}{c}\text { Days } \\
\text { Restricted }\end{array}$ & $\begin{array}{l}\text { Days } \\
\text { Lost }\end{array}$ & $\begin{array}{c}\text { Days } \\
\text { Restricted }\end{array}$ & $\begin{array}{l}\text { Days } \\
\text { Lost }\end{array}$ \\
\hline Diagnostic Category & ICD-9-CM Codes & & & & & & & & \\
\hline -Disorders of Eye & $360-379$ & 0 & 0 & 0 & 0 & 0 & 0 & 161 & 19 \\
\hline -Ischemic Heart Dis & $410-414$ & 0 & 0 & 0 & 0 & 0 & 0 & 161 & 19 \\
\hline -Oral Cavity, Saliva Glands, Jaw & $520-529$ & 0 & 0 & 0 & 0 & 0 & 0 & 0 & 0 \\
\hline -Hernias & $550-553$ & 0 & 0 & 0 & 0 & 0 & 0 & 0 & 26 \\
\hline -Arthropathies & $710-719$ & 125 & 13 & 46 & 56 & 170 & 76 & 296 & 312 \\
\hline -Dorsopathies & $720-724$ & 0 & 0 & 0 & 0 & 0 & 0 & 0 & 0 \\
\hline -Rheumatism, Excluding Back & $725-729$ & 0 & 0 & 46 & 56 & 0 & 0 & 254 & 93 \\
\hline -Symptoms & 780-789 & 0 & 0 & 0 & 0 & 0 & 0 & 161 & 19 \\
\hline -Fracture - Upper Limb & $810-819$ & 0 & 0 & 0 & 0 & 0 & 0 & 0 & 0 \\
\hline -Fracture - Lower Limb & $820-829$ & 0 & 0 & 0 & 0 & 0 & 0 & 107 & 0 \\
\hline -Dislocation & $830-839$ & 125 & 13 & 0 & 0 & 79 & 37 & 0 & 41 \\
\hline -Sprains \& Strains - Back & 846-847 & 0 & 0 & 0 & 0 & 0 & 0 & 0 & 0 \\
\hline -Sprains \& Strains - Other & $840-845,848$ & 0 & 0 & 46 & 56 & 171 & 88 & 134 & 213 \\
\hline -Open Wound - Head, Neck, Trunk & 870-879 & 0 & 0 & 0 & 0 & 0 & 0 & 0 & 0 \\
\hline -Open Wound - Upper Limb & $880-887$ & 0 & 0 & 18 & 7 & 0 & 0 & 3 & 0 \\
\hline -Open Wound - Lower Limb & 890-897 & 0 & 0 & 0 & 0 & 0 & 0 & 0 & 0 \\
\hline -Superficial Injury & 910-919 & 0 & 0 & 0 & 0 & 0 & 0 & 0 & 0 \\
\hline
\end{tabular}

(Continued)

*OSHA events with >1 ICD-9-CM code in the same diagnostic category were counted only once. Only those diagnostic categories and gender/age combinations with at least one occurrence appear in this table. 
Idaho National Laboratory 2010

OSHA Data

Appendix P. Number of Workdays Lost or with Restricted Activity in Each Diagnostic Category by Gender and Age*

\begin{tabular}{|c|c|c|c|c|c|c|c|c|c|}
\hline & & \multicolumn{8}{|c|}{ Men } \\
\hline & & \multicolumn{8}{|c|}{ Age Group } \\
\hline & & \multicolumn{2}{|c|}{$16-29$} & \multicolumn{2}{|c|}{30 - 39} & \multicolumn{2}{|c|}{$40-49$} & \multicolumn{2}{|c|}{$50+$} \\
\hline & & \begin{tabular}{|c|} 
Days \\
Restricted
\end{tabular} & $\begin{array}{l}\text { Days } \\
\text { Lost }\end{array}$ & $\begin{array}{c}\text { Days } \\
\text { Restricted }\end{array}$ & $\begin{array}{l}\text { Days } \\
\text { Lost }\end{array}$ & $\begin{array}{c}\text { Days } \\
\text { Restricted }\end{array}$ & $\begin{array}{l}\text { Days } \\
\text { Lost }\end{array}$ & $\begin{array}{c}\text { Days } \\
\text { Restricted }\end{array}$ & $\begin{array}{c}\text { Days } \\
\text { Lost }\end{array}$ \\
\hline Diagnostic Category & ICD-9-CM Codes & & & & & & & & \\
\hline -Contusion & $920-924$ & 0 & 0 & 0 & 0 & 0 & 0 & 1 & 80 \\
\hline -Foreign Body Entering Orifice & 930-939 & 0 & 0 & 0 & 0 & 0 & 0 & 0 & 0 \\
\hline $\begin{array}{l}\text {-Complications \& Unspecified } \\
\text { Injuries }\end{array}$ & 958-959 & 0 & 0 & 0 & 0 & 0 & 0 & 1 & 80 \\
\hline
\end{tabular}

*OSHA events with >1 ICD-9-CM code in the same diagnostic category were counted only once. Only those diagnostic categories and gender/age combinations with at least one occurrence appear in this table. 
Idaho National Laboratory 2010

OSHA Data

Appendix Q. Number of Occurrences in Each Accident Category by Gender and Age*

\begin{tabular}{|c|c|c|c|c|c|c|}
\hline & & \multicolumn{5}{|c|}{ Women } \\
\hline & & \multicolumn{4}{|c|}{ Age Group } & \multirow[b]{2}{*}{ TOTAL } \\
\hline & & $16-29$ & 30 - 39 & $40-49$ & $\mathbf{5 0 +}$ & \\
\hline Type of Accident & E Codes & & & & & \\
\hline Motor Vehicle Traffic & E810-E819 & 0 & 0 & 0 & 0 & 0 \\
\hline Motor Vehicle Nontraffic & E820-E825 & 0 & 0 & 0 & 0 & 0 \\
\hline Falls & E880-E888 & 0 & 0 & 0 & 2 & 2 \\
\hline $\begin{array}{l}\text { Submersion/Suffocation/Foreign } \\
\text { Bodies }\end{array}$ & E910-E915 & 0 & 0 & 0 & 0 & 0 \\
\hline Other Accidents & E916-E928 & 2 & 1 & 8 & 4 & 15 \\
\hline
\end{tabular}

\begin{tabular}{|c|c|c|c|c|c|c|c|}
\hline & & \multicolumn{5}{|c|}{ Men } & \multirow[b]{3}{*}{ TOTAL } \\
\hline & & \multicolumn{4}{|c|}{ Age Group } & \multirow[b]{2}{*}{ ГOTAL } & \\
\hline & & $16-29$ & 30 - 39 & $40-49$ & $50+$ & & \\
\hline Type of Accident & E Codes & & & & & & \\
\hline Motor Vehicle Traffic & E810-E819 & 1 & 0 & 0 & 1 & 2 & 2 \\
\hline Motor Vehicle Nontraffic & E820-E825 & 0 & 0 & 0 & 1 & 1 & 1 \\
\hline Falls & E880-E888 & 0 & 1 & 3 & 5 & 9 & 11 \\
\hline $\begin{array}{l}\text { Submersion/Suffocation/Foreign } \\
\text { Bodies }\end{array}$ & E910-E915 & 1 & 0 & 0 & 0 & 1 & 1 \\
\hline Other Accidents & E916-E928 & 2 & 5 & 6 & 13 & 26 & 41 \\
\hline
\end{tabular}

*Only those accident types and gender/age combinations with at least one occurrence appear in this table. 
Idaho National Laboratory 2010

OSHA Data

Appendix R. Number of Workdays Lost or with Restricted Activity in Each Accident Category by Gender and Age*

\begin{tabular}{|c|c|c|c|c|c|c|c|c|c|}
\hline & & \multicolumn{8}{|c|}{ Women } \\
\hline & & \multicolumn{8}{|c|}{ Age Group } \\
\hline & & \multicolumn{2}{|c|}{$16-29$} & \multicolumn{2}{|c|}{30 - 39} & \multicolumn{2}{|c|}{$40-49$} & \multicolumn{2}{|c|}{$50+$} \\
\hline & & $\begin{array}{c}\text { Days } \\
\text { Restricted }\end{array}$ & $\begin{array}{l}\text { Days } \\
\text { Lost }\end{array}$ & $\begin{array}{c}\text { Days } \\
\text { Restricted }\end{array}$ & $\begin{array}{l}\text { Days } \\
\text { Lost }\end{array}$ & $\begin{array}{c}\text { Days } \\
\text { Restricted }\end{array}$ & $\begin{array}{l}\text { Days } \\
\text { Lost }\end{array}$ & $\begin{array}{c}\text { Days } \\
\text { Restricted }\end{array}$ & $\begin{array}{l}\text { Days } \\
\text { Lost }\end{array}$ \\
\hline Type of Accident & E Codes & \multirow[b]{2}{*}{0} & \multirow[b]{2}{*}{0} & \multirow[b]{2}{*}{0} & \multirow[b]{2}{*}{0} & \multirow[b]{2}{*}{0} & \multirow[b]{2}{*}{0} & \multirow[b]{2}{*}{0} & \multirow[b]{2}{*}{6} \\
\hline Falls & \begin{tabular}{|l|} 
E880-E888 \\
\end{tabular} & & & & & & & & \\
\hline Other Accidents & \begin{tabular}{|l|} 
E916-E928 \\
\end{tabular} & 0 & 6 & 0 & 0 & 132 & 275 & 2 & 60 \\
\hline
\end{tabular}

\begin{tabular}{|c|c|c|c|c|c|c|c|c|c|}
\hline & & \multicolumn{8}{|c|}{ Men } \\
\hline & & \multicolumn{8}{|c|}{ Age Group } \\
\hline & & \multicolumn{2}{|c|}{$16-29$} & \multicolumn{2}{|c|}{$30-39$} & \multicolumn{2}{|c|}{$40-49$} & \multicolumn{2}{|c|}{$50+$} \\
\hline & & $\begin{array}{c}\text { Days } \\
\text { Restricted }\end{array}$ & $\begin{array}{l}\text { Days } \\
\text { Lost }\end{array}$ & $\begin{array}{c}\text { Days } \\
\text { Restricted }\end{array}$ & $\begin{array}{l}\text { Days } \\
\text { Lost }\end{array}$ & $\begin{array}{c}\text { Days } \\
\text { Restricted }\end{array}$ & $\begin{array}{l}\text { Days } \\
\text { Lost }\end{array}$ & $\begin{array}{c}\text { Days } \\
\text { Restricted }\end{array}$ & $\begin{array}{l}\text { Days } \\
\text { Lost }\end{array}$ \\
\hline Type of Accident & E Codes & \multirow[b]{2}{*}{0} & \multirow[b]{2}{*}{0} & \multirow[b]{2}{*}{0} & \multirow[b]{2}{*}{0} & \multirow[b]{2}{*}{0} & \multirow[b]{2}{*}{0} & \multirow[b]{2}{*}{0} & \multirow[b]{2}{*}{0} \\
\hline Motor Vehicle Traffic & E810-E819 & & & & & & & & \\
\hline Motor Vehicle Nontraffic & E820-E825 & 0 & 0 & 0 & 0 & 0 & 0 & 0 & 28 \\
\hline Falls & E880-E888 & 0 & 0 & 0 & 0 & 79 & 58 & 201 & 195 \\
\hline $\begin{array}{l}\text { Submersion/Suffocation/Foreign } \\
\text { Bodies }\end{array}$ & E910-E915 & 0 & 0 & 0 & 0 & 0 & 0 & 0 & 0 \\
\hline Other Accidents & E916-E928 & 125 & 13 & 64 & 63 & 92 & 30 & 205 & 156 \\
\hline
\end{tabular}

*OSHA events with $>1$ E code in the same accident type were counted only once. Only those accident types and gender/age combinations with at least one occurrence appear in this table. 
Idaho National Laboratory 2010

OSHA Data

Appendix S. Number of Diagnoses in Each Diagnostic Category by Gender and Job Category*

\begin{tabular}{|c|c|c|c|c|c|c|c|c|}
\hline & & \multicolumn{7}{|c|}{ Women } \\
\hline & & \multicolumn{6}{|c|}{ Job Category } & \multirow[b]{2}{*}{ TOTAL } \\
\hline & & $\begin{array}{c}\text { Administrative } \\
\text { Support }\end{array}$ & $\begin{array}{l}\text { Technical } \\
\text { Support }\end{array}$ & Service & $\begin{array}{l}\text { Security and } \\
\text { Fire }\end{array}$ & Crafts & $\begin{array}{c}\text { Line } \\
\text { Operators }\end{array}$ & \\
\hline Diagnostic Category & ICD-9-CM Code & & & & & & & \\
\hline $\begin{array}{l}\text { NERVOUS SYSTEM } \\
\text { (NS) \& SENSE ORGANS }\end{array}$ & $320-389$ & 0 & 0 & 0 & 0 & 0 & 2 & 2 \\
\hline $\begin{array}{l}\text {-Disorders of Peripheral } \\
\text { NS }\end{array}$ & $350-359$ & 0 & 0 & 0 & 0 & 0 & 2 & 2 \\
\hline $\begin{array}{l}\text { MUSCULOSKELETAL } \\
\text { \& CONNECTIVE } \\
\text { TISSUE }\end{array}$ & 710-739 & 1 & 0 & 2 & 2 & 7 & 6 & 18 \\
\hline -Arthropathies & $710-719$ & 0 & 0 & 1 & 1 & 3 & 6 & 11 \\
\hline -Dorsopathies & $720-724$ & 1 & 0 & 1 & 1 & 4 & 0 & 7 \\
\hline $\begin{array}{l}\text { SYMPTOMS, } \\
\text { SIGNS, \& ILL-DEFINED } \\
\text { CONDITIONS }\end{array}$ & 780-799 & 0 & 0 & 0 & 1 & 0 & 2 & 3 \\
\hline -Symptoms & $780-789$ & 0 & 0 & 0 & 1 & 0 & 2 & 3 \\
\hline INJURY \& POISONING & \begin{tabular}{|l|}
$800-999$ \\
\end{tabular} & 1 & 1 & 1 & 3 & 11 & 3 & 20 \\
\hline -Fracture - Upper Limb & $810-819$ & 0 & 0 & 0 & 0 & 1 & 0 & 1 \\
\hline -Sprains \& Strains - Back & 846-847 & 1 & 0 & 0 & 1 & 5 & 0 & 7 \\
\hline $\begin{array}{l}\text {-Sprains \& Strains - } \\
\text { Other }\end{array}$ & $840-845,848$ & 0 & 0 & 1 & 2 & 4 & 1 & 8 \\
\hline $\begin{array}{l}\text {-Open Wound - Head, } \\
\text { Neck, Trunk }\end{array}$ & 870-879 & 0 & 0 & 0 & 0 & 1 & 0 & 1 \\
\hline $\begin{array}{l}\text {-Open Wound - Upper } \\
\text { Limb }\end{array}$ & $880-887$ & 0 & 1 & 0 & 0 & 0 & 0 & 1 \\
\hline $\begin{array}{l}\text {-Complications \& } \\
\text { Unspecified Injuries }\end{array}$ & 958-959 & 0 & 0 & 0 & 0 & 0 & 2 & 2 \\
\hline
\end{tabular}

\begin{tabular}{|c|c|c|c|c|c|c|c|}
\hline & \multicolumn{7}{|c|}{ Women } \\
\hline & \multicolumn{6}{|c|}{ Job Category } & \multirow[b]{2}{*}{ TOTAL } \\
\hline & $\begin{array}{c}\text { Administrative } \\
\text { Support }\end{array}$ & Technical Support & Service & Security and Fire & Crafts & Line Operators & \\
\hline Diagnostic Category & \multirow[b]{2}{*}{2} & \multirow[b]{2}{*}{1} & \multirow[b]{2}{*}{3} & \multirow[b]{2}{*}{6} & \multirow[b]{2}{*}{18} & \multirow[b]{2}{*}{13} & \multirow[b]{2}{*}{43} \\
\hline Total & & & & & & & \\
\hline
\end{tabular}

*Only those diagnostic categories and gender/job category combinations with at least one occurrence appear in this table. 
Idaho National Laboratory 2010

OSHA Data

Appendix S. Number of Diagnoses in Each Diagnostic Category by Gender and Job Category*

\begin{tabular}{|c|c|c|c|c|c|c|c|}
\hline & & \multicolumn{6}{|c|}{ Men } \\
\hline & & \multicolumn{5}{|c|}{ Job Category } & \multirow[b]{2}{*}{ TOTAL } \\
\hline & & Professional & Service & Security and Fire & Crafts & Line Operators & \\
\hline Diagnostic Category & ICD-9-CM Code & & & & & & \\
\hline $\begin{array}{l}\text { NERVOUS SYSTEM } \\
\text { (NS) \& SENSE ORGANS }\end{array}$ & 320-389 & 0 & 0 & 0 & 1 & 1 & 2 \\
\hline -Disorders of Eye & $360-379$ & 0 & 0 & 0 & 1 & 1 & 2 \\
\hline $\begin{array}{l}\text { CIRCULATORY } \\
\text { SYSTEM }\end{array}$ & $390-459$ & 0 & 0 & 0 & 1 & 0 & 1 \\
\hline -Ischemic Heart Dis & $410-414$ & 0 & 0 & 0 & 1 & 0 & 1 \\
\hline DIGESTIVE SYSTEM & $520-579$ & 0 & 0 & 0 & 2 & 0 & 2 \\
\hline $\begin{array}{l}\text {-Oral Cavity, Saliva } \\
\text { Glands, Jaw }\end{array}$ & $520-529$ & 0 & 0 & 0 & 1 & 0 & 1 \\
\hline -Hernias & $550-553$ & 0 & 0 & 0 & 1 & 0 & 1 \\
\hline $\begin{array}{l}\text { MUSCULOSKELETAL } \\
\text { \& CONNECTIVE } \\
\text { TISSUE }\end{array}$ & 710-739 & 3 & 1 & 5 & 18 & 7 & 34 \\
\hline -Arthropathies & $710-719$ & 3 & 0 & 5 & 9 & 3 & 20 \\
\hline -Dorsopathies & $720-724$ & 0 & 1 & 0 & 8 & 0 & 9 \\
\hline $\begin{array}{l}\text {-Rheumatism, Excluding } \\
\text { Back }\end{array}$ & 725-729 & 0 & 0 & 0 & 1 & 4 & 5 \\
\hline $\begin{array}{l}\text { SYMPTOMS, } \\
\text { SIGNS, \& ILL-DEFINED } \\
\text { CONDITIONS }\end{array}$ & 780-799 & 0 & 0 & 1 & 3 & 0 & 4 \\
\hline -Symptoms & 780-789 & 0 & 0 & 1 & 3 & 0 & 4 \\
\hline INJURY \& POISONING & 800-999 & 2 & 2 & 9 & 19 & 13 & 45 \\
\hline -Fracture - Upper Limb & 810-819 & 0 & 0 & 1 & 1 & 0 & 2 \\
\hline -Fracture - Lower Limb & $820-829$ & 0 & 0 & 0 & 0 & 2 & 2 \\
\hline -Dislocation & 830-839 & 0 & 0 & 0 & 4 & 0 & 4 \\
\hline -Sprains \& Strains - Back & 846-847 & 0 & 1 & 0 & 4 & 0 & 5 \\
\hline $\begin{array}{l}\text {-Sprains \& Strains - } \\
\text { Other }\end{array}$ & $840-845,848$ & 2 & 0 & 4 & 4 & 3 & 13 \\
\hline $\begin{array}{l}\text {-Open Wound - Head, } \\
\text { Neck, Trunk }\end{array}$ & 870-879 & 0 & 0 & 0 & 0 & 2 & 2 \\
\hline $\begin{array}{l}\text {-Open Wound - Upper } \\
\text { Limb }\end{array}$ & 880-887 & 0 & 0 & 2 & 4 & 2 & 8 \\
\hline $\begin{array}{l}\text {-Open Wound - Lower } \\
\text { Limb }\end{array}$ & 890-897 & 0 & 1 & 0 & 0 & 0 & 1 \\
\hline -Superficial Injury & 910-919 & 0 & 0 & 1 & 0 & 1 & 2 \\
\hline -Contusion & $920-924$ & 0 & 0 & 1 & 2 & 1 & 4 \\
\hline $\begin{array}{l}\text {-Foreign Body Entering } \\
\text { Orifice }\end{array}$ & 930-939 & 0 & 0 & 0 & 0 & 1 & 1 \\
\hline $\begin{array}{l}\text {-Complications \& } \\
\text { Unspecified Injuries }\end{array}$ & 958-959 & 0 & 0 & 0 & 0 & 1 & 1 \\
\hline
\end{tabular}

*Only those diagnostic categories and gender/job category combinations with at least one occurrence appear in this table. 
Idaho National Laboratory 2010

OSHA Data

Appendix S. Number of Diagnoses in Each Diagnostic Category by Gender and Job Category*

\begin{tabular}{|c|c|c|c|c|c|c|}
\hline & \multicolumn{6}{|c|}{ Men } \\
\hline & \multicolumn{5}{|c|}{ Job Category } & \multirow[b]{2}{*}{ TOTAL } \\
\hline & Professional & Service & Security and Fire & Crafts & Line Operators & \\
\hline Diagnostic Category & \multirow[b]{2}{*}{5} & \multirow[b]{2}{*}{3} & \multirow[b]{2}{*}{15} & \multirow[b]{2}{*}{44} & \multirow[b]{2}{*}{21} & \multirow[b]{2}{*}{88} \\
\hline Total & & & & & & \\
\hline
\end{tabular}

*Only those diagnostic categories and gender/job category combinations with at least one occurrence appear in this table. 
Idaho National Laboratory 2010

OSHA Data

Appendix T. Number of Workdays Lost or with Restricted Activity in Each Diagnostic Category by Gender and Job Category*

\begin{tabular}{|c|c|c|c|c|c|c|c|}
\hline & & \multicolumn{6}{|c|}{ Women } \\
\hline & & \multicolumn{6}{|c|}{ Job Category } \\
\hline & & \multicolumn{2}{|c|}{$\begin{array}{l}\text { Administrative } \\
\text { Support }\end{array}$} & \multicolumn{2}{|c|}{ Technical Support } & \multicolumn{2}{|c|}{ Service } \\
\hline & & \begin{tabular}{c|} 
Days \\
Restricted
\end{tabular} & Days Lost & \begin{tabular}{|c|} 
Days \\
Restricted
\end{tabular} & Days Lost & \begin{tabular}{|c} 
Days \\
Restricted
\end{tabular} & Days Lost \\
\hline Diagnostic Category & ICD-9-CM Codes & \multirow[b]{2}{*}{0} & \multirow[b]{2}{*}{0} & \multirow[b]{2}{*}{0} & \multirow[b]{2}{*}{0} & \multirow[b]{2}{*}{0} & \multirow[b]{2}{*}{0} \\
\hline -Disorders of Peripheral NS & $350-359$ & & & & & & \\
\hline -Arthropathies & $710-719$ & 0 & 0 & 0 & 0 & 0 & 9 \\
\hline -Dorsopathies & $720-724$ & 2 & 26 & 0 & 0 & 0 & 9 \\
\hline -Symptoms & $780-789$ & 0 & 0 & 0 & 0 & 0 & 0 \\
\hline -Fracture - Upper Limb & $810-819$ & 0 & 0 & 0 & 0 & 0 & 0 \\
\hline -Sprains \& Strains - Back & 846-847 & 2 & 26 & 0 & 0 & 0 & 0 \\
\hline -Sprains \& Strains - Other & $840-845,848$ & 0 & 0 & 0 & 0 & 0 & 9 \\
\hline -Open Wound - Head, Neck, Trunk & $870-879$ & 0 & 0 & 0 & 0 & 0 & 0 \\
\hline -Open Wound - Upper Limb & $880-887$ & 0 & 0 & 0 & 0 & 0 & 0 \\
\hline $\begin{array}{l}\text {-Complications \& Unspecified } \\
\text { Injuries }\end{array}$ & 958-959 & 0 & 0 & 0 & 0 & 0 & 0 \\
\hline
\end{tabular}

\begin{tabular}{|c|c|c|c|c|c|c|c|}
\hline & & \multicolumn{6}{|c|}{ Women } \\
\hline & & \multicolumn{6}{|c|}{ Job Category } \\
\hline & & \multicolumn{2}{|c|}{ Security and Fire } & \multicolumn{2}{|c|}{ Crafts } & \multicolumn{2}{|c|}{ Line Operators } \\
\hline & & $\begin{array}{c}\text { Days } \\
\text { Restricted }\end{array}$ & Days Lost & \begin{tabular}{c|} 
Days \\
Restricted
\end{tabular} & Days Lost & \begin{tabular}{c|} 
Days \\
Restricted
\end{tabular} & Days Lost \\
\hline Diagnostic Category & ICD-9-CM Codes & & & & & & \\
\hline -Disorders of Peripheral NS & $350-359$ & 0 & 0 & 0 & 0 & 76 & 89 \\
\hline -Arthropathies & $710-719$ & 0 & 0 & 0 & 174 & 76 & 118 \\
\hline -Dorsopathies & $720-724$ & 0 & 1 & 0 & 187 & 0 & 0 \\
\hline -Symptoms & $780-789$ & 56 & 0 & 0 & 0 & 0 & 54 \\
\hline -Fracture - Upper Limb & $810-819$ & 0 & 0 & 0 & 5 & 0 & 0 \\
\hline -Sprains \& Strains - Back & 846-847 & 0 & 1 & 0 & 188 & 0 & 0 \\
\hline -Sprains \& Strains - Other & $840-845,848$ & 56 & 0 & 0 & 174 & 0 & 29 \\
\hline -Open Wound - Head, Neck, Trunk & $870-879$ & 0 & 0 & 0 & 1 & 0 & $\overline{0}$ \\
\hline -Open Wound - Upper Limb & $880-887$ & 0 & 0 & 0 & 0 & 0 & 0 \\
\hline $\begin{array}{l}\text {-Complications \& Unspecified } \\
\text { Injuries }\end{array}$ & 958-959 & 0 & 0 & 0 & 0 & 0 & 0 \\
\hline
\end{tabular}

*OSHA events with >1 ICD-9-CM code in the same diagnostic category were counted only once. Only those diagnostic categories and gender/job category combinations with at least one occurrence appear in this table. 
Idaho National Laboratory 2010

OSHA Data

Appendix T. Number of Workdays Lost or with Restricted Activity in Each Diagnostic Category by Gender and Job Category*

\begin{tabular}{|c|c|c|c|c|c|c|c|}
\hline & & \multicolumn{6}{|c|}{ Men } \\
\hline & & \multicolumn{6}{|c|}{ Job Category } \\
\hline & & \multicolumn{2}{|c|}{ Professional } & \multicolumn{2}{|c|}{ Service } & \multicolumn{2}{|c|}{ Security and Fire } \\
\hline & & $\begin{array}{c}\text { Days } \\
\text { Restricted }\end{array}$ & Days Lost & \begin{tabular}{c|} 
Days \\
Restricted
\end{tabular} & Days Lost & \begin{tabular}{c|c} 
Days \\
Restricted
\end{tabular} & Days Lost \\
\hline Diagnostic Category & ICD-9-CM Codes & \multirow[b]{2}{*}{0} & \multirow[b]{2}{*}{0} & \multirow[b]{2}{*}{0} & \multirow[b]{2}{*}{0} & \multirow[b]{2}{*}{0} & \multirow[b]{2}{*}{0} \\
\hline -Disorders of Eye & $360-379$ & & & & & & \\
\hline -Ischemic Heart Dis & $410-414$ & 0 & 0 & 0 & 0 & 0 & 0 \\
\hline -Oral Cavity, Saliva Glands, Jaw & $520-529$ & 0 & 0 & 0 & 0 & 0 & 0 \\
\hline -Hernias & $550-553$ & 0 & 0 & 0 & 0 & 0 & 0 \\
\hline -Arthropathies & $710-719$ & 0 & 0 & 0 & 0 & 0 & 97 \\
\hline -Dorsopathies & $720-724$ & 0 & 0 & 0 & 0 & 0 & 0 \\
\hline -Rheumatism, Excluding Back & $725-729$ & 0 & 0 & 0 & 0 & 0 & 0 \\
\hline -Symptoms & 780-789 & 0 & 0 & 0 & 0 & 0 & 0 \\
\hline -Fracture - Upper Limb & $810-819$ & 0 & 0 & 0 & 0 & 0 & 0 \\
\hline -Fracture - Lower Limb & $820-829$ & 0 & 0 & 0 & 0 & 0 & 0 \\
\hline -Dislocation & $830-839$ & 0 & 0 & 0 & 0 & 0 & 0 \\
\hline -Sprains \& Strains - Back & $846-847$ & 0 & 0 & 0 & 0 & 0 & 0 \\
\hline -Sprains \& Strains - Other & $840-845,848$ & 0 & 0 & 0 & 0 & 0 & 97 \\
\hline -Open Wound - Head, Neck, Trunk & 870-879 & 0 & 0 & 0 & 0 & 0 & 0 \\
\hline -Open Wound - Upper Limb & 880-887 & 0 & 0 & 0 & 0 & 0 & 0 \\
\hline -Open Wound - Lower Limb & 890-897 & 0 & 0 & 0 & 0 & 0 & 0 \\
\hline -Superficial Injury & $910-919$ & 0 & 0 & 0 & 0 & 0 & 0 \\
\hline -Contusion & $920-924$ & 0 & 0 & 0 & 0 & 0 & 0 \\
\hline -Foreign Body Entering Orifice & $930-939$ & 0 & 0 & 0 & 0 & 0 & 0 \\
\hline $\begin{array}{l}\text {-Complications \& Unspecified } \\
\text { Injuries }\end{array}$ & 958-959 & 0 & 0 & 0 & 0 & 0 & 0 \\
\hline
\end{tabular}

*OSHA events with >1 ICD-9-CM code in the same diagnostic category were counted only once. Only those diagnostic categories and gender/job category combinations with at least one occurrence appear in this table. 
Idaho National Laboratory 2010

OSHA Data

Appendix T. Number of Workdays Lost or with Restricted Activity in Each Diagnostic Category by Gender and Job Category*

\begin{tabular}{|c|c|c|c|c|c|}
\hline & & \multicolumn{4}{|c|}{ Men } \\
\hline & & \multicolumn{4}{|c|}{ Job Category } \\
\hline & & \multicolumn{2}{|c|}{ Crafts } & \multicolumn{2}{|c|}{ Line Operators } \\
\hline & & $\begin{array}{c}\text { Days } \\
\text { Restricted }\end{array}$ & Days Lost & \begin{tabular}{|c|} 
Days \\
Restricted
\end{tabular} & Days Lost \\
\hline Diagnostic Category & ICD-9-CM Codes & \multirow[b]{2}{*}{161} & \multirow[b]{2}{*}{19} & \multirow[b]{2}{*}{0} & \multirow[b]{2}{*}{0} \\
\hline -Disorders of Eye & $360-379$ & & & & \\
\hline -Ischemic Heart Dis & $410-414$ & 161 & 19 & 0 & 0 \\
\hline -Oral Cavity, Saliva Glands, Jaw & $520-529$ & 0 & 0 & 0 & 0 \\
\hline -Hernias & $550-553$ & 0 & 26 & 0 & 0 \\
\hline -Arthropathies & $710-719$ & 497 & 150 & 140 & 210 \\
\hline -Dorsopathies & $720-724$ & 0 & 0 & 0 & 0 \\
\hline -Rheumatism, Excluding Back & $725-729$ & 161 & 19 & 139 & 130 \\
\hline -Symptoms & 780-789 & 161 & 19 & 0 & 0 \\
\hline -Fracture - Upper Limb & $810-819$ & 0 & 0 & 0 & 0 \\
\hline -Fracture - Lower Limb & $820-829$ & 0 & 0 & 107 & 0 \\
\hline -Dislocation & $830-839$ & 204 & 91 & 0 & 0 \\
\hline -Sprains \& Strains - Back & 846-847 & 0 & 0 & 0 & 0 \\
\hline -Sprains \& Strains - Other & $840-845,848$ & 212 & 130 & 139 & 130 \\
\hline -Open Wound - Head, Neck, Trunk & 870-879 & 0 & 0 & 0 & 0 \\
\hline -Open Wound - Upper Limb & 880-887 & 21 & 7 & 0 & 0 \\
\hline -Open Wound - Lower Limb & 890-897 & 0 & 0 & 0 & 0 \\
\hline -Superficial Injury & 910-919 & 0 & 0 & 0 & 0 \\
\hline -Contusion & $920-924$ & 0 & 0 & 1 & 80 \\
\hline -Foreign Body Entering Orifice & 930-939 & 0 & 0 & 0 & 0 \\
\hline $\begin{array}{l}\text {-Complications \& Unspecified } \\
\text { Injuries }\end{array}$ & 958-959 & 0 & 0 & 1 & 80 \\
\hline
\end{tabular}

*OSHA events with >1 ICD-9-CM code in the same diagnostic category were counted only once. Only those diagnostic categories and gender/job category combinations with at least one occurrence appear in this table. 
Idaho National Laboratory 2010

OSHA Data

Appendix U. Number of Occurrences in Each Accident Category by Gender and Job Category*

\begin{tabular}{|c|c|c|c|c|c|c|c|c|}
\hline & & \multicolumn{7}{|c|}{ Women } \\
\hline & & \multicolumn{6}{|c|}{ Job Category } & \multirow[b]{2}{*}{ TOTAL } \\
\hline & & $\begin{array}{c}\text { Administrative } \\
\text { Support }\end{array}$ & \begin{tabular}{|c|} 
Technical \\
Support
\end{tabular} & Service & $\begin{array}{c}\text { Security and } \\
\text { Fire }\end{array}$ & Crafts & $\begin{array}{c}\text { Line } \\
\text { Operators }\end{array}$ & \\
\hline Type of Accident & E CODES & \multirow[b]{2}{*}{0} & \multirow[b]{2}{*}{0} & \multirow[b]{2}{*}{0} & \multirow[b]{2}{*}{0} & \multirow[b]{2}{*}{2} & \multirow[b]{2}{*}{0} & \multirow[b]{2}{*}{2} \\
\hline Falls & E880-E888 & & & & & & & \\
\hline Other Accidents & E916-E928 & 1 & 1 & 1 & 3 & 5 & 4 & 15 \\
\hline
\end{tabular}

\begin{tabular}{|c|c|c|c|c|c|c|c|}
\hline & & \multicolumn{6}{|c|}{ Men } \\
\hline & & \multicolumn{5}{|c|}{ Job Category } & \multirow[b]{2}{*}{ TOTAL } \\
\hline & & Professional & Service & $\begin{array}{c}\text { Security and } \\
\text { Fire }\end{array}$ & Crafts & $\begin{array}{c}\text { Line } \\
\text { Operators }\end{array}$ & \\
\hline Type of Accident & E CODES & \multirow[b]{2}{*}{0} & \multirow[b]{2}{*}{1} & \multirow[b]{2}{*}{1} & \multirow[b]{2}{*}{0} & \multirow[b]{2}{*}{0} & \multirow[b]{2}{*}{2} \\
\hline Motor Vehicle Traffic & E810-E819 & & & & & & \\
\hline Motor Vehicle Nontraffic & E820-E825 & 0 & 0 & 1 & 0 & 0 & 1 \\
\hline Falls & E880-E888 & 1 & 0 & 1 & 3 & 4 & 9 \\
\hline $\begin{array}{l}\text { Submersion/Suffocation/Foreign } \\
\text { Bodies }\end{array}$ & E910-E915 & 0 & 0 & 0 & 0 & 1 & 1 \\
\hline Other Accidents & E916-E928 & 1 & 0 & 4 & 16 & 5 & 26 \\
\hline
\end{tabular}

*Only those accident types and gender/job category combinations with at least one occurrence appear in this table. 
Idaho National Laboratory 2010

OSHA Data

Appendix V. Number of Workdays Lost or with Restricted Activity in Each Accident Category by Gender and Job Category*

\begin{tabular}{|c|c|c|c|c|c|c|c|}
\hline & & \multicolumn{6}{|c|}{ Women } \\
\hline & & \multicolumn{6}{|c|}{ Job Category } \\
\hline & & \multicolumn{2}{|c|}{$\begin{array}{l}\text { Administrative } \\
\text { Support }\end{array}$} & \multicolumn{2}{|c|}{ Technical Support } & \multicolumn{2}{|c|}{ Service } \\
\hline & & $\begin{array}{c}\text { Days } \\
\text { Restricted }\end{array}$ & Days Lost & $\begin{array}{c}\text { Days } \\
\text { Restricted }\end{array}$ & Days Lost & $\begin{array}{c}\text { Days } \\
\text { Restricted }\end{array}$ & Days Lost \\
\hline Type of Accident & E Codes & \multirow[b]{2}{*}{0} & \multirow[b]{2}{*}{0} & \multirow[b]{2}{*}{0} & \multirow[b]{2}{*}{0} & \multirow[b]{2}{*}{0} & \multirow[b]{2}{*}{0} \\
\hline Falls & E880-E888 & & & & & & \\
\hline Other Accidents & E916-E928 & 2 & 26 & 0 & 0 & 0 & 9 \\
\hline
\end{tabular}

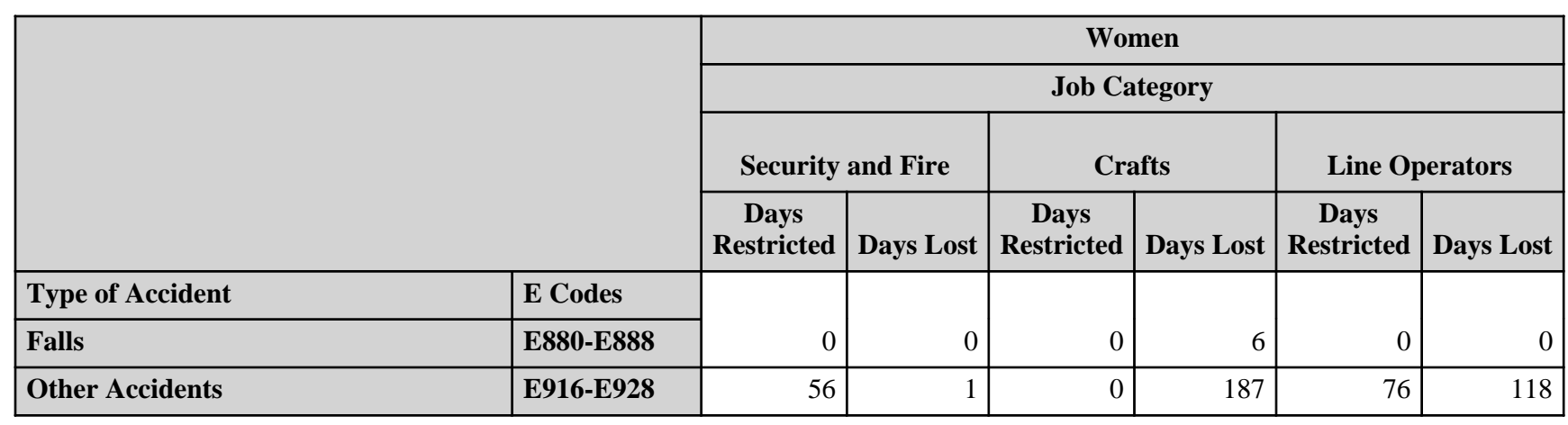

*OSHA events with >1 E code in the same accident type were counted only once. Only those accident types and gender/job category combinations with at least one occurrence appear in this table. 
Idaho National Laboratory 2010

OSHA Data

Appendix V. Number of Workdays Lost or with Restricted Activity in Each Accident Category by Gender and Job Category*

\begin{tabular}{|c|c|c|c|c|c|c|c|}
\hline & & \multicolumn{6}{|c|}{ Men } \\
\hline & & \multicolumn{6}{|c|}{ Job Category } \\
\hline & & \multicolumn{2}{|c|}{ Professional } & \multicolumn{2}{|c|}{ Service } & \multicolumn{2}{|c|}{ Security and Fire } \\
\hline & & $\begin{array}{c}\text { Days } \\
\text { Restricted }\end{array}$ & Days Lost & \begin{tabular}{c|} 
Days \\
Restricted
\end{tabular} & Days Lost & \begin{tabular}{c|} 
Days \\
Restricted
\end{tabular} & Days Lost \\
\hline Type of Accident & E Codes & \multirow[b]{2}{*}{0} & \multirow[b]{2}{*}{0} & \multirow[b]{2}{*}{0} & \multirow[b]{2}{*}{0} & \multirow[b]{2}{*}{0} & \multirow[b]{2}{*}{0} \\
\hline Motor Vehicle Traffic & E810-E819 & & & & & & \\
\hline Motor Vehicle Nontraffic & E820-E825 & 0 & 0 & 0 & 0 & 0 & 28 \\
\hline Falls & E880-E888 & 0 & 0 & 0 & 0 & 0 & 21 \\
\hline $\begin{array}{l}\text { Submersion/Suffocation/Foreign } \\
\text { Bodies }\end{array}$ & E910-E915 & 0 & 0 & 0 & 0 & 0 & 0 \\
\hline Other Accidents & E916-E928 & 0 & 0 & 0 & 0 & 0 & 48 \\
\hline
\end{tabular}

\begin{tabular}{|c|c|c|c|c|c|}
\hline & \multicolumn{4}{|c|}{ Men } \\
\hline & & \multicolumn{4}{|c|}{ Job Category } \\
\hline & & \multicolumn{2}{|c|}{ Crafts } & \multicolumn{2}{|c|}{ Line Operators } \\
\hline & & $\begin{array}{c}\text { Days } \\
\text { Restricted }\end{array}$ & Days Lost & $\begin{array}{c}\text { Days } \\
\text { Restricted }\end{array}$ & Days Lost \\
\hline Type of Accident & E Codes & \multirow[b]{2}{*}{0} & \multirow[b]{2}{*}{0} & \multirow[b]{2}{*}{0} & \multirow[b]{2}{*}{0} \\
\hline Motor Vehicle Traffic & E810-E819 & & & & \\
\hline Motor Vehicle Nontraffic & E820-E825 & 0 & 0 & 0 & 0 \\
\hline Falls & E880-E888 & 79 & 78 & 201 & 154 \\
\hline $\begin{array}{l}\text { Submersion/Suffocation/Foreign } \\
\text { Bodies }\end{array}$ & E910-E915 & 0 & 0 & 0 & 0 \\
\hline Other Accidents & E916-E928 & 440 & 158 & 46 & 56 \\
\hline
\end{tabular}

*OSHA events with >1 E code in the same accident type were counted only once. Only those accident types and gender/job category combinations with at least one occurrence appear in this table. 
Idaho National Laboratory 2010

OSHA Data

Appendix W. Age-Adjusted OSHA Illness and Injury Rates by Diagnostic Category*

Part 1. Men

\begin{tabular}{|c|c|c|c|c|c|}
\hline & & $\begin{array}{l}\text { Number of } \\
\text { Diagnoses }\end{array}$ & $\begin{array}{c}\text { Age-Adjusted } \\
\text { Rate per } 1,000 * *\end{array}$ & $\begin{array}{c}\text { Lower } 95 \% \\
\text { Confidence } \\
\text { Limit per } 1,000\end{array}$ & $\begin{array}{c}\text { Upper } 95 \% \\
\text { Confidence } \\
\text { Limit per 1,000 }\end{array}$ \\
\hline Diagnostic Category & ICD-9-CM Code & \multirow[b]{2}{*}{2} & \multirow[b]{2}{*}{1.2} & \multirow[b]{2}{*}{0.2} & \multirow[b]{2}{*}{6.2} \\
\hline NERVOUS SYSTEM (NS) \& SENSE ORGANS & 320-389 & & & & \\
\hline -Disorders of Eye & $360-379$ & 2 & 1.2 & 0.2 & 6.2 \\
\hline CIRCULATORY SYSTEM & $390-459$ & 1 & 0.2 & 0.0 & 1.5 \\
\hline -Ischemic Heart Dis & $410-414$ & 1 & 0.2 & 0.0 & 1.5 \\
\hline DIGESTIVE SYSTEM & $520-579$ & 2 & 0.6 & 0.1 & 2.7 \\
\hline -Oral Cavity, Saliva Glands, Jaw & $520-529$ & 1 & 0.4 & 0.1 & 2.9 \\
\hline -Hernias & $550-553$ & 1 & 0.2 & 0.0 & 1.5 \\
\hline $\begin{array}{l}\text { MUSCULOSKELETAL \& CONNECTIVE } \\
\text { TISSUE }\end{array}$ & $710-739$ & 34 & 9.4 & 6.2 & 14.3 \\
\hline -Arthropathies & $710-719$ & 20 & 4.6 & 2.6 & 8.1 \\
\hline -Dorsopathies & $720-724$ & 9 & 3.2 & 1.4 & 7.2 \\
\hline -Rheumatism, Excluding Back & 725-729 & 5 & 1.6 & 0.6 & 4.0 \\
\hline $\begin{array}{l}\text { SYMPTOMS, SIGNS, \& ILL-DEFINED } \\
\text { CONDITIONS }\end{array}$ & 780-799 & 4 & 0.9 & 0.3 & 2.8 \\
\hline -Symptoms & $780-789$ & 4 & 0.9 & 0.3 & 2.8 \\
\hline INJURY \& POISONING & $800-999$ & 45 & 14.3 & 9.7 & 21.2 \\
\hline -Fracture - Upper Limb & $810-819$ & 2 & 0.3 & 0.1 & 1.4 \\
\hline -Fracture - Lower Limb & 820-829 & 2 & 0.5 & 0.1 & 2.6 \\
\hline -Dislocation & $830-839$ & 4 & 1.8 & 0.6 & 6.0 \\
\hline -Sprains \& Strains - Back & 846-847 & 5 & 1.9 & 0.6 & 5.9 \\
\hline -Sprains \& Strains - Other & $840-845,848$ & 13 & 2.7 & 1.5 & 5.0 \\
\hline -Open Wound - Head, Neck, Trunk & 870-879 & 2 & 0.3 & 0.1 & 1.4 \\
\hline -Open Wound - Upper Limb & $880-887$ & 8 & 2.6 & 1.0 & 6.4 \\
\hline -Open Wound - Lower Limb & $890-897$ & 1 & 0.1 & 0.0 & $\overline{0.8}$ \\
\hline -Superficial Injury & $910-919$ & 2 & 1.2 & 0.2 & 6.2 \\
\hline -Contusion & 920-924 & 4 & 1.7 & 0.5 & 6.0 \\
\hline -Foreign Body Entering Orifice & 930-939 & 1 & 1.0 & 0.1 & 7.0 \\
\hline -Complications of Surgical/Medical Care & 996-999 & 1 & 0.1 & 0.0 & 0.8 \\
\hline Total & & 88 & 26.7 & 20.4 & 35.0 \\
\hline
\end{tabular}

* Only those diagnostic categories with at least one occurrence appear in this table.

**Standardized to age distribution of 2000 U.S. population. 
Idaho National Laboratory 2010

OSHA Data

Appendix W. Age-Adjusted OSHA IIlness and Injury Rates by Diagnostic Category*

Part 2. Women

\begin{tabular}{|c|c|c|c|c|c|}
\hline & & $\begin{array}{l}\text { Number of } \\
\text { Diagnoses }\end{array}$ & $\begin{array}{c}\text { Age-Adjusted } \\
\text { Rate per } 1,000 * *\end{array}$ & \begin{tabular}{|c|} 
Lower $95 \%$ \\
Confidence \\
Limit per 1,000
\end{tabular} & $\begin{array}{c}\text { Upper } 95 \% \\
\text { Confidence } \\
\text { Limit per 1,000 }\end{array}$ \\
\hline Diagnostic Category & ICD-9-CM Code & \multirow[b]{2}{*}{2} & \multirow[b]{2}{*}{1.2} & \multirow[b]{2}{*}{0.3} & \multirow[b]{2}{*}{4.6} \\
\hline NERVOUS SYSTEM (NS) \& SENSE ORGANS & 320-389 & & & & \\
\hline -Disorders of Peripheral NS & $350-359$ & 2 & 1.2 & 0.3 & 4.6 \\
\hline $\begin{array}{l}\text { MUSCULOSKELETAL \& CONNECTIVE } \\
\text { TISSUE }\end{array}$ & 710-739 & 18 & 15.4 & 8.5 & 27.8 \\
\hline -Arthropathies & $710-719$ & 11 & 10.2 & 4.9 & 21.3 \\
\hline -Dorsopathies & $720-724$ & 7 & 5.2 & 2.0 & 14.0 \\
\hline $\begin{array}{l}\text { SYMPTOMS, SIGNS, \& ILL-DEFINED } \\
\text { CONDITIONS }\end{array}$ & 780-799 & 3 & 3.5 & 0.9 & 13.9 \\
\hline -Symptoms & 780-789 & 3 & 3.5 & 0.9 & 13.9 \\
\hline INJURY \& POISONING & 800-999 & 20 & 16.0 & 9.0 & 28.3 \\
\hline -Fracture - Upper Limb & $810-819$ & 1 & 0.4 & 0.1 & 2.7 \\
\hline -Sprains \& Strains - Back & $846-847$ & 7 & 5.0 & 1.8 & 13.8 \\
\hline -Sprains \& Strains - Other & $840-845,848$ & 8 & 4.9 & 2.3 & 10.5 \\
\hline -Open Wound - Head, Neck, Trunk & 870-879 & 1 & 0.4 & 0.1 & 2.7 \\
\hline -Open Wound - Upper Limb & 880-887 & 1 & 0.6 & 0.1 & 4.1 \\
\hline -Complications of Surgical/Medical Care & 996-999 & 2 & 4.7 & 1.2 & 18.6 \\
\hline Total & & 43 & 36.0 & 24.5 & 52.9 \\
\hline
\end{tabular}

* Only those diagnostic categories with at least one occurrence appear in this table.

**Standardized to age distribution of 2000 U.S. population. 
Idaho National Laboratory 2010

OSHA Data

Appendix W. Age-Adjusted OSHA Illness and Injury Rates by Diagnostic Category*

Part 3. Men and Women

\begin{tabular}{|c|c|c|c|c|c|}
\hline & & $\begin{array}{l}\text { Number of } \\
\text { Diagnoses }\end{array}$ & $\begin{array}{c}\text { Age-Adjusted } \\
\text { Rate per } 1,000 * *\end{array}$ & \begin{tabular}{|c|} 
Lower $95 \%$ \\
Confidence \\
Limit per 1,000
\end{tabular} & $\begin{array}{c}\text { Upper } 95 \% \\
\text { Confidence } \\
\text { Limit per 1,000 }\end{array}$ \\
\hline Diagnostic Category & ICD-9-CM Code & & & & \\
\hline NERVOUS SYSTEM (NS) \& SENSE ORGANS & 320-389 & 4 & 1.2 & 0.3 & 4.0 \\
\hline -Disorders of Peripheral NS & 350-359 & 2 & 0.3 & 0.1 & 1.3 \\
\hline -Disorders of Eye & $360-379$ & 2 & 0.9 & 0.2 & 4.3 \\
\hline CIRCULATORY SYSTEM & $390-459$ & 1 & 0.2 & 0.0 & 1.2 \\
\hline -Ischemic Heart Dis & $410-414$ & 1 & 0.2 & 0.0 & 1.2 \\
\hline DIGESTIVE SYSTEM & $520-579$ & 2 & 0.5 & 0.1 & 2.0 \\
\hline -Oral Cavity, Saliva Glands, Jaw & $520-529$ & 1 & 0.3 & 0.0 & 2.2 \\
\hline -Hernias & $550-553$ & 1 & 0.2 & 0.0 & 1.2 \\
\hline $\begin{array}{l}\text { MUSCULOSKELETAL \& CONNECTIVE } \\
\text { TISSUE }\end{array}$ & 710-739 & 52 & 11.2 & 8.0 & 15.9 \\
\hline -Arthropathies & $710-719$ & 31 & 6.2 & 4.0 & 9.9 \\
\hline -Dorsopathies & $720-724$ & 16 & 3.8 & 2.0 & 7.1 \\
\hline -Rheumatism, Excluding Back & $725-729$ & 5 & 1.2 & 0.5 & 3.1 \\
\hline $\begin{array}{l}\text { SYMPTOMS, SIGNS, \& ILL-DEFINED } \\
\text { CONDITIONS }\end{array}$ & 780-799 & 7 & 1.8 & 0.7 & 4.4 \\
\hline -Symptoms & 780-789 & 7 & 1.8 & 0.7 & 4.4 \\
\hline INJURY \& POISONING & $800-999$ & 65 & 14.9 & 10.8 & 20.5 \\
\hline -Fracture - Upper Limb & 810-819 & 3 & 0.4 & 0.1 & 1.2 \\
\hline -Fracture - Lower Limb & 820-829 & 2 & 0.4 & 0.1 & 2.0 \\
\hline -Dislocation & 830-839 & 4 & 1.3 & 0.4 & 4.2 \\
\hline -Sprains \& Strains - Back & 846-847 & 12 & 2.7 & 1.2 & 5.9 \\
\hline -Sprains \& Strains - Other & $840-845,848$ & 21 & 3.3 & 2.1 & 5.3 \\
\hline -Open Wound - Head, Neck, Trunk & 870-879 & 3 & 0.4 & 0.1 & 1.2 \\
\hline -Open Wound - Upper Limb & 880-887 & 9 & 2.1 & 0.9 & 4.7 \\
\hline -Open Wound - Lower Limb & 890-897 & 1 & 0.1 & 0.0 & 0.7 \\
\hline -Superficial Injury & 910-919 & 2 & 0.9 & 0.2 & 4.3 \\
\hline -Contusion & $920-924$ & 4 & 1.2 & 0.4 & 4.2 \\
\hline -Foreign Body Entering Orifice & 930-939 & 1 & 0.7 & 0.1 & 4.8 \\
\hline -Complications of Surgical/Medical Care & 996-999 & 3 & 1.5 & 0.4 & 5.4 \\
\hline Total & & 131 & 29.7 & 23.8 & 37.1 \\
\hline
\end{tabular}

* Only those diagnostic categories with at least one occurrence appear in this table.

**Standardized to age distribution of 2000 U.S. population. 